TRANSACTIONS OF THE

AMERICAN MATHEMATICAL SOCIETY

Volume 349, Number 1, January 1997, Pages 373-411

S 0002-9947(97)01701-7

\title{
DECOMPOSITION OF BIRATIONAL TORIC MAPS IN BLOW-UPS AND BLOW-DOWNS
}

\author{
JAROSŁAW WŁODARCZYK
}

\begin{abstract}
We prove that a toric birational map between two complete smooth toric varieties of the same dimension can be decomposed in a sequence of equivariant blow-ups and blow-downs along smooth centers.
\end{abstract}

\section{INTRODUCTION}

Two questions, concerning factorization of birational morphisms and maps by blow-ups along smooth varieties, are of fundamental importance in birational algebraic geometry.

Let $X^{\prime}$ and $X^{\prime \prime}$ be complete algebraic varieties which are birationally equivalent.

(1) Does there exist a third variety $X$ and birational morphisms $X \longrightarrow X^{\prime}, X \longrightarrow$ $X^{\prime \prime}$, which are compositions of blow-ups along closed irreducible subvarieties?

(2) Does there exist a sequence of varieties $X_{i}$, for $i=0, \ldots, n$, such that $X_{0}=$ $X^{\prime}, X_{n}=X^{\prime \prime}$, and $X_{i+1}$ is obtained from $X_{i}$ by a blow-up or blow-down along a closed irreducible subvariety?

So far an affirmative answer to (1) was given for the surface case by Italian mathematicians (see for instance [3]). In higher dimensions nothing in general is known, except the famous Hironaka's theorem on elimination of points of indeterminacy, which states that for any pair $X^{\prime}, X^{\prime \prime}$ of birationally equivalent complete complex algebraic varieties there is a finite sequence of blow-ups in smooth closed irreducible subvarieties $X \longrightarrow X^{\prime}$, such that there exists a birational morphism $g: X \longrightarrow X^{\prime \prime}[5]$.

The present work gives a positive answer to (2) in the case of smooth toric varieties. The toric version of question (2) appears in [7] and [8], and is called the weak Oda conjecture. So far the conjecture has been solved by Danilov in the case when the dimension is $\leq 3$ [2]. There is a theorem due to M.Reid in [9], closely related to the above problem, which shows a way of breaking down a birational toric morphism of varieties with mild singularities into elementary steps. The elementary steps, however, are more complicated than blow-ups and blow-downs.

Toric varieties have proven their value as a testing ground for investigating birational morphisms and maps. (See for instance [6], [9].) The behaviour of toric varieties permits us to understand, at least partially, the general picture of arbitrary algebraic varieties. On the other hand, the combinatorial theorems obtained here

Received by the editors March 9, 1994 and, in revised form, December 15, 1995.

1991 Mathematics Subject Classification. Primary 14E05; Secondary 14L32.

This work was partially supported by Deutsche Forschungsgemeischaft and KBN.

(C)1997 American Mathematical Society 
can be directly applied to a larger class of objects, such as toroidal embeddings [6] or spherical spaces.

The present paper is divided into 3 parts. In the preliminaries we introduce the main notions of fan theory. Then, using the fan language, we formulate the main combinatorial theorem of the paper (Theorem A). In Part 1 we talk about general properies of complexes. We introduce basic definitions and notation used through the whole paper. In Part 2 we prove the main theorem for simplicial complexes (Theorem 8.1). Then we associate to any simplicial fan a simplicial complex and deduce from Theorem 8.1 the simplicial case of Theorem A. In Part 3 we deal with complexes equipped with some integral structure, corresponding to the lattice structure on the fan. We also complete the proof of Theorem A.

I would like to thank Professors A. Białynicki-Birula J. Jurkiewicz and J. Wiśniewski for advice and help.

After this paper was submitted for publication I learned that a similar result was obtained by Robert Morelli.

\section{PRELIMINARIES}

\section{Fans: Basic Properties and Notations. Formulation of the Main Theorem in the Fan Language}

We recall briefly the notation from the theory of fans. Fix the rational vector space $\mathbf{Q}^{n}$, containing the lattice $\mathbf{Z}^{n}$.

For any vectors $\left(v_{i}\right), i=1, \ldots, k$, in $\mathbf{Q}^{n}$ by $\left\langle v_{1}, \ldots, v_{k}\right\rangle$ we shall denote the cone spanned by these vectors.

By a fan we shall understand the finite set $\Sigma$ of convex cones in $\mathbf{Q}^{n}$ provided:

1. Every cone $\delta \in \Sigma$ can be written in the form $\delta=\left\langle e_{1}, \ldots e_{k}\right\rangle$, where $e_{i} \in \mathbf{Z}^{n} \subset$ $\mathbf{Q}^{n}$.

2. Every face $\tau$ of a cone $\delta \in \Sigma$ belongs to $\Sigma$.

3. Cones are adjacent along their faces.

4. $\{0\} \in \Sigma$.

A fan $\Sigma$ is regular iff every cone $\delta \in \Sigma$ can be represented in the form $\delta=$ $\left\langle e_{1}, \ldots, e_{k}\right\rangle$, where $\left\{e_{i}\right\}$ is a subset of a basis of $\mathbf{Z}^{n}$. A fan is simplicial iff every cone belonging to it is simplicial. A support of a fan $\Sigma$ is the set $|\Sigma|=\bigcup_{\delta \in \Sigma} \delta$. A decomposition of a fan $\Sigma$ is another fan $\Sigma^{\prime}$ such that $|\Sigma|=\left|\Sigma^{\prime}\right|$ and $\forall_{\delta^{\prime} \in \Sigma^{\prime}} \exists_{\delta \in \Sigma^{\prime}} \delta^{\prime} \subset \delta$. A decomposition $\Sigma^{\prime}$ of a fan $\Sigma$ is regular iff it is a decomposition and a regular fan. A fan $\Sigma$ in $\mathbf{Q}^{n}$ is complete if $|\Sigma|=\mathbf{Q}^{n}$.

There is a bijection between the fans in $\mathbf{Q}^{n} \supset \mathbf{Z}^{n}$ and the normal torus embeddings of the $n$-dimensional torus $T$ [8, Theorem 1.4]. In the above correspondence the decompositions are associated with the $T$-equivariant proper morphisms (identical on $T)$ [8, Corollary 1.17]. The regular fans are associated with the smooth torus embeddings [8, Theorem 1.10], and the simplicial fans are in correspondence with the $Q$-factorial embeddings ([9]). The complete fans correspond to the complete torus embeddings [8, Theorem 1.11] (see also [2], [6], [7]).

Let $\Sigma$ be a fan in $\mathbf{Q}^{n}$. Let $v$ be any vector in $\mathbf{Q}^{n}$. By the blow-up at the ray $\langle v\rangle$ we mean the decomposition which changes every cone $\delta$ of $\Sigma$ containing $\langle v\rangle$ to the set of the convex hulls $\sigma+v$, where $\sigma$ is a face of $\delta$ which does not contain $\langle v\rangle$. The inverse transformation will be called a blow-down. We shall say that a blow-up is regular iff both fans, i.e. before the blow-up and after the blow-up, are regular. The inverse transformation of a regular blow-up is called a regular blow-down. 
Assume that $\Sigma$ is a regular fan. One can show that a blow-up at the primitive vector $v$ is regular iff vector $v$ can be written in the form $v=b_{1}+\ldots+b_{k}$ for some cone $\delta \in \Sigma$ such that $\delta=\left\langle b_{1}, \ldots, b_{k}\right\rangle$, where $b_{i}, i=1, \ldots, k$, is a subset of a basis of $\mathbf{Z}^{n}$ [8, Proposition 1.26].

In the above mentioned correspondence blow-ups are associated with some blowups along $T$-equivariant subshemes (which can be non-reduced). The regular blowups are in $1-1$ correspondence with the blow-ups along closed $T$-equivariant submanifolds.

The main theorem of this paper is the following:

Theorem A. Let $\Sigma^{\prime}, \Sigma^{\prime \prime}$ be two fans in $\mathbf{Q}^{n}$, such that $\left|\Sigma^{\prime}\right|=\left|\Sigma^{\prime \prime}\right|$. Assume that $\Sigma^{\prime}, \Sigma^{\prime \prime}$ " are regular (resp. simplicial). Then there is a sequence of regular (resp. simplicial) fans $\Sigma_{i}$ for $i=0, \ldots, n$, such that $\Sigma_{0}=\Sigma^{\prime}, \Sigma_{n}=\Sigma^{\prime \prime}$ and each $\Sigma_{i}$ is obtained from $\Sigma_{i-1}$ by a blow-up or blow-down for $i=1, \ldots, n$ (which is regular if $\Sigma^{\prime}$ and $\Sigma^{\prime \prime}$ are regular).

We prove this theorem for simplicial fans in Part 2, and for regular fans in Part 3. Via the correspondence between fans and torus embeddings we can deduce from this theorem the following result.

Theorem B. Let $X^{\prime}$ and $X^{\prime \prime}$ be smooth embeddings of torus T. Assume that either

i) $X^{\prime}, X^{\prime \prime}$ are complete

or

ii) there is an equivariant proper morphism $\Phi: X^{\prime} \longrightarrow X^{\prime \prime}$.

Then there exists a sequence of smooth torus embeddings $X_{i}$, for $i=0, \ldots, n$, such that $X_{0}=X^{\prime}, X_{n}=X^{\prime \prime}$, and $X_{i+1}$ is obtained from $X_{i}$ by a T-equivariant blow-up or blow-down along a closed smooth $T$-equivariant irreducible subvariety.

\section{PART I}

\section{Complexes. Basic Properties and Notations}

We will consider in this work geometric objects of the affine space $\mathbf{Q}^{n} \subset \mathbf{R}^{n}$, where $\mathbf{Q}$ is the set of the rational numbers in $\mathbf{R}$.

We adopt all of the definitions and notations from the theory of convex bodies and complexes in affine real space to the case of the field $\mathbf{Q}$. Thus we are going to use the standard notions like simplex, polytope, vertex, face, convex hull (conv), affine hull (aff), relative interior (int), relative boundary ( $\partial$ ), of the polytopes. (For the definitions see [4].)

By $\Delta\left(A_{0}, \ldots, A_{n}\right)$ we shall denote the simplex with vertices $A_{0}, \ldots, A_{n}$. By $P=$ $\operatorname{conv}\left(A_{0}, \ldots, A_{n}\right)$ we mean the polytope which is a convex hull of points $A_{0}, \ldots, A_{n}$. By aff $(X)$ we denote the affine hull of a set $X$. In particular aff $\left(A_{0}, \ldots, A_{n}\right)$ denote the affine hull of the points $A_{0}, \ldots, A_{n}$.

By a complex in $\mathbf{Q}^{d}$, we understand here a finite family $\mathbf{C}$ of polytopes in $\mathbf{Q}^{d}$ such that:

i) every face of member of $\mathbf{C}$ is itself a member of $\mathbf{C}$, and

ii) the intersection of any two members of $\mathbf{C}$ is a face of each of them.

We shall call a member of a complex $\mathbf{C}$ a face of $\mathbf{C}$. A complex $\mathbf{C}$ will be called simplicial provided all its members are simplices. By a subcomplex $\mathbf{C}^{\prime}$ of $\mathbf{C}$ we will understand a subset of $\mathbf{C}$ satisfying property i) from the above definition. By the 
support of a complex $\mathbf{C}$ we mean the set $|\mathbf{C}|=\bigcup_{\mathbf{P} \in \mathbf{C}} \mathbf{P}$. By a subdivision of a complex $\mathbf{C}$ we shall mean another complex $\mathbf{C}^{\prime}$ provided:

i) for every face there exists a face $\rho^{\prime} \in \mathbf{C}^{\prime}$ of $\mathbf{C}$ such that $\rho^{\prime} \subset \rho$ and

ii) $\left|\mathbf{C}^{\prime}\right|=|\mathbf{C}|$.

We shall say that a subdivision $\mathbf{C}^{\prime}$ of $\mathbf{C}$ is proper if the set of vertices of $\mathbf{C}$ equals the set of vertices of $\mathbf{C}^{\prime}: \operatorname{vert}\left(\mathbf{C}^{\prime}\right)=\operatorname{vert}(C)$. By a triangulation of $\mathbf{C}$ we mean a subdivision which is a simplicial complex. By a proper triangulation we shall mean a triangulation which is simultaneously a proper subdivision.

Consider a complex $\mathbf{C}$, its subdivision $\mathbf{S}$, and a subcomplex $\mathbf{L}$ of $\mathbf{C}$. It is clear that $\mathbf{S}$ determines a subdivision of $\mathbf{L}$, which will be called a restriction of $\mathbf{S}$ to $\mathbf{L}$ and denoted by $\mathbf{S}_{\mid \mathbf{L}}$.

We say that a face of a complex is maximal iff it is maximal with respect to the relation of face. For a complex $\mathbf{C}$ denote by $\mathbf{C}_{\max }$ the set of maximal faces of $\mathbf{C}$. Note that $\mathbf{C}_{\max }$ completely determines $\mathbf{C}$.

Let $\mathbf{C}$ be a complex, and $\mathbf{P}$ a face in $\mathbf{C}$. The star of $\mathbf{P}$ in $\mathbf{C}$ is the set $\operatorname{Star}(\mathbf{P} ; \mathbf{C})$, of all faces of $\mathbf{C}$ containing $\mathbf{P}$. We denote by $\operatorname{CompStar}(\mathbf{P} ; \mathbf{C})$ the complex determined by $\operatorname{Star}(\mathbf{P} ; \mathbf{C})$. The antistar $\operatorname{Ast}(\mathbf{P} ; \mathbf{C})$ of $\mathbf{P}$ in $\mathbf{C}$ is the complex consisting of all faces of $\mathbf{C}$ which are disjoint from int $\mathbf{P}$. Note that $\operatorname{Star}(\mathbf{P} ; \mathbf{C}) \cup \operatorname{Ast}(\mathbf{P} ; \mathbf{C})=\mathbf{C}$.

By a complex pair or simply a pair $(\mathbf{C} ; \Omega)$, we understand a pair consisting of a complex $\mathbf{C}$, and a finite subset $\Omega$ of $|\mathbf{C}|$. We will call $\Omega$ the set of added vertices. We define a set of vertices of the pair $(\mathbf{C} ; \Omega)$ as the $\operatorname{set} \operatorname{vert}(\mathbf{C} ; \Omega):=\operatorname{vert} \mathbf{C} \cup \Omega$. By a triangulation (or resp., a subdivision) of the pair $(\mathbf{C} ; \Omega)$ we mean a triangulation $\mathbf{T}$ (resp. subdivision $\mathbf{T}$ ) of the complex $\mathbf{C}$ such that $\operatorname{vert} \mathbf{T}=\operatorname{vert}(\mathbf{C} ; \Omega)$. For example a triangulation of the pair $(\mathbf{C} ; \emptyset)$ is just a proper triangulation.

By a polytope (resp. a simplex) of the pair $(\mathbf{C} ; \Omega$ ) we mean any polytope $\mathbf{P}$ (resp., simplex $\mathbf{P})$ such that $\mathbf{P}$ is contained in some face of $\mathbf{C}$ and $\operatorname{vert} \mathbf{P}=\operatorname{vert}(\mathbf{C} ; \Omega) \cap \mathbf{P}$.

\section{Blow-Ups and Elementary Transformations}

By the blow-up of a complex $\mathbf{K}$ at a point $P \in|\mathbf{K}|$ we mean a subdivision of $\mathbf{K}$ which is obtained from $\mathbf{K}$ by replacing any polytope $\mathbf{P} \in \mathbf{K}$ containing $P$ by the set of convex hulls of $P$ and all faces of $\mathbf{P}$ which do not contain $P$. By the blow-up of a complex $\mathbf{K}$ at a point $P \notin|\mathbf{K}|$ we mean the complex $\mathbf{K}$.

The inverse transformation to a blow-up is called a blow-down.

We denote the blow-up of a complex $\mathbf{K}$ at $P$ by $\mathbf{K}_{(P)}$. Note that if $\mathbf{K}$ is simplicial then $\mathbf{K}_{(P)}$ is also. By $\mathbf{K}_{\left(P_{1} \ldots P_{n}\right)}$ we mean succesive blow-ups of $\mathbf{K}$ at points $P_{1} \ldots, P_{n}$ i.e.

$$
\mathbf{K}_{\left(P_{1} \ldots P_{n}\right)}=\left(\left(\mathbf{K}_{\left(P_{1}\right)}\right) \ldots\right)_{\left(P_{n}\right)}
$$

We call $\mathbf{K}_{\left(P_{1} \ldots P_{n}\right)}$ a blow-up of $\mathbf{K}$ at the sequence of points $P_{1} \ldots, P_{n}$.

Definition 2.1. By a basic polytope $\mathbf{P}^{B}=\operatorname{conv}\left(A_{0}, \ldots, A_{n} ; B_{0}, \ldots, B_{m}\right)$, where $n, m \geq 1$, we mean an $n+m$-dimensional polytope $\operatorname{conv}\left(A_{0}, \ldots, A_{n}, B_{0}, \ldots, B_{m}\right)$, in $\mathbf{Q}^{k}$, such that

$$
\sum_{i=0}^{n} a_{i} A_{j}=\sum_{j=0}^{m} b_{j} B_{j} \quad \text { for some } a_{i}, b_{j}>0 ; \quad \sum_{i=0}^{n} a_{i}=\sum_{j=0}^{m} b_{j}=1 .
$$

By an elementary polytope

$$
\mathbf{P}^{E}=\operatorname{conv}\left(A_{0}, \ldots, A_{n} ; B_{0}, \ldots, B_{m}: C_{1}, \ldots, C_{r}\right)
$$


we mean an $(n+m+r)$-dimensional polytope

$$
\operatorname{conv}\left(A_{0}, \ldots, A_{n}, B_{0}, \ldots, B_{m}, C_{1}, \ldots, C_{r}\right), \quad \text { where } \quad n \geq 1, \quad m \geq 1,
$$

such that:

$$
\sum_{i=0}^{n} a_{i} A_{i}=\sum_{j=0}^{m} b_{j} B_{j} \quad \text { for some } a_{i}, b_{i}>0 ; \quad \sum_{i=0}^{n} a_{i}=\sum_{j=0}^{m} b_{j}=1 .
$$

Example 1.

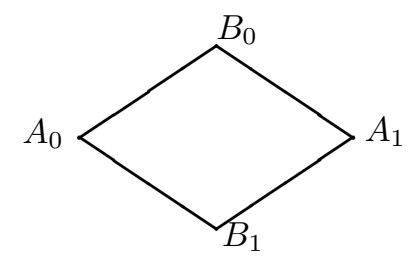

Note that $(1)$ (or $\left(1^{\prime}\right)$ ) is the only relation in the set of vertices of the basic (or elementary) polytope. Let us call each proper triangulation of a basic or an elementary polytope a canonical triangulation.

Lemma 2.2. Each basic polytope $\mathbf{P}^{B}=\operatorname{conv}\left(A_{0}, \ldots, A_{n} ; B_{0}, \ldots, B_{m}\right)$ (or elementary polytope $\left.\mathbf{P}^{E}=\operatorname{conv}\left(A_{0}, \ldots, A_{n} ; B_{0}, \ldots, B_{m}: C_{1}, \ldots, C_{r}\right)\right)$ has exactly two canonical triangulations. These are $\mathbf{A}$ and $\mathbf{B}$, such that

$$
\begin{aligned}
& \mathbf{A}_{\max }=\left\{\Delta\left(A_{0}, \ldots, A_{n}, B_{0}, \ldots, \check{B}_{i}, \ldots, B_{m}\right): \quad i=0, \ldots, m\right\}, \\
& \mathbf{B}_{\max }=\left\{\Delta\left(A_{0}, \ldots, \check{A}_{j}, \ldots, A_{n}, B_{0}, \ldots, B_{m}\right): \quad j=0, \ldots, n\right\},
\end{aligned}
$$

or respectively

$$
\begin{aligned}
& \mathbf{A}_{\max }=\left\{\Delta\left(A_{0}, \ldots, A_{n}, B_{0}, \ldots, \check{B}_{i}, \ldots, B_{m}, C_{1}, \ldots, C_{r}\right)\right\}_{i=0, \ldots, m}, \\
& \mathbf{B}_{\max }=\left\{\Delta\left(A_{0}, \ldots, \check{A}_{j}, \ldots, A_{n} B_{0}, \ldots, B_{m}, C_{1}, \ldots, C_{r}\right)\right\}_{j=0 \ldots, n} .
\end{aligned}
$$

\section{Example 2.}
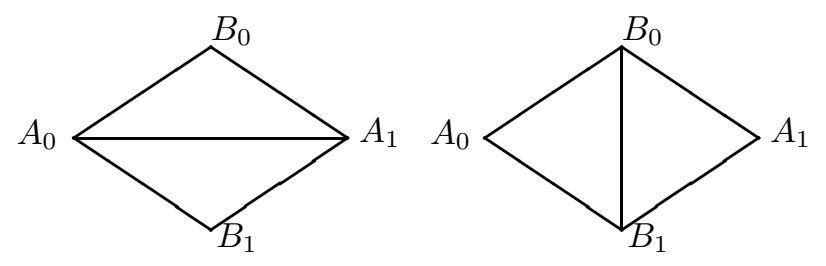

Proof. It suffices to consider the case of a basic polytope. First note that $\mathbf{A}=$ $\mathbf{P}^{B}{ }_{\left(A_{i}\right)}$ for any $A_{i}$, and $\mathbf{B}=\mathbf{P}_{\left(B_{i}\right)}$ for any $B_{j}$. Thus $\mathbf{A}$ and $\mathbf{B}$ are in fact triangulations.

Now note that every $n+m$-dimensional simplex of a proper triangulation is a simplex of $\mathbf{A}_{\max }$ or $\mathbf{B}_{\max }$. On the other hand each simplex from $\mathbf{A}_{\max }$ has the face $\Delta\left(A_{0}, \ldots, A_{n}\right)$, and each simplex from $\mathbf{B}_{\max }$ has the face $\Delta\left(B_{0}, \ldots, B_{m}\right)$. The face $\Delta\left(A_{0}, \ldots, A_{n}\right)$ intersects $\Delta\left(B_{0}, \ldots, B_{m}\right)$ at $P$. Thus there cannot be a triangulation which contain simplices of $\mathbf{A}_{\max }$ and $\mathbf{B}_{\max }$. 
The basic polytope $\mathbf{P}^{B}=\operatorname{conv}\left(A_{0}, \ldots, A_{n} ; B_{0}, \ldots, B_{m}\right)$, as is easily seen, is a face of the elementary polytope $\mathbf{P}^{E}=\operatorname{conv}\left(A_{0}, \ldots, A_{n} ; B_{0}, \ldots, B_{m} ; C_{1}, \ldots, C_{r}\right)$. Triangulations $\mathbf{A}, \mathbf{B}$ of $\mathbf{P}^{B}$ determine triangulations $\mathbf{A}, \mathbf{B}$ of $\mathbf{P}^{E}$.

Definition 2.3. Let $\mathbf{K}$ be a complex.

(a) An elementary subdivision of $\mathbf{K}$ is any subdivision $\mathbf{S}^{E}$, such that:

- there is a basic polytope $\mathbf{P}^{B}$ which is a face of $\mathbf{S}^{E}$,

- every face belonging to $\operatorname{Star}\left(\mathbf{P}^{B} ; \mathbf{S}^{E}\right)$ is an elementary polytope, and

- every face belonging to $\operatorname{Ast}\left(\mathbf{P}^{B} ; \mathbf{S}^{E}\right)$ is a simplex.

(b) The complex $\mathbf{C}^{B}=\operatorname{CompStar}\left(\mathbf{P}^{B} ; \mathbf{S}^{E}\right)$ is called the basic complex of the elementary subdivision.

(c) Any proper triangulation of an elementary subdivision $\mathbf{S}^{E}$ is called a canonical triangulation.

By Lemma 2.2. there are exactly two canonical triangulations of $\mathbf{S}^{E}$, which differ only on $\operatorname{CompStar}\left(\mathbf{P}^{B} ; \mathbf{S}^{E}\right)$, where they are induced by the canonical triangulations of $\mathbf{P}^{B}$.

(d) An elementary transformation of an elementary subdivision changes one of its canonical triangulations to the other. We then say that the two triangulations differ by an elementary transformation.

(e) Two triangulations are elementarily equivalent or el-equivalent iff one of them can be obtained from the other by a sequence of elementary transformations.

From the definitions we deduce that an elementary subdivision determines the two inverse elementary transformations and, conversely, any elementary transformation uniquely determines an elementary subdivision. Thus we will talk about the basic polytopes and canonical triangulations of elementary transformations instead of the basic polytopes and canonical triangulations of elementary subdivisions.

Definition 2.4. Let $\mathbf{K}$ be a complex, and let $\mathbf{T}_{0}, \mathbf{T}_{1}$ denote two triangulations. We call these triangulations equivalent if one of them can be obtained from the other by a sequence of blow-ups and blow-downs.

Proposition 2.5. Let $\mathbf{K}$ be a complex and let $\mathbf{T}_{0}, \mathbf{T}_{1}$ be two triangulations. Assume that $\mathbf{T}_{0}$ and $\mathbf{T}_{1}$ are el-equivalent. Then they are equivalent.

Proof. It suffices to show that any elementary transformation can be obtained by a blow-up and a blow-down.

Let $\mathbf{C}^{B}$ be the basic complex of our elementary transformation. By definition:

$$
\mathbf{C}_{\max }^{B}=\left\{\operatorname{conv}\left(A_{0}, \ldots, A_{n} ; B_{0}, \ldots, B_{m}: C_{1}^{i}, \ldots, C_{m(i)}^{i}\right) ; i \in I\right\} .
$$

Triangulations $\mathbf{T}_{0}$ and $\mathbf{T}_{1}$ coincide outside $\mathbf{C}^{B}$. Their restrictions to $\mathbf{C}^{B}$ are two canonical triangulations $\mathbf{A}, \mathbf{B}$ such that:

$$
\begin{gathered}
\mathbf{A}_{\max }=\left\{\Delta\left(A_{0}, \ldots, A_{n}, B_{0}, \ldots, \check{B}_{j}, \ldots, B_{m}, C_{1}^{i}, \ldots, C_{m(i)}^{i}\right),\right. \\
j=0, \ldots, m, \quad i \in I\}, \\
\mathbf{B}_{\max }=\left\{\begin{array}{c}
\Delta\left(A_{0}, \ldots, \check{A}_{j}, \ldots, A_{n}, B_{0}, \ldots, B_{m}, C_{1}^{i}, \ldots, C_{m(i)}^{i}\right), \\
j=0, \ldots, n, \quad i \in I\} .
\end{array}\right.
\end{gathered}
$$


Assume that $\mathbf{T}_{0}$ on $\mathbf{C}^{B}$ is $\mathbf{A}$, and $\mathbf{T}_{1}$ on $\mathbf{C}^{B}$ is $\mathbf{B}$. Let $P:=\Delta\left(A_{0}, \ldots, A_{n}\right) \cap$ $\Delta\left(B_{0}, \ldots, B_{m}\right)$. Then $\mathbf{T}_{0(P)}$ and $\mathbf{T}_{1(P)}$ coincide outside $\mathbf{C}^{B}$. On $\mathbf{C}^{B}$ both equal

$$
\begin{gathered}
\left\{\Delta\left(A_{0}, \ldots, \check{A}_{r}, \ldots, A_{n}, B_{0}, \ldots, \check{B}_{j}, \ldots, B_{m}, C_{1}^{i}, \ldots, C_{m(i)}^{i}, P\right)\right. \\
j=0, \ldots, m, \quad r=0, \ldots, n, \quad i \in I\} .
\end{gathered}
$$

Consequently we can obtain $\mathbf{T}_{0}$ from $\mathbf{T}_{1}$ by blowing up at $P$, and then blowing down at $P$.

\section{Inducing Triangulations of a Complex By Triangulations of a Subcomplex.}

Let $\mathbf{P}=\operatorname{conv}\left(A_{0}, \ldots, A_{k}, B_{0}, \ldots, B_{m}\right)$ be a polytope such that each $B_{i}$ is affinely independent of $\left\{A_{0}, \ldots, A_{k}, B_{0}, \ldots, B_{m}\right\} \backslash\left\{B_{i}\right\}$. In particular, the polytope $\mathbf{P}^{\prime}=\operatorname{conv}\left(A_{0}, \ldots, A_{k}\right)$ is a face of $\mathbf{P}$. Let $\mathbf{S}$ be a subdivision of $\mathbf{P}^{\prime}$. Then $\mathbf{S}$ determines a subdivision $\mathbf{P}(\mathbf{S})$ of $\mathbf{P}$ such that $\mathbf{P}(\mathbf{S})_{\max }=\left\{\operatorname{conv}\left(\Delta, B_{0}, \ldots, B_{m}\right)\right.$ : $\left.\Delta \in \mathbf{S}_{\max }\right\}$.

We say that a complex $\mathbf{K}$ is simplicial with respect to its subcomplex $\mathbf{R}$ (or simplicial w.r.t. $\mathbf{R}$ ) iff :

a) for every face $\mathbf{k} \in \mathbf{K}, \mathbf{k}^{\prime}=\mathbf{k} \cap|\mathbf{R}|$ is a common face of $\mathbf{k}$ and $\mathbf{R}$, and

b) for every face $\mathbf{k} \in \mathbf{K}$, each vertex $P$ in $\operatorname{vert}(\mathbf{k}) \backslash \operatorname{vert}\left(\mathbf{k}^{\prime}\right)$ is affinely independent of the set vert $(\mathbf{k}) \backslash\{\mathbf{P}\}$.

Examples. A simplicial complex is simplicial w.r.t. any of its faces. Elementary subdivision is simplicial w.r.t. its basic polytope, elementary polytope.

Assume that $\mathbf{C}$ is simplicial w.r.t. its subcomplex $\mathbf{L}$. Then any subdivision $\mathbf{T}$ of $\mathbf{L}$ determines the subdivision $\mathbf{C}(\mathbf{T})$ of $\mathbf{C}$ such that for a polytope $\mathbf{P} \in \mathbf{C}$

$$
\mathbf{C}(\mathbf{T})_{\mid \mathbf{P}}=\mathbf{P}\left(\mathbf{T}_{\mid(|\mathbf{L}| \cap \mathbf{P})}\right) .
$$

The statements below follow easily from the definitions.

Proposition 3.1. If $\mathbf{T}$ is a triangulation of $\mathbf{L}$ then $\mathbf{C}(\mathbf{T})$ is also a triangulation of $\mathbf{C}$ (with the previous assumptions).

Proposition 3.2. Assume that $\mathbf{C}$ is simplicial w.r.t. $\mathbf{L}$. Let $\mathbf{P}$ be a subdivision of $\mathbf{L}$, and $\mathbf{R}$ be a subdivision of $\mathbf{P}$, proper or not. Then $\mathbf{C}(\mathbf{R})$ is a subdivision of $\mathbf{C}(\mathbf{P})$, respectively proper or not.

Proposition 3.3. Assume that $\mathbf{C}$ is simplicial w.r.t. $\mathbf{L}$ and let $\mathbf{R}$ be any subdivision of $\mathbf{L}$. Then $\mathbf{C}(\mathbf{R})$ is simplicial w.r.t. $\mathbf{R}$.

Proposition 3.4. Assume that $\mathbf{C}$ is simplicial w.r.t. $\mathbf{L}$, and let $\mathbf{R}$ be an elementary subdivision of $\mathbf{L}$. Then $\mathbf{C}(\mathbf{R})$ is an elementary subdivision of $\mathbf{C}$.

Corollary 3.5. Let $\mathbf{C}$ be a complex simplicial w.r.t. its subcomplex $\mathbf{L}$. Let $\mathbf{T}_{0}, \mathbf{T}_{1}$ be two triangulations of $\mathbf{L}$.

a) If $\mathbf{T}_{0}, \mathbf{T}_{1}$ differ by an elementary transformation, then $\mathbf{C}\left(\mathbf{T}_{\mathbf{0}}\right), \mathbf{C}\left(\mathbf{T}_{\mathbf{1}}\right)$ do also.

b) If $\mathbf{T}_{0}, \mathbf{T}_{1}$ are el-equivalent, then so are $\mathbf{C}\left(\mathbf{T}_{\mathbf{0}}\right), \mathbf{C}\left(\mathbf{T}_{\mathbf{1}}\right)$.

Proof. Apply Proposition 3.2 to Proposition 3.4.

Proposition 3.6. Assume that $\mathbf{C}$ is simplicial w.r.t. $\mathbf{L}$, and $\mathbf{S}$ is a subdivision of L. Let $W \in|\mathbf{L}|$. Then $\mathbf{C}(\mathbf{S})_{(W)}=\mathbf{C}\left(\mathbf{S}_{(\mathbf{W})}\right)$. 
Proposition 3.7. Let $\mathbf{C}$ be simplicial w.r.t. $\mathbf{L}$. Let $\mathbf{T}_{0}, \mathbf{T}_{1}$ be two equivalent triangulations of $\mathbf{L}$. Then $\mathbf{C}\left(\mathbf{T}_{0}\right)$ and $\mathbf{C}\left(\mathbf{T}_{1}\right)$ are also equivalent.

Proposition 3.8. Let $\mathbf{C}$ be simplicial w.r.t. $\mathbf{L}$. Let $\mathbf{R}_{0}, \mathbf{R}_{1}$ be two subdivisions of $\mathbf{L}$. Then $\mathbf{P}$ is a common polytope of $\mathbf{C}\left(\mathbf{R}_{0}\right)$ and $\mathbf{C}\left(\mathbf{R}_{1}\right)$ iff $\mathbf{P} \cap \mathbf{L}$ is a common polytope of $\mathbf{R}_{0}$ and $\mathbf{R}_{1}$.

\section{PART 2}

\section{El-Equivalence of Some Triangulations}

This section is devoted to the proof of the following proposition:

Proposition 4. A. Let $\Delta$ be a simplex. Let $P, Q \in \Delta$. Assume no proper face of $\Delta$ contains both $P$ and $Q$. Then $\Delta_{(P, Q)}$ and $\Delta_{(Q, P)}$ are el-equivalent, and the elementary transformations realizing the above el-equivalence do not change the triangulations on the relative boundary of $\Delta$.

B. Let $\mathbf{A}, \mathbf{B}$ be the two canonical triangulations of the basic polytope

$$
\mathbf{P}^{\mathbf{B}}=\operatorname{conv}\left(A_{0}, \ldots, A_{n} ; B_{0}, \ldots, B_{m}\right) .
$$

Let $Q \in \mathbf{P}^{\mathbf{B}}$. Then $\mathbf{A}_{(Q)}$ is el-equivalent to $\mathbf{B}_{(Q)}$, and the transformations realizing the el-equivalence do not change the triangulations on the boundary.

C. Let $\mathbf{A}, \mathbf{B}$ be the two canonical triangulations of an elementary polytope $\mathbf{P}^{\mathbf{E}}$. Let $Q \in \mathbf{P}^{\mathbf{E}} \backslash \mathbf{P}^{\mathbf{B}}$. Then $\mathbf{A}_{(Q)}$ is el-equivalent to $\mathbf{B}_{(Q)}$, and the transformations realizing the el-equivalence do not change the triangulations on the boundary.

Lemma 4.0. Let $\mathbf{C}$ be a complex in $\mathbf{Q}^{n}$ and $\mathbf{T}$ be a triangulation. Let $\mathbf{P}^{\mathbf{B}}$ be a basic polytope and let $\left\{\mathbf{P}^{\mathbf{E}}: i \in I\right\}$ be a set of elementary polytopes with the same basic polytope $\mathbf{P}^{\mathbf{B}}$, such that:

1. For each $\mathbf{P}^{\mathbf{E}}{ }_{i}$ one of its canonical triangulations is a subcomplex of $\mathbf{T}$.

2. The triangulation $\mathbf{B}$ of the basic polytope

$$
\mathbf{P}^{\mathbf{B}}=\operatorname{conv}\left(A_{0}, \ldots, A_{n} ; B_{0}, \ldots, B_{m}\right)
$$

is a subcomplex of $\mathbf{T}$. Then $\beta=\Delta\left(B_{0}, \ldots, B_{m}\right)$ is a simplex of $\mathbf{T}$ and for each $\alpha$ in $\operatorname{Star}(\beta ; \mathbf{T})$ there is $\mathbf{P}^{\mathbf{E}}{ }_{i}$ such that $\alpha \subset \mathbf{P}^{\mathbf{E}}{ }_{i}$

Then the set $\mathbf{S}$ of all faces of polytopes in $\operatorname{Ast}(\beta ; \mathbf{T}) \cup\left\{\mathbf{P}_{i}{ }_{i} ; i \in I\right\}$ is an elementary subdivision of $\mathbf{C}$, and $\mathbf{T}$ is one of the canonical triangulations of this subdivision.

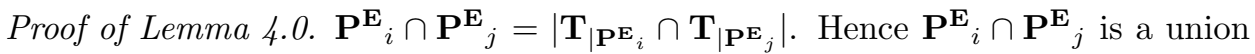
of simplices $\delta \in \mathbf{T}_{\mid P_{i}^{E}} \cap \mathbf{T}_{\mid P_{j}^{E}}$. From the definition of an elementary polytope it follows that if $\delta \in \mathbf{T}_{\mid P_{i}^{E}}$ where $\mathbf{T}$ is a canonical triangulation, then $\operatorname{conv}\left(\delta ; \mathbf{P}^{\mathbf{B}}\right)$ is a face of $\mathbf{P}^{\mathbf{E}}{ }_{i}$. Thus $\mathbf{P}^{\mathbf{E}}{ }_{i} \cap \mathbf{P}^{\mathbf{E}}{ }_{j}$ is a union of common faces $\operatorname{conv}\left(\delta ; \mathbf{P}^{\mathbf{B}}\right)$, and since it is a convex set it must be a single common face of $\mathbf{P}^{\mathbf{E}}{ }_{i}$ and $\mathbf{P}^{\mathbf{E}}{ }_{j}$.

Analogously if $\sigma \in \operatorname{Ast}(\beta ; \mathbf{T})$ then $\mathbf{P}_{i}^{E} \cap \sigma$ is a union of common faces $\delta$ of $\mathbf{T}_{\mid P_{i}^{E}}$ and $\sigma$. Each such $\delta \in \mathbf{T}_{\mid P_{i}^{E}}$ that does not contain $\beta$ is a face of $\mathbf{P}_{i}^{E}$. Thus $\mathbf{P}_{i}^{E} \cap \sigma$ is a union of common faces of $\mathbf{P}_{i}^{E}$ and $\sigma$, and hence by convexity is a single common face.

Finally, any two faces of $\operatorname{Ast}(\beta ; \mathbf{T}) \cup\left\{\mathbf{P}_{i}^{E}: i \in I\right\}$ are adjacent along their faces. Moreover by property 2 we have that $\left|\operatorname{Ast}(\beta ; \mathbf{T}) \cup\left\{\mathbf{P}_{i}^{E}: i \in I\right\}\right|=|\mathbf{C}|$. 
Let $(P ; \Omega)$ be a pair, where $\mathbf{P}$ is a polytope and $\Omega$ is the set of added vertices. Let $V:=\operatorname{vert}(P ; \Omega)$. We can represent any subdivision of this pair by the set of its maximal polytopes. On the other hand each polytope of such a subdivision can be represented as the set of its vertices, which is a subset of $V$. Let $\operatorname{SET}(V)$ denote the set of all subsets of $V$ and $\operatorname{SET}^{2}(V)$ denote the set of all the subsets of $\operatorname{SET}(V)$. Thus each subdivision of the considered pair determines a unique element of $\operatorname{SET}^{2}(V)$.

It follows that we can take set-theoretical unions and differences in $\operatorname{SET}^{2}(V)$. We introduce multiplication in $\operatorname{SET}^{2}(V): \beta_{0} \beta_{1}=\left\{\alpha_{i} \cup \gamma_{j}: \alpha_{i} \in \beta_{0}, \gamma_{j} \in \beta_{1}\right\}$.

Every $\alpha \in \operatorname{SET}(V)$ determines the element $\{\alpha\}$ from $\operatorname{SET}^{2}(V)$. Every nonempty element $\alpha \in \operatorname{SET}(V)$ determines the element $\langle\alpha\rangle \in \operatorname{SET}^{2}(V)$ given by $\langle\alpha\rangle:=$ the set of all $(\operatorname{card}(\alpha)-1)$ subsets of $\alpha$.

In particular we have the following properties.

1. If $\alpha, \beta \in \operatorname{SET}(V)$ and $\alpha \cap \beta=\emptyset$, then $\{\alpha \cup \beta\}=\{\alpha\}\{\beta\}$.

2. If $\alpha, \beta \in \operatorname{SET}(V)$ and $\alpha \cap \beta=\emptyset$, then $\langle\alpha \cup \beta\rangle=\langle\alpha\rangle\{\beta\} \cup\{\alpha\}\langle\beta\rangle$.

3. If $\alpha, \beta, \gamma \in \operatorname{SET}^{2}(V)$, then $\alpha(\beta \cup \gamma)=\alpha \beta \cup \alpha \gamma$.

We shall use these operations and notations in representing triangulations as elements of $\operatorname{SET}^{2}(V)$.

Further, for $\alpha \in \operatorname{SET}(V)$ denote by $\Delta(\alpha)$ and $\operatorname{conv}(\alpha)$ the simplex and the polytope, respectively, spanned by elements belonging to $\alpha$. For $\alpha \in \operatorname{SET}^{2}(V)$, by $\operatorname{conv}(\alpha)$ we mean $\left\{\operatorname{conv}\left(\alpha^{\prime}\right): \alpha^{\prime} \in \alpha\right\}$

Example 1. Let $Q \in \Delta=\Delta\left(A_{0}, \ldots, A_{n}\right)$. Then the triangulation $\Delta_{(Q)}$ is described by $\left\langle\left\{A_{0}, \ldots, A_{n}\right\}\right\rangle\{\{Q\}\}$ in $\operatorname{SET}^{2}(V)$.

Example 2. Let $\mathbf{P}^{\mathbf{B}}=\operatorname{conv}\left(A_{0}, \ldots, A_{n} ; B_{0}, \ldots, B_{m}\right)$ be a basic polytope. Then

$$
\begin{aligned}
& \mathbf{A}=\left\{\left\{A_{0}, \ldots, A_{n}\right\}\right\}\left\langle\left\{B_{0}, \ldots, B_{m}\right\}\right\rangle \in \operatorname{SET}^{2}(V), \\
& \mathbf{B}=\left\langle\left\{A_{0}, \ldots, A_{n}\right\}\right\rangle\left\{\left\{B_{0}, \ldots, B_{m}\right\}\right\} \in \mathrm{SET}^{2}(V) .
\end{aligned}
$$

Proof of Proposition $4 A$. Consider a pair $(\Delta ;\{P, Q\})$, where $P, Q \in \Delta$. Let $\Delta=$ $\Delta\left(A_{0}, \ldots, A_{n}\right)$. Choose the increasing sequence $\left(i_{0}, \ldots, i_{k}\right)$ in $\{0, \ldots, n\}$ such that for all $j=0, \ldots, n$ there are nonnegative $p_{j}, q_{j}$ such that $p_{j} / q_{j} \geq p_{j+1} / q_{j+1}$, with strict inequality for $j=i_{l}$ for some $l=0, \ldots, k$. We put also $i_{-1}=-1$. Since $P$ and $Q$ are not contained in any proper face of $\Delta$, we never have $p_{j}=q_{j}=0$. Hence $q_{j} / p_{j}$ is always a well defined number from $[0,+\infty]$.

Define $\underline{P}:=\{P\}, \underline{Q}:=\{Q\}, \underline{A}_{j}:=\left\{A_{l}: i_{j-1}<l \leq i_{j}\right\} \in \operatorname{SET}(V), j=$ $0, \ldots, k$. Consider 4 cases.

- $(0,0) \quad Q, P \in$ int $\Delta$.

- $(0, P) \quad Q \in$ int $\Delta, P \in \partial \Delta$.

- $(Q, 0) \quad Q \in \partial \Delta, P \in \operatorname{int} \Delta$.

- $(Q, P) \quad Q, P \in \partial \Delta$.

In the cases $(*, 0)$, i.e. $P \in \operatorname{int} \Delta$, we have $\Delta_{(P) \max }=\left\{\Delta\left(A_{0}, \ldots, \check{A}_{i}, \ldots, A_{n}, P\right)\right.$ : $i=0, \ldots, n\}$. (Here $(*, 0)$ means $(0,0)$ and $(Q, 0)$.) Using our new notation we can write

$$
\Delta_{(P)}=\left\langle\underline{A}_{0}, \ldots, \underline{A}_{k}\right\rangle\{P\} \in \operatorname{SET}^{2}(V)
$$

In the cases $(*, P)$, i.e. $P \in \partial \Delta$, we have $q_{0} / p_{0}=\ldots=q_{i_{0}} / p_{i_{0}}=\infty$, and so $P \in \operatorname{int} \Delta\left(\underline{A}_{1} \cup \ldots \cup \underline{A}_{k}\right)$. Thus we have $\Delta_{(P) \max }=\left\{\Delta\left(A_{0}, \ldots, \check{A}_{i}, \ldots, A_{n}, P\right)\right.$ : 
$\left.i=i_{0}+1, \ldots, n\right\}$. We can write it as follows:

$$
\Delta_{(P)}=\left\{\underline{A}_{0}\right\}\left\langle\underline{A}_{1}, \ldots, \underline{A}_{k}\right\rangle\{P\}
$$

or

$$
\Delta_{(P)}=\left\langle\underline{A}_{0}, \ldots, \underline{A}_{k}\right\rangle\{P\} \backslash\left\langle\underline{A}_{0}\right\rangle\left\{\underline{A}_{1} \cup \ldots \cup \underline{A}_{k}\right\} \in \operatorname{SET}^{2}(V) .
$$

Thus in all the cases we have:

$$
\Delta_{(P)}=\left\langle\underline{A}_{0}, \ldots, \underline{A}_{k}\right\rangle\{P\} \backslash \delta^{P}\left\langle\underline{A}_{0}\right\rangle\left\{\underline{A}_{1} \cup \ldots \cup \underline{A}_{k}\right\} \in \operatorname{SET}^{2}(V),
$$

where

$$
\delta^{P}= \begin{cases}0 & \text { in the cases }(*, 0) \\ 1 & \text { in the cases }(*, P)\end{cases}
$$

In the cases $\left(0,{ }^{*}\right)$, i.e. $Q \in \operatorname{int} \Delta$, we have $Q \in \operatorname{int} \Delta\left(\underline{A}_{0} \cup \ldots \underline{A}_{k-1} \cup \underline{P}\right)$. Indeed

$$
Q=\sum_{i=0}^{n} q_{i} A_{i} \text { and } P=\sum_{i=0}^{n} p_{i} A_{i} .
$$

Then

$$
\begin{aligned}
Q & =\sum_{i=0}^{n} q_{i} A_{i}+\left(q_{i_{k}} / p_{i_{k}}\right)\left(P-\sum_{i=0}^{n} p_{i} A_{i}\right) \\
& =\sum_{i=0}^{n}\left(q_{i}-\left(q_{i_{k}} / p_{i_{k}}\right) p_{i}\right) A_{i}+\left(q_{i_{k}} / p_{i_{k}}\right) P .
\end{aligned}
$$

Here $q_{i} \neq 0$ since $Q \in \operatorname{int} \Delta, q_{i} \geq q_{i}-\left(q_{i_{k}} / p_{i_{k}}\right) p_{i} \geq 0$; if $q_{i} / p_{i}=q_{i_{k}} / p_{i_{k}}$ then that coefficient is zero.

In the cases $\left(Q,{ }^{*}\right)$, i.e. $Q \in \partial \Delta$, we have $q_{i_{k-1}+1} / p_{i_{k-1}+1}=\ldots=q_{i_{k}} / p_{i_{k}}=0$ and $Q \in \operatorname{int} \Delta\left(\underline{A}_{0} \cup \ldots \cup \underline{A}_{k-1}\right)$.

The triangulation $\Delta_{(P, Q)}$ is obtained by replacing in $\Delta_{(P)}$ all simplices containing $Q$ by the convex hulls of $Q$ and their faces which do not contain $Q$.

In all the considered cases the set of all maximal simplices of $\Delta_{(P)}$ containing $Q$ is the following:

$$
\left\{\underline{A}_{0} \cup \ldots \cup \underline{A}_{k-1}\right\}\left\langle\underline{A}_{k}\right\rangle\{\underline{P}\} .
$$

In the cases $\left(0,{ }^{*}\right)$ we replace this set by

$$
\left\langle\underline{A}_{0} \cup \ldots \cup \underline{A}_{k-1}\right\rangle\left\langle\underline{A}_{k}\right\rangle\{\underline{P}\}\{\underline{Q}\} \cup\left\{\underline{A}_{0} \cup \ldots \cup \underline{A}_{k-1}\right\}\left\langle\underline{A}_{k}\right\rangle\{\underline{Q}\} .
$$

In the cases $(Q, *)$ we replace it by

$$
\left\langle\underline{A}_{0} \cup \ldots \cup \underline{A}_{k-1}\right\rangle\left\langle\underline{A}_{k}\right\rangle\{\underline{P}\}\{\underline{Q}\} .
$$

By the above

$$
\begin{aligned}
\Delta_{(P, Q)} & =\left\langle\underline{A}_{0} \cup \ldots \cup \underline{A}_{k-1}\right\rangle\left\{\underline{A}_{k}\right\}\{\underline{P}\} \\
& \cup\left\langle\underline{A}_{0} \cup \ldots \cup \underline{A}_{k-1}\right\rangle\left\langle\underline{A}_{k}\right\rangle\{\underline{P}\}\{\underline{Q}\} \\
& \cup\left\{\underline{A}_{0} \cup \ldots \cup \underline{A}_{k-1}\right\}\left\langle\underline{A}_{k}\right\rangle\{\underline{Q}\} \\
& \backslash \delta^{P}\left\langle\underline{A}_{0}\right\rangle\left\{\underline{A}_{1} \cup \ldots \cup \underline{A}_{k}\right\}\{\underline{P}\} \backslash \delta^{Q}\left\{\underline{A}_{0} \cup \ldots \cup \underline{A}_{k-1}\right\}\left\langle\underline{A}_{k}\right\rangle\{\underline{Q}\},
\end{aligned}
$$

where

$$
\delta^{Q}= \begin{cases}0 & \text { in the cases }\left(0,{ }^{*}\right), \\ 1 & \text { in the cases }\left(Q,{ }^{*}\right) .\end{cases}
$$


By symmetry we have

$$
\begin{aligned}
\Delta_{(Q, P)} & =\left\langle\underline{A}_{0}\right\rangle\left\{\underline{A}_{1} \cup \ldots \cup \underline{A}_{k}\right\}\{\underline{P}\} \\
& \cup\left\langle\underline{A}_{0}\right\rangle\left\langle\underline{A}_{1} \cup \ldots \cup \underline{A}_{k}\right\rangle\{\underline{P}\}\{Q\} \\
& \cup\left\{\underline{A}_{0}\right\}\left\langle\underline{A}_{1} \ldots \cup \underline{A}_{k}\right\rangle\{Q\} \\
& \backslash \delta^{P}\left\langle\underline{A}_{0}\right\rangle\left\{\underline{A}_{1} \cup \ldots \cup \underline{A}_{k}\right\}\{\underline{P}\} \backslash \delta^{Q}\left\{\underline{A}_{0} \cup \ldots \cup \underline{A}_{k-1}\right\}\left\langle\underline{A}_{k}\right\rangle\{\underline{Q}\} .
\end{aligned}
$$

Lemma 4.1. For $s=0, \ldots, k-1$ define:

$$
\begin{aligned}
\mathbf{T}_{s}:= & \left\langle\underline{A}_{0} \cup \ldots \cup \underline{A}_{s}\right\rangle\left\{\underline{A}_{s+1} \cup \ldots \cup \underline{A}_{k}\right\}\{\underline{P}\} \\
& \cup\left\langle\underline{A}_{0} \cup \ldots \cup \underline{A}_{s}\right\rangle\left\langle\underline{A}_{s+1} \cup \ldots \cup \underline{A}_{k}\right\rangle\{\underline{P}\}\{\underline{Q}\} \\
& \cup\left\{\underline{A}_{0} \cup \ldots \cup \underline{A}_{s}\right\}\left\langle\underline{A}_{s+1} \cup \ldots \cup \underline{A}_{k}\right\rangle\{\underline{Q}\} \\
& \backslash \delta^{P}\left\langle\underline{A}_{0}\right\rangle\left\{\underline{A}_{1} \cup \ldots \cup \underline{A}_{k}\right\}\{\underline{P}\} \backslash \delta^{Q}\left\{\underline{A}_{0} \cup \ldots \cup \underline{A}_{k-1}\right\}\left\langle\underline{A}_{k}\right\rangle\{\underline{Q}\} .
\end{aligned}
$$

Then $\mathbf{T}_{s}$ is a triangulation; $\mathbf{T}_{0}=\Delta_{(Q, P)}$ and $\mathbf{T}_{k-1}=\Delta_{(P, Q)}$. Moreover $\mathbf{T}_{s}$ differs from $\mathbf{T}_{s+1}$ by an elementary transformation for $s=0, \ldots, k-2$.

Proof. We use induction on $s$.

Assume that $\mathbf{T}_{s}$ is a triangulation. We have to show that $\mathbf{T}_{s+1}$ is a triangulation which differs from $\mathbf{T}_{s}$ by an elementary transformation.

Lemma 4.1.1. For $s=0, \ldots, k-1$ the set

$$
\mathbf{P}^{\mathbf{B}}{ }_{s}:=\operatorname{conv}\left(\underline{A}_{0} \cup \ldots \cup \underline{A}_{s} \cup \underline{P} ; \underline{A}_{s+2} \cup \ldots \cup \underline{A}_{k} \cup \underline{Q}\right)
$$

is a basic polytope.

Proof. Recall that $Q=\sum_{i=0}^{n} q_{i} A_{i}$ and $P=\sum_{i=0}^{n} p_{i} A_{i}$; then

$$
\begin{aligned}
Q & =\sum_{i=0}^{n} q_{i} A_{i}+\left(q_{i_{s+1}} / p_{i_{s+1}}\right)\left(P-\sum_{i=0}^{n} p_{i} A_{i}\right) \\
& =\sum_{i=0}^{n}\left(q_{i}-\left(q_{i_{s+1}} / p_{i_{s+1}}\right) p_{i}\right) A_{i}+\left(q_{i_{s+1}} / p_{i_{s+1}}\right) P .
\end{aligned}
$$

Note that terms in $i_{s+1}$ have coefficient 0 . Hence we have

$$
\begin{aligned}
Q & +\sum_{i=i_{s+1}+1}^{n}\left(q_{i_{s+1}} / p_{i_{s+1}}-q_{i} / p_{i}\right) p_{i} A_{i} \\
& =\left(q_{i_{s+1}} / p_{i_{s+1}}\right) P+\sum_{i=0}^{i_{s}}\left(\left(q_{i} / p_{i}-q_{i_{s+1}} / p_{i_{s+1}}\right) p_{i} A_{i} .\right.
\end{aligned}
$$

All coefficients in the above equality are positive. The sum of the LHS coefficients equals the sum of the RHS coefficients since we subtruct or add the same coefficients in both sides of the first equality. Therefore we can scale by that sum and get the relation in a basic polytope. On the other hand $Q$ is independent of the set $\left(\underline{A}_{0} \cup \ldots \cup \underline{A}_{s-1} \cup \underline{A}_{s+1} \cup \ldots \cup \underline{A}_{k}\right)$ since $q_{i} \neq 0$ for all $i \leq i_{k-1}$. Hence the above relation is the unique relation in the given polytope, which must be a basic polytope.

Lemma 4.1.2. $\underline{P}_{s}^{E}=\operatorname{conv}\left(\left\{\underline{A}_{0} \cup \ldots \cup \underline{A}_{s} \cup \underline{P} ; \underline{A}_{s+2} \cup \ldots \cup \underline{A}_{k} \cup \underline{Q}\right\}:\left\langle\underline{A}_{s+1}\right\rangle\right)$ for $s=0, \ldots, k-2$ is a set of maximal elementary polytopes such that:

(i) All of the elementary polytopes have the same basic polytope $\mathbf{P}^{\mathbf{B}_{s}}$. 
(ii) For every polytope $\mathbf{P}^{\prime}=\operatorname{conv}\left(\underline{A}_{0} \cup \ldots \cup \underline{A}_{s} \cup \underline{P} ; \underline{A}_{s+2} \cup \ldots \underline{A}_{k} \cup \underline{Q}: *\right)$ in $\underline{P}_{s}^{E}$ (* is an element of $\left.\left\langle A_{s+1}\right\rangle\right)$ the canonical triangulation

$$
\left\langle A_{0} \cup \ldots \cup \underline{A}_{s} \cup \underline{P}\right\rangle\left\{\underline{A}_{s+2} \cup \ldots \cup \underline{A}_{k} \cup \underline{Q}\right\}\{*\}
$$

of $\mathbf{P}^{\prime}$ is a subcomplex of $\mathbf{T}_{s}$.

(iii) For any polytope $\alpha$ belonging to $\operatorname{Star}\left(\left\{\underline{A}_{s+2} \cup \ldots \cup \underline{A}_{k} \cup \underline{Q}\right\}: \mathbf{T}_{s}\right\}$ there exists a polytope $\mathbf{P}^{\prime} \in \underline{P}_{s}^{E}$ such that $\alpha \subset \mathbf{P}^{\prime}$.

Proof. Let $\mathbf{P}^{\prime}=\operatorname{conv}\left(\underline{A}_{0} \cup \ldots \cup \underline{A}_{s} \cup \underline{P} ; \underline{A}_{s+2} \cup \ldots \cup \underline{A}_{k} \cup \underline{Q}: *\right)$ be a polytope in $\underline{P}_{s}^{E}$. Note that $Q$ is not in the face $\operatorname{conv}\left(\underline{A}_{0} \cup \ldots \cup \underline{A}_{s} \cup \underline{A}_{s+2} \cup \ldots \cup \underline{A}_{k} \cup *\right)$ of $\Delta$, since $q_{i} \neq 0$ for all $i_{s-1}+1 \leq i \leq i_{s}$. Therefore $Q$ is independent of the set of vertices of $\operatorname{conv}\left(\underline{A}_{0} \cup \ldots \cup \underline{A}_{s} \cup \underline{A}_{s+2} \cup \ldots \cup \underline{A}_{k} \cup *\right)$. Hence there is the only one relation on the set of vertices of $\mathbf{P}^{\prime}$. By the previous lemma $\mathbf{P}^{\prime}$ is an elementary polytope with the basic polytope $\mathbf{P}_{s}^{\mathrm{B}}$.

(ii) follows from the fact that

$$
\begin{aligned}
\left\langle\underline{A}_{0} \cup \ldots \cup \underline{A}_{s} \cup \underline{P}\right\rangle\left\{\underline{A}_{s+2} \cup \ldots \underline{A}_{k} \cup \underline{Q}\right\}\left\langle\underline{A}_{s+1}\right\rangle \\
=\left\{\underline{A}_{0} \cup \ldots \cup \underline{A}_{s}\right\}\left\langle\underline{A}_{s+1}\right\rangle\left\{\underline{A}_{s+2} \cup \ldots \cup \underline{A}_{k}\right\}\{\underline{Q}\} \\
\quad \cup\left\langle\underline{A}_{0} \cup \ldots \cup \underline{A}_{s}\right\rangle\left\langle\underline{A}_{s+1}\right\rangle\left\{\underline{A}_{s+2} \cup \ldots \cup \underline{A}_{k}\right\}\{\underline{P}\}\{\underline{Q}\}
\end{aligned}
$$

is a subset of $\mathbf{T}_{s}$.

(iii) can be deduced from the fact that

$$
\begin{aligned}
\operatorname{Star} & \left(\left\{\underline{A}_{s+2} \cup \ldots \cup \underline{A}_{k} \cup \underline{Q}\right\} ; \mathbf{T}_{s}\right) \\
& =\left\langle\underline{A}_{0} \cup \ldots \cup \underline{A}_{s} \cup \underline{P}\right\rangle\left\langle\underline{A}_{s+1}\right\rangle\left\{\underline{A}_{s+2} \cup \ldots \cup \underline{A}_{k}\right\}\{\underline{Q}\} \\
& \subset \operatorname{conv}\left(\underline{A}_{0} \cup \ldots \cup \underline{A}_{s} \cup \underline{A}_{s+2} \cup \ldots \cup \underline{A}_{k} \cup \underline{P} \cup \underline{Q}\right)\left\langle\underline{A}_{s+1}\right\rangle .
\end{aligned}
$$

By Lemmas 4.0 and 4.1.2 and since $\mathbf{T}_{s}$ is a triangulation, we get that the set $\underline{P}_{s}^{E}$ determines an elementary transformation. It changes the set

$$
\left\langle\underline{A}_{0} \cup \ldots \cup \underline{A}_{s} \cup \underline{P}\right\rangle\left\{\underline{A}_{s+2} \cup \ldots \cup \underline{A}_{k} \cup \underline{Q}\right\}\left\langle\underline{A}_{s+1}\right\rangle \subset \mathbf{T}_{s}
$$

into a set $\left\{\underline{A}_{0} \cup \ldots \cup \underline{A}_{s} \cup \underline{P}\right\}\left\langle\underline{A}_{s+2} \cup \ldots \cup \underline{A}_{k} \cup Q\right\rangle\left\langle\underline{A}_{s+1}\right\rangle \subset \mathbf{T}_{s+1}$, and transforms the triangulation $\mathbf{T}_{s}$ into $\mathbf{T}_{s+1}$. In particular the set $\mathbf{T}_{s+1}$ is a triangulation.

Lemma 4.1 is proved.

To complete the proof of Proposition 4A, note that all the considered triangulations restricted to $\partial \Delta$ are equal to $\partial \Delta_{(P, Q)}$.

Corollary 4.1.3. Each basic polytope in the above elementary equivalence is of the form

$$
\left(\mathbf{P}^{\mathbf{B}}\right)^{\prime}=\operatorname{conv}\left(A_{i_{1}}, \ldots, A_{i_{h}}, P ; A_{i_{h+1}}, \ldots, A_{i_{g}}, Q\right)
$$

for some subset $\left\{i_{1}, \ldots, i_{g}\right\} \subset\{1, \ldots, n\}$ where $0 \leq h<g$.

Proof of Proposition $4 B$. Pair $\left(\mathbf{P}^{\mathbf{B}} ;\{Q\}\right)$, where $\mathbf{P}^{\mathbf{B}}$ is a basic polytope and $Q \in$ $\mathbf{P}^{\mathrm{B}}$.

Consider two projections resp. on the affine space aff $\left(A_{0}, \ldots, A_{n}\right)$ in direction $\operatorname{aff}\left(B_{0}, \ldots, B_{m}\right)$ and vice versa. Let $r_{0}, r_{1}$ be their restriction to $\mathbf{P}^{\mathbf{B}}$. We have

$$
\begin{gathered}
r_{0}: \mathbf{P}^{\mathbf{B}} \longrightarrow \Delta\left(A_{0}, \ldots, A_{n}\right), \\
r_{1}: \mathbf{P}^{\mathbf{B}} \longrightarrow \Delta\left(B_{0}, \ldots, B_{m}\right), \\
r_{0}\left(\sum a_{i} A_{i}+\sum b_{j} B_{j}\right)=\sum a_{i} A_{i}+\left(\sum b_{i}\right) P, \\
r_{i}\left(\sum a_{i} A_{i}+\sum b_{j} B_{j}\right)=\left(\sum a_{i}\right) P+\sum b_{i} B_{i},
\end{gathered}
$$


where $P:=\Delta\left(A_{0}, \ldots, A_{n}\right) \cap \Delta\left(B_{0}, \ldots, B_{m}\right)$.

Denote $r_{0}(Q)$ by $Q_{0}$, and $r_{1}(Q)$ by $Q_{1}$. For the pairs $\left(\Delta\left(A_{0}, \ldots, A_{n}\right):\left\{Q_{0}, P\right\}\right)$; $\left(\Delta\left(B_{0}, \ldots, B_{m}\right):\left\{Q_{i}, P\right\}\right)$ choose notations as in the previous case: $\underline{A}:=$ $\left\{A_{0}, \ldots, A_{n}\right\}=\underline{A}_{0} \cup \ldots \cup \underline{A}_{l}$ and $\underline{B}:=\left\{B_{0}, \ldots, B_{m}\right\}=\underline{B}_{0} \cup \ldots \cup \underline{B}_{d}$. Set $\underline{Q}:=$ $\{Q\} ;$ and $\underline{P}:=\{P\}$

We shall represent triangulations of the pair $\left(\mathbf{P}^{\mathbf{B}} ; P\right)$ as elements of $S E T^{2}(V)$, where $V=\left\{A_{0}, \ldots, A_{n}, B_{0}, \ldots, B_{m}, Q\right\}$. For instance $\mathbf{B}=\langle\underline{A}\rangle\{\underline{B}\}$.

If $P=Q$ then $\mathbf{A}_{(Q)}=\mathbf{B}_{(Q)}=\langle\underline{A}\rangle\langle\underline{B}\rangle\{\underline{Q}\}$, and we are done. Assume $P \neq Q$. Consider the following cases.

$$
\begin{aligned}
& \text { 1. } Q \notin \partial \mathbf{P}^{\mathbf{B}}: \quad \text { (a) } Q \notin \Delta\left(B_{0}, \ldots, B_{m}\right), \\
& \text { 2. } Q \in \partial \mathbf{P}^{\mathbf{B}}: \quad \text { (a) } Q \in \partial \Delta\left(B_{0}, \ldots, B_{m}\right), \\
& \\
& \text { (b) } Q \in \partial \Delta\left(A_{0}, \ldots, A_{n}\right), \\
& \text { (c) } Q \in \partial \mathbf{P}^{\mathbf{B}} \backslash \partial \Delta\left(B_{0}, \ldots, B_{m}\right) \backslash \partial \Delta\left(A_{0}, \ldots, A_{n}\right) .
\end{aligned}
$$

Case 1a. We then have $Q \in \operatorname{int} \Delta\left(B_{0}, \ldots, B_{m}, A_{i_{0}}, \ldots, A_{i_{p}}\right)$ for some $i_{0}, \ldots, i_{p}$. Then $Q_{0} \in \operatorname{int} \Delta\left(P, A_{i_{0}}, \ldots, A_{i_{p}}\right)=\operatorname{int} \Delta\left(\underline{P}, \underline{A}_{0} \cup \ldots \cup \underline{A}_{l-1}\right)$ (as in the $\left(0,{ }^{*}\right)$ case). Hence $\left\{A_{i_{0}}, \ldots, A_{i_{p}}\right\}=\underline{A}_{0} \cup \ldots \cup \underline{A}_{l-1}$. Finally, $Q \in \operatorname{int} \Delta\left(\underline{B}, \underline{A}_{0} \cup \ldots \cup \underline{A}_{l-1}\right)$. We obtain $\mathbf{B}_{(Q)}$ from $\mathbf{B}=\langle\underline{A}\rangle\{\underline{B}\}$ by replacing

$$
\left\{\underline{B} \cup \underline{A}_{0} \cup \ldots \cup \underline{A}_{l-1}\right\}\left\langle\underline{A}_{l}\right\rangle
$$

with

$$
\begin{aligned}
\left\langle\underline{A}_{0} \cup \ldots \cup \underline{A}_{l-1} \cup \underline{B}\right\rangle\left\langle\underline{A}_{l}\right\rangle\{Q\} \\
\quad=\left\langle\underline{A}_{0} \cup \ldots \cup \underline{A}_{l-1}\right\rangle\left\langle\underline{A}_{l}\right\rangle\{\underline{B}\}\{\underline{Q}\} \\
\quad \cup\left\{\underline{A}_{0} \cup \ldots \cup \underline{A}_{l-1}\right\}\left\langle\underline{A}_{l}\right\rangle\langle\underline{B}\rangle\{\underline{Q}\} .
\end{aligned}
$$

Hence

$$
\begin{gathered}
\mathbf{B}_{(Q)}=\left\langle\underline{A}_{0} \cup \ldots \cup \underline{A}_{l-1}\right\rangle\left\{\underline{A}_{l}\right\}\{\underline{B}\} \cup\left\langle\underline{A}_{0} \cup \ldots \cup \underline{A}_{l-1}\right\rangle\left\langle\underline{A}_{l}\right\rangle\{\underline{B}\}\{\underline{Q}\} \\
\cup\left\{\underline{A}_{0} \cup \ldots \cup \underline{A}_{l-1}\right\}\left\langle\underline{A}_{l}\right\rangle\langle\underline{B}\rangle\{\underline{Q}\} .
\end{gathered}
$$

Since $Q \in \operatorname{int} \mathbf{P}^{\mathbf{B}}$ we have $\mathbf{P}^{\mathbf{B}}(Q)=\langle\underline{A}\rangle\langle\underline{B}\rangle\{Q\}$.

Case 1b. $\mathbf{B}_{(Q)}$ is obtained from $\mathbf{B}$ by changing all its simplices into the set of simplices $\langle\underline{A}\rangle\langle\underline{B}\rangle\{\underline{Q}\}$. Then $\mathbf{P}^{\mathbf{B}}{ }_{(Q)}=\langle\underline{A}\rangle\langle\underline{B}\rangle\{Q\}$, as in the previous case.

Case 2a. $Q=\bar{Q}_{1} \in$ int $\Delta\left(\underline{B}_{0} \cup \ldots \cup \underline{B}_{d-1}\right)$. We insert the set of all simplices from $\langle\underline{A}\rangle\{\underline{B}\}$ into the set $\mathbf{B}_{(Q)}=\langle\underline{A}\rangle\left\langle\underline{B}_{0} \cup \ldots \cup \underline{B}_{d-1}\right\rangle\left\{\underline{B}_{d}\right\}\{Q\}$.

Note also that $\mathbf{P}^{\mathbf{B}}{ }_{(Q)}=\mathbf{B}_{(Q)}=\langle\underline{A}\rangle\left\langle\underline{B}_{0} \cup \ldots \cup \underline{B}_{d-1}\right\rangle\left\{\underline{B}_{d}\right\}\{Q\}$.

Case 2b. $Q=Q_{0} \in$ int $\Delta\left(\underline{A}_{0} \cup \ldots \cup \underline{A}_{l-1}\right)$. We replace $\left\{\underline{A}_{0} \cup \ldots \cup \underline{A}_{l-1}\right\}\left\langle\underline{A}_{l}\right\rangle\{\underline{B}\}$ by $\left\langle\underline{A}_{0} \cup \ldots \cup \underline{A}_{l-1}\right\rangle\left\langle\underline{A}_{l}\right\rangle\{\underline{B}\}\{Q\}$ and get the triangulation

$\mathbf{B}_{(Q)}=\left\langle\underline{A}_{0} \cup \ldots \cup \underline{A}_{l-1}\right\rangle\left\langle\underline{A}_{l}\right\rangle\{\underline{B}\}\{\underline{Q}\} \cup\left\langle\underline{A}_{0} \cup \ldots \cup \underline{A}_{l-1}\right\rangle\left\{\underline{A}_{l}\right\}\{\underline{B}\}$,

$$
\mathbf{P}^{\mathbf{B}}{ }_{(Q)}=\left\langle\underline{A}_{0} \cup \ldots \cup \underline{A}_{l-1}\right\rangle\left\{\underline{A}_{l}\right\}\langle\underline{B}\rangle\{Q\} .
$$

Case 2c. By taking projections $r_{0}, r_{1}$ we get that

$$
Q \in \operatorname{int} \Delta\left(\underline{A}_{0} \cup \ldots \cup \underline{A}_{l-1} \cup \underline{B}_{0} \cup \ldots \cup \underline{B}_{d-1}\right) .
$$


We replace the subsets $\left\{\underline{A}_{0} \cup \ldots \cup \underline{A}_{l-1} \cup \underline{B}\right\}\left\langle\underline{A}_{l}\right\rangle$ of $\langle\underline{A}\rangle\{B\}$ with the set

$$
\begin{aligned}
&\left\langle\underline{A}_{0} \cup \ldots \cup \underline{A}_{l-1} \cup \underline{B}_{0} \cup \ldots \cup \underline{B}_{d-1}\right\rangle\left\langle\underline{A}_{l}\right\rangle\left\{\underline{B}_{d}\right\}\{\underline{Q}\} \\
& \quad=\left\langle\underline{A}_{0} \cup \ldots \cup \underline{A}_{l-1}\right\rangle\left\langle\underline{A}_{l}\right\rangle\{\underline{B}\}\{\underline{Q}\} \\
& \cup\left\{\underline{A}_{0} \cup \ldots \cup \underline{A}_{l-1}\right\}\left\langle\underline{A}_{l}\right\rangle\left\langle\underline{B}_{0} \cup \ldots \cup \underline{B}_{d-1}\right\rangle\left\{\underline{B}_{d}\right\}\{\underline{Q}\}
\end{aligned}
$$

and get the triangulation

$$
\begin{aligned}
& \mathbf{B}_{(Q)}=\left\langle\underline{A}_{0} \cup \ldots \cup \underline{A}_{l-1}\right\rangle\left\{\underline{A}_{l}\right\}\{\underline{B}\} \\
& \cup\left\langle\underline{A}_{0} \cup \ldots \cup \underline{A}_{l-1}\right\rangle\left\langle\underline{A}_{l}\right\rangle\{\underline{B}\}\{\underline{Q}\} \\
& \cup\left\{\underline{A}_{0} \cup \ldots \cup \underline{A}_{l-1}\right\}\left\langle\underline{A}_{l}\right\rangle\left\langle\underline{B}_{0} \cup \ldots \cup \underline{B}_{d-1}\right\rangle\left\{\underline{B}_{d}\right\}\{\underline{Q}\}, \\
& \mathbf{P}_{(Q)}^{\mathbf{B}}=\langle\underline{A}\rangle\langle\underline{B}\rangle\{\underline{Q}\} \backslash\left\{\underline{A}_{0} \cup \ldots \cup \underline{A}_{l-1}\right\}\left\{\underline{B}_{0} \cup \ldots \cup \underline{B}_{d-1}\right\}\left\langle\underline{A}_{l}\right\rangle\left\langle\underline{B}_{d}\right\rangle\{Q\}
\end{aligned}
$$

Note that in cases $1 \mathrm{~b}$ and $2 \mathrm{a} \mathbf{P}_{(Q)}^{\mathbf{B}}=\mathbf{B}_{(Q)}$. Consider the other cases.

Let $\mathbf{T}_{s}$ denotes the following triangulations:

$$
\begin{aligned}
\mathbf{T}_{s}:= & \left\langle\underline{A}_{0} \cup \ldots \cup \underline{A}_{l-s}\right\rangle\left\{\underline{A}_{l-s+1} \cup \ldots \cup \underline{A}_{s}\right\}\{\underline{B}\} \\
& \cup\left\langle\underline{A}_{0} \cup \ldots \cup \underline{A}_{l-s}\right\rangle\left\langle\underline{A}_{l-s+1} \cup \ldots \cup \underline{A}_{s}\right\rangle\{\underline{B}\}\{\underline{Q}\} \\
& \cup\left\{\underline{A}_{0} \cup \ldots \cup \underline{A}_{l-s}\right\}\left\langle\underline{A}_{l-s+1} \cup \ldots \cup \underline{A}_{s}\right\rangle\langle\underline{B}\rangle\{\underline{Q}\} \backslash S,
\end{aligned}
$$

where

$$
S=\left\{\begin{array}{l}
\emptyset \text { in case 1a, } \\
\left\{\underline{A}_{0} \cup \ldots \cup \underline{A}_{l-1}\right\}\left\langle\underline{A}_{l}\right\rangle\langle\underline{B}\rangle\{\underline{Q}\} \text { in case } 2 \mathrm{~b}, \\
\left\{\underline{A}_{0} \cup \ldots \cup \underline{A}_{l-1}\right\}\left\langle\underline{A}_{l}\right\rangle\left\{\underline{B}_{0} \cup \ldots \cup \underline{B}_{d-1}\right\}\left\langle\underline{B}_{d}\right\rangle\{\underline{Q}\} \text { in case 2c. }
\end{array}\right.
$$

Lemma 4.2. (a) $\mathbf{T}_{1}=B_{(Q)}$.

(b) $\mathbf{T}_{s}$ is a triangulation for $s=1, \ldots, l$.

(c) $\mathbf{T}_{s}$ is el-equivalent to $\mathbf{T}_{s-1}$ for $s=2, \ldots, l$.

(d) $\mathbf{T}_{l}$ is el-equivalent to $\mathbf{P}^{\mathbf{B}}{ }_{(Q)}$.

It follows from the lemma that $\mathbf{B}_{(Q)}$ is el-equivalent to $\mathbf{P}^{\mathbf{B}}{ }_{(Q)}$. By symmetry we have that $\mathbf{A}_{(Q)}$ is el-equivalent to $\mathbf{P}_{(Q)}^{\mathbf{B}}$. Finally the lemma yields the elequivalence of $\mathbf{A}_{(Q)}$ and $\mathbf{B}_{(Q)}$.

Proof. (a) follows just from the definition of $\mathbf{T}_{s}$. In case $2 \mathrm{c}$ by properties $1,2,3$ of our operations we have

$$
\begin{aligned}
\left\{\underline{A}_{0} \cup \ldots \cup \underline{A}_{l-1}\right\}\left\langle\underline{A}_{l}\right\rangle\left\langle\underline{B}_{0} \cup \ldots \cup \underline{B}_{d-1}\right\rangle\left\{\underline{B}_{d}\right\}\{\underline{Q}\} \\
\quad=\left\{\underline{A}_{0} \cup \ldots \cup \underline{A}_{l-1}\right\}\left\langle\underline{A}_{l}\right\rangle\langle\underline{B}\rangle\{\underline{Q}\} \\
\quad \backslash\left\{A_{0} \cup \ldots \cup \underline{A}_{l-1}\right\}\left\langle\underline{A}_{l}\right\rangle\left\{\underline{B}_{0} \cup \ldots \cup \underline{B}_{d-1}\right\}\left\langle\underline{B}_{d}\right\rangle\{\underline{Q}\} .
\end{aligned}
$$

Other cases are tautological. We prove (b) and (c) by induction on $s$.

Lemma 4.2.1. For $s=1, \ldots, l-1$

$$
\mathbf{P}^{\mathbf{B}}:=\operatorname{conv}\left(\underline{A}_{0} \cup \ldots \cup \underline{A}_{l-s-1} \cup \underline{B} ; \underline{A}_{l-s+1} \cup \ldots \cup \underline{A}_{l} \cup \underline{Q}\right)
$$

is a basic polytope.

Proof. By Lemma 4.1.1 $\operatorname{conv}\left(\underline{A}_{0} \cup \ldots \cup \underline{A}_{l-s-1} \cup \underline{P} ; \underline{A}_{l-s+1} \cup \ldots \cup \underline{A}_{l} \cup \underline{Q}_{0}\right)$ is a basic polytope. There exist $a_{j}, p, q>0$ such that $\sum_{j \leq i_{l-s-1}} a_{j}+p=\sum_{j>i_{l-s}} a_{j}+q=1$ and

$$
\sum_{j \leq i_{l-s-1}} a_{j} A_{j}+p P=\sum_{j>i_{l-s}} a_{j} A_{j}+q Q_{0}
$$


By the definition of $P$

$$
P=\sum_{i=0}^{n} p_{i} B_{i}, \quad \text { where } p_{i}>0 \text { and } \sum_{i=0}^{n} p_{i}=1 .
$$

There exist $a_{j}^{\prime}$ and $b_{i}^{\prime}$ such that $Q=\sum a_{i}^{\prime} A_{i}+\sum b_{j}^{\prime} B_{j}$. Then by definition of $Q_{0}$ and $r_{0}$

$Q_{0}=\sum a_{i}^{\prime} A_{i}+\left(\sum b_{j}^{\prime}\right) P=Q-\sum b_{j}^{\prime} B_{j}+\left(\sum b_{j}^{\prime}\right) P=Q-\left(\sum\left(b_{j}^{\prime}-\left(\sum b_{j}^{\prime}\right) p_{j}\right) B_{j}\right)$,

$$
Q_{0}=Q+\sum k_{i} B_{i}, \text { where } \sum k_{i}=0 .
$$

Putting (1) and (2) into (0) we get :

$$
\sum_{j \leq i_{l-s-1}} a_{j} A_{j}+\sum_{i \in I} b_{i} B_{i}=\sum_{j>i_{l-s}} a_{j} A_{j}+q Q+\sum_{i \in I^{\prime}} b_{i} B_{i},
$$

where $b_{i}=\left|p p_{i}-q k_{i}\right|$ and $p p_{i}-q k_{i} \geq 0$ iff $i \in I$. In particular all the coefficients are non-negative, and $I$ and $I^{\prime}$ denote two disjoint sets of indices such that $I \cup I^{\prime} \subseteq$ $\{0, \ldots, m\}$.

The set $I$ is non-empty since some $k_{i}$ in (2) are non-positive. Assume that $I$ is not a proper subset of $\{0, \ldots, m\}$, i.e. $I^{\prime}$ is nonempty. Then the right side of (3) would be a point of the face $\mathbf{P}^{\prime}=\operatorname{conv}\left(A_{j}, B_{i}: j \leq i_{l-s-1}, i \in I\right)$ of $\mathbf{P}^{B}$. If $Q \notin \mathbf{P}^{\prime}$ then by (3) we get that the all points on $\operatorname{RHS} A_{j}, j \geq i_{l-s}, B_{i}, i \in$ $I^{\prime}$, do not belong to the face $\mathbf{P}^{\prime}$ of $\mathbf{P}^{B}$. Since these points belong to $\mathbf{P}^{B}$ and do not belong to $\mathbf{P}^{\prime}$, their convex hull does not intersect $\mathbf{P}^{\prime}$, which contradicts (3). If $Q \in \mathbf{P}^{\prime}$ then $Q_{0} \in \operatorname{conv}\left(A_{j}, P: j \leq i_{l-s-1}\right)$, which is not possible since $\operatorname{conv}\left(\underline{A}_{0} \cup \ldots \cup \underline{A}_{l-s-1} \cup \underline{P}: \underline{A}_{l-s+1} \cup \ldots \cup \underline{A}_{l} \cup \underline{Q}_{0}\right)$ is a basic polytope. Finally $I=\{0, \ldots, m\}$ and $I^{\prime}$ is empty. We can write $(3)$ as

$$
\sum_{j \leq i_{l-s-1}} a_{j} A_{j}+\sum b_{i} B_{i}=\sum_{j>i_{l-s}} a_{j} A_{j}+q Q .
$$

Note also that (4) is the only relation on the set of vertices.

Lemma 4.2.2. $\underline{P}_{s}^{E}=\operatorname{conv}\left(\left\{\underline{A}_{0} \cup \ldots \cup \underline{A}_{l-s-1} \cup \underline{B} ; A_{l-s+1} \cup \ldots \cup \underline{A}_{l} \cup \underline{Q}\right\}\left\langle\underline{A}_{l-1}\right\rangle\right)$ is a set of maximal elementary polytopes such that:

1. All of the elementary polytopes have the same basic polytope $\mathbf{P}^{\mathbf{B}}$.

2. For every polytope $\mathbf{P}^{\prime}=\operatorname{conv}\left(\underline{A}_{0} \cup \ldots \cup \underline{A}_{l-s-1} \cup \underline{B} ; \underline{A}_{l-s-1} \cup \ldots \cup \underline{A}_{l} \cup \underline{Q} ; *\right) \in$ $\underline{P}_{s}^{E}$ the canonical triangulation

$$
\left\{\underline{A}_{0} \cup \ldots \cup \underline{A}_{l-s-1} \cup \underline{B}\right\}\left\langle\underline{A}_{l-s+1} \cup \ldots \cup \underline{A}_{l} \cup \underline{Q}\right\rangle\{*\}
$$

of $\mathbf{P}^{\prime}$ is a subcomplex of $\mathbf{T}_{s}$.

3. For every polytope $\alpha$ belonging to $\operatorname{Star}\left(\left\{\underline{A}_{0} \cup \ldots \cup \underline{A}_{l-s-1} \cup \underline{B}\right\}: \mathbf{T}_{s}\right)$ there exists a polytope $\mathbf{P}^{\prime} \in \underline{P}_{s}^{E}$ such that $\alpha \subset \mathbf{P}^{\prime}$.

Proof. As for Lemma 4.1.2.

By Lemmas 4.0 and 4.2 .2 , and since $\mathbf{T}_{s}$ is a triangulation, the set $\underline{P}_{s}^{E}$ determines an elementary transformation. It changes the set

$$
\left\{\underline{A}_{0} \cup \ldots \cup \underline{A}_{l-s-1} \cup \underline{B}\right\}\left\langle\underline{A}_{l-s+1} \cup \ldots \cup \underline{A}_{l} \cup \underline{Q}\right\rangle\left\langle\underline{A}_{l-s}\right\rangle \subseteq \mathbf{T}_{s}
$$

into

$$
\left\langle\underline{A}_{0} \cup \ldots \cup \underline{A}_{l-s-1} \cup \underline{B}\right\rangle\left\{\underline{A}_{l-s+1} \cup \ldots \cup \underline{A}_{l} \cup \underline{Q}\right\}\left\langle\underline{A}_{l-s}\right\rangle \subseteq \mathbf{T}_{s+1}
$$


transforming $\mathbf{T}_{s}$ to the triangulation $\mathbf{T}_{s+1}$.

The elementary transformation with the basic complex

$$
\operatorname{conv}\left(\underline{B}: \underline{Q} \cup \underline{A}_{1} \cup \ldots \cup \underline{A}_{l}\right)\left\langle\underline{A}_{0}\right\rangle
$$

changes the set

$$
\left\langle\underline{A}_{0}\right\rangle\left\langle A_{1} \cup \ldots \cup \underline{A}_{l}\right\rangle\{\underline{B}\}\{\underline{Q}\} \cup\left\{\underline{A}_{1} \cup \ldots \cup \underline{A}_{l}\right\}\{\underline{B}\} \subseteq \mathbf{T}_{l}
$$

into

$$
\left\langle\underline{A}_{0}\right\rangle\left\{\underline{A}_{1} \cup \ldots \cup \underline{A}_{l}\right\}\langle\underline{B}\rangle\{\underline{Q}\} \subseteq \mathbf{P}^{\mathbf{B}}(Q)
$$

and transforms $\mathbf{T}_{l}$ to $\mathbf{P}_{(Q)}^{B}$. Lemma 4.2 is proved.

To complete the proof of Proposition 4B, note that all the considered triangulations restricted to the boundary $\partial \mathbf{P}^{B}$ of $\mathbf{P}^{B}$ are equal to $\left(\partial \mathbf{P}^{B}\right)_{(Q)}$.

Corollary 4.2.3. Let $Q \in \operatorname{int} \Delta\left(A_{0}, \ldots, A_{n}\right)$. Then $\mathbf{B}_{(Q)}$ and $\mathbf{A}_{(Q)}$ differ by a succesion of elementary transformations with basic polytopes of the form

$$
\operatorname{conv}\left(A_{i_{1}}, \ldots A_{i_{h}}, Q ; B_{0}, \ldots B_{m}, A_{i_{h+1}}, \ldots, A_{i_{g}}\right)
$$

for some subset $\left\{i_{1}, \ldots, i_{g}\right\} \subset\{0, \ldots, n\}$ where $h \leq g$

Proof. It follows from the previous proof that $\mathbf{B}_{(Q)}$ and $\mathbf{P}_{(Q)}^{\mathbf{B}}$ differ by elementary transformations with basic polytopes of the form

$$
\operatorname{conv}\left(A_{i_{1}}, \ldots, A_{i_{h}}, Q ; B_{0}, \ldots, B_{m}, A_{i_{h+1}}, \ldots, A_{i_{g}}\right)
$$

where $h \leq g$.

In case la we have that $\mathbf{P}_{(Q)}^{\mathbf{B}}=\mathbf{B}_{(Q)}$. The case $Q \in \operatorname{int} \Delta\left(A_{0}, \ldots, A_{n}\right)$ is symmetric to case 1a. Thus we have $\mathbf{P}_{(Q)}^{\mathbf{B}}=\mathbf{A}_{(Q)}$.

Finally, $\mathbf{B}_{(Q)}$ and $\mathbf{A}_{(Q)}$ differ by elementary transformations with basic polytopes of the given form.

Proof of Proposition $4 C$. Consider a pair $\left(\mathbf{P}^{\mathbf{E}} ; Q\right)$, where $\mathbf{P}^{\mathbf{E}}, Q$ are as in part $\mathbf{C}$ of Proposition 4.

Let $\underline{C}=\left\{C_{1}, \ldots, C_{r}\right\}, \underline{A}=\left\{A_{0}, \ldots, A_{n}\right\}, \underline{B}=\left\{B_{0}, \ldots, B_{m}\right\}, Q=\{Q\}$.

Assume that $Q \in \Delta\left(C_{1}, \ldots, C_{r}\right)$. Thus $\mathbf{B}_{(Q)}=\langle\underline{A}\rangle\{\underline{B}\}\langle\underline{C}\rangle\{\underline{Q}\}, \mathbf{A}_{(Q)}=$ $\{\underline{A}\}\langle\underline{B}\rangle\langle\underline{C}\rangle\{\underline{Q}\}$, and $\mathbf{A}_{(Q)}$ differs from $\mathbf{B}_{(Q)}$ by an elementary transformation on the basic complex $\{\underline{A} \cup \underline{B}\}\langle\underline{C}\rangle\{Q\}$; and we are done.

Thus now we assume that $Q \notin \Delta\left(C_{1}, \ldots, C_{r}\right)$. Let $p$ be the projection from the face $\Delta\left(C_{1}, \ldots, C_{r}\right)$ of $\mathbf{P}^{\mathbf{E}}$ onto the basic polytope $\mathbf{P}^{\mathbf{B}}=\operatorname{conv}\left(A_{0}, \ldots, A_{n}\right.$ : $\left.B_{0}, \ldots, B_{m}\right)$. Write in affine coordinates

$$
\begin{gathered}
p: \mathbf{P}^{\mathbf{E}} \backslash \Delta\left(C_{1}, \ldots, C_{P}\right) \longrightarrow \mathbf{P}^{\mathbf{B}}, \\
p\left(\sum a_{i} A_{i}+\sum b_{j} B_{j}+\sum c_{k} C_{k}\right)=\sum\left(a_{i} / c\right) A_{i}+\sum\left(b_{j} / c\right) B_{j},
\end{gathered}
$$

where $c=1-\sum c_{k}>0$.

Let $Q^{\prime}=p(Q)$. For $Q^{\prime} \in \mathbf{P}^{B}$ find the sets $\underline{A}_{i}$ and $\underline{B}_{j}$ as in the case $4 \mathrm{~B}$. This allows us to represent triangulations of the considered pair as elements of $\operatorname{SET}^{2}(V)$ where $V=\left\{A_{0}, \ldots, A_{n}, B_{0}, \ldots, B_{m}, C_{1}, \ldots, C_{r}, Q\right\}$. (See $4 \mathrm{~A}$ and $4 \mathrm{~B}$.) 
We represent the triangulation $\mathbf{B}_{(Q)}$. We have $\mathbf{B}=\langle\underline{A}\rangle \cdot\{\underline{B}\} \cdot\{\underline{C}\}$. Consider several cases.

1. $Q^{\prime} \notin \partial \mathbf{P}^{\mathrm{B}}: \quad$ (a) $Q^{\prime} \notin \Delta\left(B_{0}, \ldots, B_{m}\right)$,

(b) $Q^{\prime} \in \operatorname{int} \Delta\left(B_{0}, \ldots, B_{m}\right)$;

2. $Q^{\prime} \in \partial \mathbf{P}^{\mathbf{B}}:(a) \quad Q^{\prime} \in \partial \Delta\left(B_{0}, \ldots, B_{m}\right)$,

(b) $Q^{\prime} \in \partial \Delta\left(A_{0}, \ldots, A_{n}\right)$,

(c) $Q^{\prime} \in \partial \mathbf{P}^{\mathbf{B}} \backslash \partial \Delta\left(B_{0}, \ldots, B_{m}\right) \backslash \partial \Delta\left(A_{0}, \ldots, A_{n}\right)$.

Case 1a. As before, $Q^{\prime} \in \operatorname{int} \Delta\left(\underline{B}, \underline{A}_{0}, \cup \ldots \cup \underline{A}_{l-1}\right)$; hence

$$
Q \in \operatorname{int} \Delta\left(\underline{B}, \underline{A} \cup \ldots \cup \underline{A}_{l-1}, \underline{C}\right) .
$$

$\mathbf{B}_{(Q)}$ is obtained from $\mathbf{B}=\langle\underline{A}\rangle\{\underline{B}\}\{\underline{C}\}$ by changing $\left\{\underline{B} \cup \underline{A}_{0} \cup \ldots \cup \underline{A}_{l-1} \cup \underline{C}\right\}\left\langle\underline{A}_{l}\right\rangle$ into

$$
\begin{aligned}
&\left\langle\underline{A}_{0} \cup \ldots \cup \underline{A}_{l-1} \cup \underline{B} \cup \underline{C}\right\rangle\left\langle\underline{A}_{l}\right\rangle\{Q\} \\
&=\left\langle\underline{A}_{0} \cup \ldots \cup \underline{A}_{l-1}\right\rangle\left\langle A_{l}\right\rangle\{\underline{B}\}\{\underline{C}\}\{\underline{Q}\} \\
& \cup\left\{\underline{A}_{0} \cup \ldots \cup \underline{A}_{l-1}\right\}\left\langle A_{l}\right\rangle\langle\underline{B}\rangle\{\underline{C}\}\{\underline{Q}\} \\
& \cup\left\{\underline{A}_{0} \cup \ldots \cup \underline{A}_{l-1}\right\}\left\langle A_{l}\right\rangle\{\underline{B}\}\langle\underline{C}\rangle\{\underline{Q}\} .
\end{aligned}
$$

Hence:

$$
\begin{aligned}
\mathbf{B}_{(Q)}= & \left\langle\underline{A}_{0} \cup \ldots \cup \underline{A}_{l-1}\right\rangle\left\{\underline{A}_{l}\right\}\{\underline{B}\}\{\underline{C}\} \\
& \cup\left\langle\underline{A}_{0} \cup \ldots \cup \underline{A}_{l-1}\right\rangle\left\langle A_{l}\right\rangle\{\underline{B}\}\{\underline{C}\}\{\underline{Q}\} \\
& \cup\left\{\underline{A}_{0} \cup \ldots \cup \underline{A}_{l-1}\right\}\left\langle A_{l}\right\rangle\langle\underline{B}\rangle\{\underline{C}\}\{\underline{Q}\} \\
& \cup\left\{\underline{A}_{0} \cup \ldots \cup \underline{A}_{l-1}\right\}\left\langle A_{l}\right\rangle\{\underline{B}\}\langle\underline{C}\rangle\{\underline{Q}\} .
\end{aligned}
$$

Case 1b. $\left.Q^{\prime} \in \operatorname{int} \Delta(\underline{B}) \Longrightarrow Q \in \operatorname{int} \Delta(\underline{B} \cup \underline{C})\right)$. $\mathbf{B}_{(Q)}$ is obtained from $\mathbf{B}$ by changing the set of all its simplices into the set

$$
\mathbf{B}_{(Q)}=\langle\underline{A}\rangle\langle\underline{B}\rangle\{\underline{C}\}\{\underline{Q}\} \cup\langle\underline{A}\rangle\{\underline{B}\}\langle\underline{C}\rangle\{\underline{Q}\} .
$$

Case 2a. $Q^{\prime} \in \operatorname{int} \Delta\left(\underline{B}_{0} \cup \ldots \cup \underline{B}_{d-1}\right)$. Hence $Q \in \operatorname{int} \Delta\left(\underline{B}_{0} \cup \ldots \cup \underline{B}_{d-1} \cup \underline{C}\right)$. We replace the set of all simplices $\langle\underline{A}\rangle\{\underline{B}\}\{\underline{C}\}$ by the set

$$
\mathbf{B}_{(Q)}=\langle\underline{A}\rangle\left\langle\underline{B}_{0} \cup \ldots \cup \underline{B}_{d-1}\right\rangle\left\{\underline{B}_{d}\right\}\{\underline{C}\}\{Q\} \cup\langle\underline{A}\rangle\{\underline{B}\}\langle\underline{C}\rangle\{\underline{Q}\} .
$$

Case 2b. Then $Q \in \operatorname{int} \Delta\left(\underline{A}_{0} \cup \ldots \cup \underline{A}_{l-1} \cup \underline{C}\right\}$. We replace

$$
\left\{A_{0} \cup \ldots \cup \underline{A}_{l-1}\right\}\left\langle\underline{A}_{l}\right\rangle\{\underline{B}\}\{\underline{C}\} \subset\langle\underline{A}\rangle\{\underline{B}\}\{\underline{C}\}
$$

by

$$
\begin{aligned}
\left\langle\underline{A}_{0} \cup \ldots \cup \underline{A}_{l-1}\right\rangle\left\langle\underline{A}_{l}\right\rangle\{\underline{B}\}\{\underline{C}\}\{\underline{Q}\} \\
\cup\left\{\underline{A}_{0} \cup \ldots \cup \underline{A}_{l-1}\right\}\left\langle\underline{A}_{l}\right\rangle\{\underline{B}\}\langle\underline{C}\rangle\{\underline{Q}\}
\end{aligned}
$$

to get

$$
\begin{aligned}
\mathbf{B}_{(Q)}= & \left\langle\underline{A}_{0} \cup \ldots \cup \underline{A}_{l-1}\right\rangle\left\{\underline{A}_{l}\right\}\{\underline{B}\}\{\underline{C}\} \\
& \cup\left\langle\underline{A}_{0} \cup \ldots \cup \underline{A}_{l-1}\right\rangle\left\langle\underline{A}_{l}\right\rangle\{\underline{B}\}\{\underline{C}\}\{\underline{Q}\} \\
& \cup\left\{\underline{A}_{0} \cup \ldots \cup \underline{A}_{l-1}\right\}\left\langle\underline{A}_{l}\right\rangle\{\underline{B}\}\langle\underline{C}\rangle\{\underline{Q}\} .
\end{aligned}
$$

Case 2c. Then $Q^{\prime} \in \operatorname{int} \Delta\left(\underline{A}_{0} \cup \ldots \cup \underline{A}_{l-1} \cup \underline{B}_{0} \cup \ldots \cup \underline{B}_{d-1}\right)$. Hence

$$
Q \in \operatorname{int} \Delta\left(\underline{A}_{0} \cup \ldots \cup \underline{A}_{l-1} \cup \underline{B}_{0} \cup \ldots \cup \underline{B}_{d-1} \cup \underline{C}\right) .
$$


We replace the set $\left\{\underline{A}_{0} \cup \ldots \cup \underline{A}_{l-1} \cup \underline{B} \cup \underline{C}\right\}\left\langle\underline{A}_{l}\right\rangle$ by

$$
\begin{aligned}
&\left\langle\underline{A}_{0} \cup \ldots \cup \underline{A}_{l-1} \cup \underline{B}_{0} \cup \ldots \cup \underline{B}_{d-1} \cup \underline{C}\right\rangle\left\langle\underline{A}_{l}\right\rangle\left\{\underline{B}_{d}\right\}\{\underline{Q}\} \\
&=\left\langle\underline{A}_{0} \cup \ldots \cup \underline{A}_{l-1}\right\rangle\left\langle\underline{A}_{l}\right\rangle\{\underline{B}\}\{\underline{C}\}\{\underline{Q}\} \\
& \cup\left\{\underline{A}_{0} \cup \ldots \cup \underline{A}_{l-1}\right\}\left\langle\underline{A}_{l}\right\rangle\left\{\underline{B}_{\langle}\right\}\langle\underline{C}\rangle\{\underline{Q}\} \\
& \cup\left\{\underline{A}_{0} \cup \ldots \cup \underline{A}_{l-1}\right\}\left\langle\underline{A}_{l}\right\rangle\left\langle\underline{B}_{0} \cup \ldots \cup \underline{B}_{d-1}\right\rangle\left\{\underline{B}_{d}\right\}\{\underline{C}\}\{\underline{Q}\}
\end{aligned}
$$

to get

$$
\begin{aligned}
\mathbf{B}_{(Q)}= & \left\langle\underline{A}_{0} \cup \ldots \cup \underline{A}_{l-1}\right\rangle\left\{\underline{A}_{l}\right\}\{\underline{B}\}\{\underline{C}\} \\
& \cup\left\langle\underline{A}_{0} \cup \ldots \cup \underline{A}_{l-1}\right\rangle\left\langle\underline{A}_{l}\right\rangle\{\underline{B}\}\{\underline{C}\}\{\underline{Q}\} \\
& \cup\left\{\underline{A}_{0} \cup \ldots \cup \underline{A}_{l-1}\right\}\left\langle\underline{A}_{l}\right\rangle\{\underline{B}\}\langle\underline{C}\rangle\{\underline{Q}\} \\
& \cup\left\{\underline{A}_{0} \cup \ldots \cup \underline{A}_{l-1}\right\}\left\langle\underline{A}_{l}\right\rangle\left\langle\underline{B}_{0} \cup \ldots \cup \underline{B}_{d-1}\right\rangle\left\{\underline{B}_{d}\right\}\{\underline{C}\}\{\underline{Q}\} .
\end{aligned}
$$

Let $\mathbf{T}_{B}$ be denote the following triangulation:

$$
\begin{aligned}
\mathbf{T}_{B}:= & \langle\underline{A}\rangle\langle\underline{B}\rangle\{\underline{C}\}\{\underline{Q}\} \cup\langle\underline{A}\rangle\{\underline{B}\}\langle\underline{C}\rangle\{\underline{Q}\} \\
& \text { in cases } 1 \mathrm{a}, 1 \mathrm{~b}, \\
\mathbf{T}_{B}:= & \langle\underline{A}\rangle\left\langle\underline{B}_{0} \cup \ldots \cup \underline{B}_{d-1}\right\rangle\left\{\underline{B}_{d}\right\}\{\underline{C}\}\langle\underline{Q}\rangle \cup\langle\underline{A}\rangle\{\underline{B}\}\langle\underline{C}\rangle\{\underline{Q}\} \\
& \text { in case } 2 \mathrm{a}, \\
\mathbf{T}_{B}:= & \left\langle\underline{A}_{0} \cup \ldots \cup \underline{A}_{l-1}\right\rangle\left\{\underline{A}_{l}\right\}\langle\underline{B}\rangle\{\underline{C}\}\langle\underline{Q}\rangle \cup\langle\underline{A}\rangle\{\underline{B}\}\langle\underline{C}\rangle\{\underline{Q}\} \\
& \text { in case } 2 \mathrm{~b}, \\
\mathbf{T}_{B}:= & \langle\underline{A}\rangle\langle\underline{B}\rangle\{\underline{C}\}\langle\underline{Q}\rangle \cup\langle\underline{A}\rangle\{\underline{B}\}\langle\underline{C}\rangle\{\underline{Q}\} \\
& \backslash\left\{\underline{A}_{0} \cup \ldots \cup \underline{A}_{l-1}\right\}\left\{\underline{B}_{0} \cup \ldots \underline{B}_{d-1}\right\}\left\langle\underline{A}_{l}\right\rangle\left\langle\underline{B}_{d}\right\rangle\{\underline{C}\}\{\underline{Q}\} \\
& \text { in case } 2 \mathrm{c} .
\end{aligned}
$$

We can write it shorter:

$$
\mathbf{T}_{B}=\langle\underline{A}\rangle\langle\underline{B}\rangle\{\underline{C}\}\{\underline{Q}\} \cup\langle\underline{A}\rangle\{\underline{B}\}\langle\underline{C}\rangle\{\underline{Q}\} \backslash S
$$

where

$$
S=\left\{\begin{array}{l}
\emptyset \text { in cases 1a, 1b, i.e. } Q \in \partial \mathbf{P}^{B}, \\
\left\{\underline{A}_{\}}\left\{\underline{B}_{0} \cup \ldots \cup \underline{B}_{d-1}\right\}\left\langle\underline{B}_{d}\right\rangle\{\underline{C}\}\{\underline{Q}\} \text { in case } 2 \mathrm{a},\right. \\
\left\{\underline{A}_{0} \cup \ldots \cup \underline{A}_{l-1}\right\}\left\langle\underline{A}_{l}\right\rangle\langle\underline{B}\rangle\{\underline{C}\}\{\underline{Q}\} \text { in case } 2 \mathrm{~b}, \\
\left\{\underline{A}_{0} \cup \ldots \cup \underline{A}_{l-1}\right\}\left\langle\underline{A}_{l}\right\rangle\left\{\underline{B}_{0} \cup \ldots \cup \underline{B}_{d-1}\right\}\left\langle\underline{B}_{d}\right\rangle\{\underline{C}\}\{\underline{Q}\} \text { in case 2c. }
\end{array}\right.
$$

Note that $\mathbf{T}_{B}=\mathbf{P}^{\mathbf{E}}{ }_{\left(Q, B_{j}\right)}$ for any vertex $B_{j}$ of $\mathbf{P}^{\mathbf{E}}$. By symmetry let $\mathbf{T}_{A}=$ $\mathbf{P}^{\mathbf{E}}{ }_{\left(Q, A_{i}\right)}$. Then $\mathbf{T}_{A}=\langle\underline{A}\rangle\langle\underline{B}\rangle\{\underline{C}\}\{\underline{Q}\} \cup\{\underline{A}\}\langle\underline{B}\rangle\langle\underline{C}\rangle\{\underline{Q}\} \backslash S$

The triangulations $\mathbf{T}_{A}$ and $\mathbf{T}_{B}$ differ by an elementary transformation on the basic complex $\operatorname{conv}(\{\underline{A} ; \underline{B}\}\langle\underline{C}\rangle\{\underline{Q}\})$. Thus it is sufficient to prove the el-equivalence of $\mathbf{T}_{B}$ and $\mathbf{B}_{(Q)}$, and we get the el-equivalence of $\mathbf{A}_{(Q)}$ and $\mathbf{B}_{(Q)}$ by symmetry.

Note that in cases 1.b and 2.a we have that $\mathbf{T}_{B}=\mathbf{B}_{(Q)}$. Consider the other cases. Let $\mathbf{T}_{s}$ denote the following subsets of $\operatorname{SET}^{2}(V)$.

$$
\begin{aligned}
\mathbf{T}_{s}:= & \left\langle\underline{A}_{0} \cup \ldots \cup \underline{A}_{l-s}\right\rangle\left\{\underline{A}_{l-s+1} \cup \ldots \cup \underline{A}_{l}\right\}\{\underline{B}\}\{\underline{C}\} \\
& \cup\left\langle\underline{A}_{0} \cup \ldots \cup \underline{A}_{l-s}\right\rangle\left\langle\underline{A}_{l-s+1} \cup \ldots \cup \underline{A}_{l}\right\rangle\{\underline{B}\}\{\underline{C}\}\{\underline{Q}\} \\
& \cup\left\{\underline{A}_{0} \cup \ldots \cup \underline{A}_{l-s}\right\}\left\langle\underline{A}_{l-s+1} \cup \ldots \cup \underline{A}_{l}\right\rangle\langle\underline{B}\rangle\{\underline{C}\}\{\underline{Q}\} \\
& \cup\left\{\underline{A}_{0} \cup \ldots \cup \underline{A}_{l-s}\right\}\left\langle\underline{A}_{l-s+1} \cup \ldots \cup \underline{A}_{l}\right\rangle\{\underline{B}\}\langle\underline{C}\rangle\{\underline{Q}\} \backslash S .
\end{aligned}
$$

Lemma 4.3. (a) $\mathbf{T}_{1}=\mathbf{B}_{(Q)}$.

(b) $\mathbf{T}_{s}$ is a triangulation for $s=1, \ldots, l$.

(c) $\mathbf{T}_{s}$ is el-equivalent to $\mathbf{T}_{s-1}$ for $s=2, \ldots, l$. 
(d) $\mathbf{T}_{l}$ is el-equivalent to $\mathbf{P}_{(Q)}^{\mathbf{E}}$.

Proof. (a) just follows from the definition.

We prove (b) and (c) by induction on $s$.

Lemma 4.3.1. $\mathbf{P}^{\mathrm{B}}{ }_{s}:=\operatorname{conv}\left(\underline{A}_{0} \cup \ldots \cup \underline{A}_{l-s-1} \cup \underline{B} \cup \underline{C} ; \underline{A}_{l-s+1} \cup \ldots \cup \underline{A_{l}} \cup \underline{Q}\right)$, where $s=1, \ldots, l$, is a basic polytope.

Proof. By Lemma 4.2.1, $\operatorname{conv}\left(\underline{A}_{0} \cup \ldots \cup \underline{A}_{l-s-1} \cup \underline{B} ; \underline{A}_{l-s+1} \cup \ldots \cup \underline{A}_{l} \cup \underline{Q^{\prime}}\right)$ is a basic polytope. Thus

$$
\sum_{j \leq i_{l-s-1}} a_{j} A_{j}+\sum b_{i} B_{i}=\sum_{j>i_{l-s}} a_{j} A_{j}+q Q^{\prime}
$$

where all coefficients are positive. By the definition of $Q^{\prime}$ and of the projection $p$

$$
Q^{\prime}=q^{\prime \prime} Q-\sum k_{i} C_{i}, \quad \text { where } k_{i}>0 \text { and } q^{\prime \prime}>0 .
$$

Putting (1) into (0), we get:

$$
\sum_{j \leq i_{l-s-1}} a_{j} A_{j}+\sum b_{i} B_{i}+\sum q k_{i} C_{i}=\sum_{j>i_{l-s}} a_{j} A_{j}+q q^{\prime \prime} Q .
$$

To complete the proof note that (2) is the only relation on the set of vertices. (If we exclude $Q$ we get an affinely independent set.)

\section{Lemma 4.3.2.}

$$
\underline{P}_{s}^{E}=\operatorname{conv}\left(\left\{\underline{A}_{0} \cup \ldots \cup \underline{A}_{l-s-1} \cup \underline{B} \cup \underline{C} ; \underline{A}_{l-s+1} \cup \ldots \cup \underline{A}_{l} \cup \underline{Q}\right\}\left\langle\underline{A}_{l-s}\right\rangle\{\underline{C}\}\right)
$$

is a set of maximal elementary polytopes such that:

i) All the elementary polytopes have the same basic polytope $\mathbf{P}^{\mathbf{B}}$.

ii) For any polytope

$$
\mathbf{P}^{\prime}=\operatorname{conv}\left(\underline{A}_{0} \cup \ldots \cup \underline{A}_{l-s-1} \cup \underline{B} \cup \underline{C} ; \underline{A}_{l-s+1} \cup \ldots \cup \underline{A}_{l} \cup \underline{Q}: *\right)
$$

in $\underline{P}_{s}^{E}$, where $* \in\left\langle\underline{A}_{l-s}\right\rangle\{\underline{C}\}$, the canonical triangulation of $\mathbf{P}^{\prime}$,

$$
\left\{\underline{A}_{0} \cup \ldots \cup \underline{A}_{l-s-1} \cup \underline{B} \cup \underline{C}\right\} \quad\left\langle\underline{A}_{l-s+1} \cup \ldots \cup \underline{A}_{l} \cup \underline{Q}\right\rangle\{*\},
$$

is a subcomplex of $\mathbf{T}_{s}$.

iii) For every polytope a belonging to $\operatorname{Star}\left(\underline{A}_{0} \cup \ldots \cup \underline{A}_{l-s-1} \cup \underline{B} \cup \underline{C}: \mathbf{T}_{s}\right)$ there exists a polytope $\mathbf{E}^{\prime} \in \underline{P}_{s}^{E}$ such that $\alpha \subset \mathbf{E}^{\prime}$.

Proof. As for Lemma 4.1.2.

By Lemma 4.0 and Lemma 4.3.2 $\underline{P}_{s}^{E}$ determines the elementary transformation which changes

$$
\left\{\underline{A}_{0} \cup \ldots \cup \underline{A}_{l-s-1} \cup \underline{B} \cup \underline{C}\right\}\left\langle\underline{A}_{l-s+1} \cup \ldots \cup \underline{A}_{l} \cup \underline{Q}\right\rangle\left\langle\underline{A}_{l-s}\right\rangle
$$

into

$$
\left\langle\underline{A}_{0} \cup \ldots \cup \underline{A}_{l-s-1} \cup \underline{B} \cup \underline{C}\right\rangle\left\{\underline{A}_{l-s+1} \cup \ldots \cup \underline{A}_{l} \cup \underline{Q}\right\}\left\langle\underline{A}_{l-s}\right\rangle
$$

transforming the triangulation $\mathbf{T}_{s}$ to the triangulation $\mathbf{T}_{s+1}$. This shows points a) b) c) of 4.3 .

The elementary transformation on the basic complex

$$
\operatorname{conv}\left(\underline{B} \cup \underline{C}: \underline{Q} \cup \underline{A}_{1} \cup \ldots \cup \underline{A}_{l}\right)\left\langle\underline{A}_{0}\right\rangle
$$

changes

$$
\left\langle\underline{A}_{0}\right\rangle\left\{\underline{A}_{1} \cup \ldots \cup \underline{A}_{l}\right\}\{\underline{B}\}\{\underline{C}\} \cup\left\langle\underline{A}_{0}\right\rangle\left\langle\underline{A}_{1} \cup \ldots \cup \underline{A}_{l}\right\rangle\{\underline{B}\}\{\underline{C}\} \quad\{\underline{Q}\} \subseteq T_{l}
$$


into:

$$
\begin{aligned}
\left\langle\underline{A}_{0}\right\rangle\left\{\underline{A}_{1} \cup \ldots \cup \underline{A}_{l}\right\}\langle\underline{B}\rangle\{\underline{C}\}\{\underline{Q}\} & \\
& \cup\left\langle\underline{A}_{0}\right\rangle\left\{\underline{A}_{1} \cup \ldots \cup \underline{A}_{l}\right\}\{\underline{B}\}\langle\underline{C}\rangle\{\underline{Q}\} \subseteq \mathbf{P}^{\mathbf{E}}{ }_{(Q)}
\end{aligned}
$$

and transforms $\mathbf{T}_{l}$ to $\mathbf{P}^{\mathbf{E}}(Q)$.

Thus we have proved Lemma 4.3 and, by symmetry, the whole Proposition 4C.

\section{El-Equivalence of Blow-Ups of a Simplicial Complex}

Proposition 5.0. Let $\mathbf{K}$ be simplicial complex, and let $P, Q \in|\mathbf{K}|$. Then:

(a) The triangulations $\mathbf{K}_{(Q, P)}$ and $\mathbf{K}_{(P, Q)}$ are el-equivalent

(b) The elementary transformations realizing the above equivalence do not change the triangulations outside the simplices which contain both $P$ and $Q$.

(c) The elementary transformations are induced (in the sense of section 3) by an elementary transformation of the minimal simplex containing both points $P$, $Q$.

Proof. We consider two cases:

i) $P, Q$ are contained in some simplex $\Delta$ of $\mathbf{K}$.

Assume that $\Delta$ is minimal i.e. $P, Q$ are not contained in any proper face of $\Delta$. By Proposition 4.A $\Delta_{(P, Q)}$ and $\Delta_{(Q, P)}$ are el-equivalent, and any elementary transformation realizing that does not change the triangulations of proper faces of $\Delta$. By Corollary 3.5, Proposition 3.6 and Proposition 3.8 the triangulations $\mathbf{K}\left(\Delta_{(P, Q)}\right)=\mathbf{K}(\Delta)_{(P, Q)}=\mathbf{K}_{(P, Q)}$ and $\mathbf{K}\left(\Delta_{(Q, P)}\right)=\mathbf{K}_{(Q, P)}$ are elequivalent, and the relevant transformations do not change the triangulations outside of $\operatorname{CompStar}(\Delta ; \mathbf{K})$.

ii) $P, Q$ are not contained in any common simplex.

Hence $P \in \operatorname{int} \Delta^{P}$ and $Q \in \operatorname{int} \Delta^{Q}$, for some simplices $\Delta^{P}, \Delta^{Q}$. Therefore the sets of polytopes of $\operatorname{Star}\left(\Delta^{P} ; \mathbf{K}\right)$ and of $\operatorname{Star}\left(\Delta^{Q} ; \mathbf{K}\right)$ are disjoint. Thus $\mathbf{K}_{(P, Q)}=\mathbf{K}_{(Q, P)}$

Lemma 5.1. Assume $\mathbf{T}_{0}, \mathbf{T}_{1}$ are two el-equivalent triangulations of $\mathbf{K}$. Let $\mathbf{L}$ be any common subcomplex of $\mathbf{T}_{0}$ and $\mathbf{T}_{1}$ which is not changed during the corresponding transformations. Let $P \in|\mathbf{K}|$.

Then $\mathbf{T}_{0(P)}$ and $\mathbf{T}_{1(P)}$ are el-equivalent, and the corresponding transformations do not change the complex $\mathbf{L}_{(P)}$.

Proof. It suffices to consider the case of $\mathbf{T}_{0}, \mathbf{T}_{1}$ that differ by an elementary transformation.

Let $\mathbf{S}^{E}$ denote the elementary subdivision, $\mathbf{C}^{\mathbf{B}}$ the basic complex, $\mathbf{P}^{\mathbf{B}}=$ $\operatorname{conv}\left(A_{0} \ldots A_{n} ; B_{0} \ldots B_{m}\right)$ the basic polytope, and

$$
\mathbf{P}_{h}^{\mathbf{E}}=\operatorname{conv}\left(A_{0} \ldots A_{n} ; B_{0} \ldots B_{m}: C_{1}^{h} \ldots C_{i(h)}^{h}\right),
$$

where $h \in H$, be all the elementary polytopes corresponding to the considered elementary transformation.

By assumption $\mathbf{L}$ is a subcomplex of $\operatorname{Ast}\left(\mathbf{P}^{\mathbf{B}} ; \mathbf{K}\right)$. Consider three cases:

1. $P \notin\left|\mathbf{C}^{\mathbf{B}}\right|$. Then $\mathbf{T}_{0(P)}$ and $\mathbf{T}_{1(P)}$ differ by an elementary transformation on the same basic complex $\mathbf{C}^{B}$. The transformation does not change $\mathbf{L}_{(P)}$.

2. $P \in \mathbf{P}^{\mathbf{B}}$. Then $\mathbf{C}$ is simplicial with respect to $\mathbf{P}^{\mathbf{B}}$. Two canonical triangulations $\mathbf{A}$ and $\mathbf{B}$ of $\mathbf{P}^{\mathbf{B}}$ induce triangulations $\mathbf{T}_{0}, \mathbf{T}_{1}$. By Proposition 3.6, $\mathbf{T}_{0(P)}$ and 
$\mathbf{T}_{1(P)}$ are induced by $\mathbf{A}_{(P)}$ and $\mathbf{B}_{(P)}$. By Proposition 4.B, $\mathbf{A}_{(P)}$ is el-equivalent to $\mathbf{B}_{(P)}$. By Corollary 3.5 and Proposition 3.8 we obtain our assertion: the corresponding transformations only change the triangulations of polytopes which contain $\mathbf{P}^{B}$.

3. $P \notin \mathbf{P}^{\mathbf{B}}$ and $P \in \mathbf{P}^{\mathbf{E}}$ for some $h$. This is the same case as 2 above, using Proposition 4.C instead of Proposition 4.B.

Proposition 5.2. Let $\Delta$ be a simplex and $P_{i} \in \Delta$ for $i=1, \ldots, n$. Assume $P \in$ int $\Delta$. Then

$$
\Delta_{\left(P_{0}, \ldots, P_{j-1}, P, P_{j}, \ldots P_{l}\right)} \text { and } \Delta_{\left(P_{0}, \ldots, P_{k-1}, P, P_{k}, \ldots P_{l}\right)}
$$

are el-equivalent for all $j, k, l$, and the corresponding transformations do not change the relative boundary of $\Delta$.

Proof. It is sufficient to consider the transposition

$$
\Delta_{\left(P_{0} \ldots P_{j-1}, P, P_{j} \ldots P_{l}\right)} \longrightarrow \Delta_{\left(P_{0}, \ldots P_{j}, P, P_{j+1} \ldots P_{l}\right)}
$$

Apply Proposition 4.A to $\Delta_{\left(P_{0} \ldots P_{j-1}\right)}$ and to the points $P_{j}$ and $P$. We get that $\Delta_{\left(P_{0} \ldots P_{j-1}, P, P_{j}\right)}$ and $\Delta_{\left(P_{0} \ldots P_{j-1}, P_{j}, P\right)}$ are el-equivalent and the relevant transformations only change simplices which contain $P$ and $P_{j}$. Therefore none of the simplices lying on the boundary is changed. We then get our statement by applying Lemma 5.1 to the points $P_{j+1}, \ldots, P_{l}$.

\section{Flat Pairs. Equivalence of Blow-Ups of an Arbitrary Complex}

Let $\mathbf{C}$ be a complex in $\mathbf{Q}^{d}$ and $H$ be an affine space. We denote by $\mathbf{C}_{\mid H}$ the complex which consists of all faces of $\mathbf{C}$ contained in $H$. We write $\mathbf{C} \cap H$ for the complex of all the polytopes of the form $\mathbf{P} \cap H$, where $\mathbf{P}$ is a face of $\mathbf{C}$.

Clearly $\mathbf{C}_{\mid H}$ is a subcomplex of $\mathbf{C} \cap H$. Note also that if $\mathbf{C}$ is simplicial then so is $\mathbf{C}_{\mid H}$, and if $\mathbf{C}_{0}$ is a subdivision of $\mathbf{C}_{1}$ then $\mathbf{C}_{0} \cap H$ is also a subdivision of $\mathbf{C}_{1} \cap H$.

Theorem 6.1 ([4], Theorem 1 in 5.1). Every polytope $\mathbf{P}$ is affinely isomorphic to the polytope $\Delta \cap H$, where $\Delta \subset \mathbf{R}^{k}$ is a simplex and $H$ an affine space in $\mathbf{R}^{k}$.

Although this theorem is proved for the field $\mathbf{R}$, it also holds for $\mathbf{Q}$ and the proof is the same.

Proposition 6.2. Let $\mathbf{C}$ be a complex and let $H$ be an affine space. Assume $P \in$ $|\mathbf{C}| \cap H$. Then $\mathbf{C}_{(P)} \cap H=(\mathbf{C} \cap H)_{(P)}$.

Proof. Both operations, blow-up and intersection of complexes, are performed on each polytope separately. Thus it suffices to prove the above for each face $\mathbf{P}$ of $\mathbf{C}$ containing $P$.

Let $\Gamma$ be the set of all faces of $\mathbf{P}$ which do not contain $P$. Let $\Gamma_{H}$ be the of all polytopes $\mathbf{K} \cap H$, where $\mathbf{K}$ is a face of $\mathbf{P}$ and $P \notin \mathbf{K} \cap H$. Clearly $\Gamma_{H}=\{\mathbf{K} \cap H$ : $\mathbf{K} \in \Gamma\}$. Thus

$$
\begin{aligned}
\mathbf{P}_{(P)} & =\{\operatorname{conv}(\mathbf{K} ; P): \mathbf{K} \in \Gamma\} \cup \Gamma \\
\mathbf{P}_{(P)} \cap H & =\{\operatorname{conv}(\mathbf{K} ; P) \cap H: \mathbf{K} \in \Gamma\} \cup\{\mathbf{K} \cap H: \mathbf{K} \in \Gamma\}, \\
& =\{\operatorname{conv}(\mathbf{K} \cap H ; P): \mathbf{K} \in \Gamma\} \cup \Gamma_{H}, \\
& =\left\{\operatorname{conv}(\mathbf{K} ; P): \mathbf{K} \in \Gamma_{H}\right\} \cup \Gamma_{H}=(\mathbf{P} \cap H)_{(P)} .
\end{aligned}
$$


We say that a pair $\left(\Delta ;\left\{P_{i}: i=0, \ldots, l\right\}\right)$ is flat with respect to an affine space $H$ (or $H$-flat) if $\Delta$ is a simplex and $H \cap \Delta=\operatorname{conv}\left(P_{i}: i=0, \ldots, l\right)$ (here the $P_{i}$ are not necessarily vertices)

Proposition 6.3. Let $\mathbf{S}$ be a subdivision of the $H$-flat pair

$$
\left(\Delta ;\left\{P_{i}: i=0, \ldots, l\right\}\right) .
$$

Let $\mathbf{P}=\operatorname{conv}\left(P_{1}, \ldots, P_{l}\right)=\Delta \cap H$. Then $\mathbf{S}_{\mid H}$ is a subdivision of the pair

$$
\left(\mathbf{P} ;\left\{P_{i}: i=0, \ldots, l\right\}\right) \text {. }
$$

Proof. Let $\Delta=\Delta\left(A_{0}, \ldots, A_{k}\right)$ and let $Q \in \mathbf{P}$. By induction on $\operatorname{dim} \Delta$ we show the following fact: For each $Q \in \mathbf{P}$ there exists a polytope $\mathbf{L}^{Q} \in \mathbf{S}$ such that $Q \in \operatorname{int} \mathbf{L}^{Q}$ and $\mathbf{L}^{Q} \subseteq \mathbf{P}$.

This is trivial in dimension one. Now suppose that $\mathbf{L}^{Q} \nsubseteq \mathbf{P}$ for some $Q \in \mathbf{P}$. By assumption $\mathbf{L}^{Q}$ is a polytope of the pair $\left(\Delta ;\left\{P_{i}: i=0, \ldots, l\right\}\right)$.

There are two possible cases.

1. $\mathbf{L}^{Q}=\Delta\left(A_{i(1)}, \ldots, A_{i(n)}\right)$, where $\{i(1), \ldots, i(n)\} \subset\{1, \ldots, k\}$ and $n<k$. Then $L^{Q} \cap H=L^{Q} \cap \mathbf{P}$ is a face of $\mathbf{P}$. Hence $L^{Q} \cap \mathbf{P}=\operatorname{conv}\left\{P_{i}: P_{i} \in\right.$ $\left.\mathbf{L}^{Q}\right\}$. This means that the pair $\left(\mathbf{L}^{Q} ;\left\{P_{i}: P_{i} \in \mathbf{L}^{Q}\right\}\right)$ is flat. By the inductive assumption, $Q \in$ int $\mathbf{L}^{\prime}$ for some polytope $\mathbf{L}^{\prime} \subseteq \mathbf{L}^{Q} \cap \mathbf{P}$ of the pair $\left(\mathbf{L}^{Q} ;\left\{P_{i}: P_{i} \in \mathbf{L}^{Q}\right\}\right)$. This means that $\mathbf{L}^{\prime}$ is also a polytope of the pair $\left(\Delta ;\left\{P_{i}: i=0, \ldots, l\right\}\right)$. Finally, by definition, $\mathbf{L}^{\prime}=\mathbf{L}^{Q} \subseteq \mathbf{P}$.

2. $\mathbf{L}^{Q}=\operatorname{conv}\left(A_{i(1)}, \ldots, A_{i(n)}, P_{j(1)}, \ldots, P_{j(r)}\right)$. Then

$$
Q=a_{i(1)} A_{i(1)}+\ldots+a_{i(n)} A_{i(n)}+p_{j(1)} P_{j(1)}+\ldots+p_{j(r)} P_{j(r)},
$$

where all coefficients are positive.

Since $Q \in H$, then

$$
Q^{\prime}:=\left(a_{i(1)} / \sum a_{i(j)}\right) \cdot A_{i(1)}+\ldots+\left(a_{i(n)} / \sum a_{i(j)}\right) \cdot A_{i(n)} \text { is also in } H \text {. }
$$

But $Q^{\prime} \in \operatorname{int} \Delta\left(A_{i(1)} \ldots A_{i(n)}\right)$, and the simplex $\Delta\left(A_{i(1)} \ldots A_{i(n)}\right)$ is a face of $\mathbf{L}^{Q}$. Now apply 1 to $Q^{\prime}$ and $\Delta\left(A_{i(1)}, \ldots, A_{i(n)}\right)$, to get $\Delta\left(A_{i(1)}, \ldots, A_{i(n)}\right) \subset H$. Finally

$$
L^{Q}=\operatorname{conv}\left(A_{i(1)}, \ldots, A_{i(n)}, P_{j(1)}, \ldots P_{j(r)}\right) \subseteq H .
$$

Corollary 6.4. If $\mathbf{S}$ is a subdivision of an $H$-flat pair $\left(\Delta ;\left\{P_{i} ; i=0, \ldots, 1\right\}\right.$ then $S_{\mid H}=S \cap H$.

Proof. $\mathbf{S}_{\mid H}$ is a subcomplex of $\mathbf{S} \cap H$. On the other hand, their supports are equal to $\left|\mathbf{S}_{\mid H}\right|=|\mathbf{S}| \cap H=|\mathbf{S} \cap H|$.

We say that a blow-up $\mathbf{C}_{\left(P_{1}, \ldots, P_{m}\right)}$ of $\mathbf{C}$ is complete iff $\operatorname{Vert}(\mathbf{C}) \subset\left\{P_{1}, \ldots, P_{m}\right\}$. We say that the blow-up $\mathbf{C}_{\left(P_{1}, \ldots, P_{m}\right)}$ is simplicial if it is a triangulation.

Corollary 6.5. Let $\mathbf{C}_{\left(P_{1}, \ldots, P_{m}\right)}$ be a complete blow-up of $\mathbf{C}$. Then $\mathbf{C}_{\left(P_{1}, \ldots, P_{m}\right)}$ is simplicial.

Proof. It is sufficient to prove the corollary for arbitrary face $\mathbf{P} \in \mathbf{C}$. Then $\mathbf{P}_{\left(P_{1}, \ldots, P_{l}\right)}=\mathbf{P}_{\left(P_{i_{1}}, \ldots, P_{i_{l}}\right)}$, where $P_{i_{1}}, \ldots, P_{i_{n}}$ is the sequence otained from the sequence $\left(P_{1}, \ldots, P_{m}\right)$ by removing all points not belonging to $\mathbf{P}$. By Theorem $6.1 \mathbf{P}=\Delta \cap H$, where $\Delta$ is a simplex and $H$ is some affine space. $\operatorname{Vert}(\mathbf{P}) \subset$ $\left\{P_{i_{1}}, \ldots, P_{i_{l}}\right\}$. Thus the pair $\left(\Delta ;\left\{P_{i_{1}}, \ldots, P_{i_{l}}\right\}\right)$ is $H$-flat, and hence by Corollary 6.4 $\mathbf{P}_{\left(P_{i_{1}}, \ldots, P_{i_{l}}\right)}=\Delta_{\left(P_{i_{1}}, \ldots, P_{i_{l}}\right) \mid H}$ is simplicial. 
Corollary 6.6. Let $\mathbf{P}$ be a polytope in $\mathbf{Q}^{d}$ and $H$ be an affine space in $\mathbf{Q}^{d}$. Let $P_{i} \in \mathbf{P}$ for $i=0, \ldots, n$ be such that $\mathbf{P} \cap H=\operatorname{conv}\left(P_{0}, \ldots, P_{n}\right)$. Then

$$
\mathbf{P}_{\left(P_{0}, \ldots, P_{n}\right) \mid H}=\mathbf{P}_{\left(P_{0}, \ldots, P_{n}\right)} \cap H=(\mathbf{P} \cap H)_{\left(P_{0}, \ldots, P_{n}\right)} .
$$

Proof. The second equality follows from Proposition 6.2. Consider the first equality. By Theorem 6.1 we can assume that $\mathbf{P}=\Delta \cap H^{\prime}$ for some simplex $\Delta$ and affine space $H^{\prime}:=\operatorname{aff}(\mathbf{P})$. By replacing $H$ by $H \cap H^{\prime}$ we can assume that $H \subseteq H^{\prime}$. Then $\mathbf{P} \cap H=\Delta \cap H$, and the pair $\left(\Delta ;\left\{P_{0}, \ldots, P_{n}\right\}\right)$ is $H$-flat. By Proposition 6.2,

$$
\mathbf{P}_{\left(P_{0}, \ldots, P_{n}\right)} \cap H=(\mathbf{P} \cap H)_{\left(P_{0}, \ldots, P_{n}\right)}=(\Delta \cap H)_{\left(P_{0}, \ldots, P_{n}\right)}=\Delta_{\left(P_{0}, \ldots, P_{n}\right)} \cap H .
$$

Since $H \subseteq H^{\prime}$ we infer that $\Delta_{\left(P_{0}, \ldots, P_{n}\right) \mid H}$ is a subcomplex of

$$
\Delta_{\left(P_{0}, \ldots, P_{n}\right)} \cap H_{\mid H}^{\prime}=\mathbf{P}_{\left(P_{0}, \ldots, P_{n}\right) \mid H} .
$$

Since the pair $\left(\Delta ;\left\{P_{0}, \ldots, P_{n}\right\}\right.$ is flat, Corollary 6.4. yields

$$
\Delta_{\left(P_{0}, \ldots, P_{n}\right) \mid H}=\Delta_{\left(P_{0}, \ldots, P_{n}\right)} \cap H .
$$

Finally,

$$
\mathbf{P}_{\left(P_{0}, \ldots, P_{n}\right) \mid H} \supseteq \Delta_{\left(P_{0}, \ldots, P_{n}\right) \mid H}=\Delta_{\left(P_{0}, \ldots, P_{n}\right)} \cap H=\mathbf{P}_{\left(P_{0}, \ldots, P_{n}\right)} \cap H .
$$

The other inclusion is obvious.

Proposition 6.7. Let $\mathbf{T}_{0}, \mathbf{T}_{1}$ be two el-equivalent triangulations of the $H$-flat pair $(\Delta ; \Omega)$. Then $\mathbf{T}_{0 \mid H}, \mathbf{T}_{1 \mid H}$ are el-equivalent triangulations of the polytope $\mathbf{P}:=$ $\Delta \cap H$.

Proof. It is sufficient to prove the proposition for $\mathbf{T}_{0}, \mathbf{T}_{1}$ differing by an elementary transformation.

Let $\mathbf{S}^{\mathbf{E}}$ be the elementary subdivision of that transformation, and $\mathbf{P}^{\mathbf{B}}$ its basic polytope. By Definition 2.3, $\mathbf{S}^{\mathbf{E}}$ consists of simplices and of elementary polytopes belonging to $\operatorname{Star}\left(\mathbf{P}^{\mathbf{B}} ; \mathbf{S}^{\mathbf{E}}\right)$. Consider two cases.

1. $\mathbf{P}^{\mathbf{B}} \subseteq H$. Then $\mathbf{S}_{\mid H}^{\mathbf{E}}$ consists of simplices of $\mathbf{S}^{\mathbf{E}}$ lying in $H$, and of the elementary polytopes of $\operatorname{Star}\left(\mathbf{P}^{\mathbf{B}} ; \mathbf{S}^{\mathbf{E}}\right)$ lying in $H$. That means that $\mathbf{S}^{\mathbf{E}}{ }_{\mid H}$ consists of simplices and elementary polytopes belonging to $\operatorname{Star}\left(\mathbf{P}^{\mathbf{B}} ; \mathbf{S}_{\mid H}^{\mathbf{E}}\right)$, and thus $\mathbf{S}_{\mid H}^{\mathbf{E}_{\mid H}}$ is an elementary subdivision. By Proposition $6.3, \mathbf{P}=\left|\mathbf{T}_{0 \mid H}\right|=\mid$ $\mathbf{T}_{1 \mid H}|=| \mathbf{S}_{\mid H}^{E} \mid$. By Corollary 6.4, $\mathbf{T}_{i \mid H}=\mathbf{T}_{i} \cap H$ for $i=0,1$ are subdivisions of $\mathbf{S}_{\mid H}^{E}=\mathbf{S}^{E} \cap H$. Again by Proposition $6.3, \operatorname{Vert}\left(\mathbf{T}_{i \mid H}\right)=\operatorname{Vert}\left(\mathbf{S}_{\mid H}^{E}\right)=\Omega$. Finally $\mathbf{T}_{0 \mid H}, \mathbf{T}_{1 \mid H}$ are two different proper triangulations of $\mathbf{S}_{\mid H}^{\mathbf{E}}$, which means that they differ by an elementary transformation.

2. $\mathbf{P}^{\mathbf{E}}$ is not contained in $H$. Then none of the elementary polytopes of $\mathbf{S}^{\mathbf{E}}$ lies in $H$. Therefore $\mathbf{S}_{\mid H}^{\mathbf{E}}$ consists only of simplices. Hence $\mathbf{T}_{0 \mid H}, \mathbf{T}_{1 \mid H}$ are equal, as they are proper triangulations of $\mathbf{S}_{\mid H}$.

Proposition 6.8. Let $\mathbf{C}_{\left(P_{1}, \ldots, P_{m}\right)}$ be a complete blow-up of a complex $\mathbf{C}$. Let $\operatorname{dim} \mathbf{C}=n$. Let $P_{i(j)}, j=1, \ldots, l$, be the sequence obtained from $P_{1}, \ldots, P_{m}$ by omitting all points belonging to the relative interior of the $n$-dimensional polytopes of $\mathbf{C}$. Then $\left.\mathbf{C}_{\left(P_{i(1)}, \ldots, P_{i(l)}\right.}\right)$ is also a complete blow-up of $\mathbf{C}$, and moreover $\mathbf{C}_{\left(P_{i(1)}, \ldots, P_{i(l)}\right)}$ is equivalent to $\mathbf{C}_{\left(P_{1}, \ldots, P_{m}\right)}$. 
Proof. Let $P_{p(j)}, j=1, \ldots, m-l$, denote the interior points of $n$-dimensional polytopes in the sequence $P_{1}, \ldots, P_{m}$ taken in the same order. We show first that the triangulations $\mathbf{C}_{\left(P_{i(1)}, \ldots, P_{i(l)}, P_{p(1)}, \ldots, P_{p(m-l)}\right)}$ and $\mathbf{C}_{\left(P_{1}, \ldots, P_{m}\right)}$ are el-equivalent.

It suffices to show that $\mathbf{C}_{\left(*, P_{i(h)}, P_{p(g)}, * *\right)}$ and $\mathbf{C}_{\left(*, P_{p(g)}, P_{i(h)}, * *\right)}$ are el-equivalent for each $h$ and $g$ and arbitrary * and **.

If $P_{i(h)}, P_{p(g)}$ do not belong to any common $n$-dimensional polytope then both triangulations coincide. Assume that $P_{i(h)}, P_{p(g)}$ belong to a common $n$-dimensional polytope $\mathbf{P}$. Then the given blow-ups are the same outside $\mathbf{P}$.

Consider the polytope $\mathbf{P}$. By Theorem 6.1 we can write $\mathbf{P}=\Delta \cap H$, where $\Delta$ is a simplex and $H$ is an affine space. By assumption of completeness of the blow-up $\mathbf{C}_{\left(P_{1}, \ldots, P_{m}\right)}$ we have that $\operatorname{vert}(\mathbf{P}) \subset\left\{P_{1}, \ldots, P_{m}\right\}$. Thus the pair $\left(\Delta ;\left\{P_{1}, \ldots, P_{m}\right\} \cap \mathbf{P}\right)$ is flat. Remove from the sequences $\left(*, P_{i(h)}, P_{p(g)}, * *\right)$ and $\left(*, P_{p(g)}, P_{i(h)} * *\right)$ all points which are not in $\mathbf{P}$. Then we get two permutations of the set $\left\{P_{1}, \ldots, P_{m}\right\} \cap \mathbf{P}:\left(*^{\prime}, P_{i(h)}, P_{p(g)}, * *^{\prime}\right)$ and $\left(*^{\prime}, P_{p(g)}, P_{i(h)} * *^{\prime}\right)$ for the respective $*^{\prime}$ and $* *^{\prime}$. By Proposition 5.2, $\Delta_{\left(*^{\prime}, P_{i(h)}, P_{p(g)}, * *^{\prime}\right)}$ and $\Delta_{\left(*^{\prime}, P_{p(g)}, P_{i(h)}, * *^{\prime}\right)}$ are el-equivalent and the corresponding transformations do not change them on the boundary of $\Delta$. Hence by Corollary 6.6 and Proposition 6.7

$$
\Delta_{\left(*^{\prime}, P_{i(h)}, P_{p(g)}, * *^{\prime}\right) \mid H}=\mathbf{P}_{\left(*^{\prime}, P_{i(h)}, P_{p(g)}, * *^{\prime}\right)}=\mathbf{P}_{\left(*, P_{i(h)}, P_{p(g)}, * *\right)}
$$

is el-equivalent to

$$
\Delta_{\left(*^{\prime}, P_{p(g)}, P_{i(h)}, * *^{\prime}\right) \mid H}=\mathbf{P}_{\left(*^{\prime}, P_{p(g)}, P_{i(h)}, * *^{\prime}\right)}=\mathbf{P}_{\left(*, P_{p(g)}, P_{i(h)}, * *\right)} .
$$

Moreover the corresponding transformations do not change the triangulations on the boundary of $\mathbf{P}$. Since the triangulations $\mathbf{C}_{\left(*, P_{i(h)}, P_{p(g)}, * *\right)}$ and $\mathbf{C}_{\left(*, P_{p(g)}, P_{i(h)}, * *\right)}$ are the same outside of $\mathbf{P}$, the el-equivalence on $\mathbf{P}$ extends to the el-equivalence of these two triangulations. Thus $\mathbf{C}_{\left(P_{i(1)}, \ldots, P_{i(l)}, P_{p(1)}, \ldots, P_{p(m-l)}\right)}$ and $\mathbf{C}_{\left(P_{1}, \ldots, P_{m}\right)}$ are el-equivalent. But in the first triangulation we can blow down the points $P_{p(i))}$ for $i=1, \ldots, m-l$ in the opposite order to get the triangulation $\mathbf{C}_{\left(P_{i(1)}, \ldots, P_{i(l)}\right)}$. Note that all these blow-downs are done in the category of simplicial complexes because we here operate with complete blow-ups of $\mathbf{C}$ (Corollary 6.5).

Proposition 6.9. Any two simplicial blow-ups of an arbitrary complex $\mathbf{C}$ are equivalent.

Proof. We can assume that the considered blow-ups are complete and have the same set of vertices. (If this is not the case we blow-up the triangulations at the missing points, which does not change the equivalence class). This means that the triangulations are of the form

$$
\mathbf{C}_{\left(Q_{\sigma_{0}(1)}, \ldots, Q_{\sigma_{0}(m)}\right)} \text { and } \quad \mathbf{C}_{\left(Q_{\sigma_{1}(1)}, \ldots, Q_{\sigma_{1}(m)}\right)},
$$

where $\sigma_{0}, \sigma_{1}$ are two permutations of the set $\{1, \ldots, m\}$ and $\left\{Q_{i}: i=1, \ldots, m\right\} \supseteq$ $\operatorname{Vert}(\mathbf{C})$.

We prove our proposition by induction on $\operatorname{dim} \mathbf{C}$. For $n=0$ the proposition is obvious. Assume it holds for all $k$-dimensional complexes where $k<n$, and let $\operatorname{dim} \mathbf{C}=n$. Then $\mathbf{C}$ has a finite number of $n$-dimensional polytopes, say $\mathbf{P}_{j}$, for $j=1, \ldots, w$. In each $\mathbf{P}_{j}$ we choose a point $P_{j} \in \operatorname{int} \mathbf{P}_{j}$.

For any permutation $\sigma$ of the set $\{1, \ldots, m\}$, by $\left(Q_{\sigma(i(s))} ; s=1, \ldots, h\right)$ where $h \leq m$ we mean the sequence obtained from the sequence $Q_{\sigma(i)}$ by omitting interior points of $n$-dimensional faces of $\mathbf{C}$. Then by Proposition 6.8 the 
complete blow-up $\mathbf{C}_{\left(Q_{\sigma(i(1))}, \ldots, Q_{\sigma(i(h))}\right)}$ is equivalent to both $\mathbf{C}_{\left(Q_{\sigma(1)}, \ldots, Q_{\sigma(m)}\right)}$ and $\mathbf{C}_{\left(P_{1}, \ldots, P_{w}, Q_{\sigma(i(1))}, \ldots, Q_{\sigma(i(h))}\right)}$. Thus we can we reduce the equivalence of

$$
\mathbf{C}_{\left(Q_{\sigma_{0}(1)}, \ldots, Q_{\sigma_{0}(m)}\right)} \quad \text { and } \quad \mathbf{C}_{\left(Q_{\sigma_{1}(1)}, \ldots, Q_{\sigma_{1}(m)}\right)}
$$

to the equivalence of

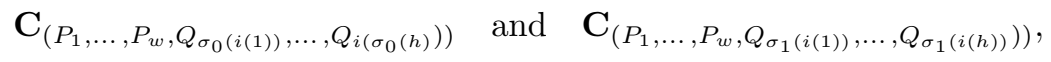

hence to the equivalence of the complete blow-ups of the complex $\mathbf{C}_{\left(P_{1}, \ldots, P_{w}\right)}$ with centers lying in the $(n-1)$-dimensional skeleton $\mathbf{C}(n-1)$ of $\mathbf{C}$.

Note that the complex $\mathbf{C}_{\left(P_{1}, \ldots, P_{w}\right)}$ is simplicial with respect to its subcomplex $\mathbf{C}(n-1)$. By the inductive assumption the blow-ups

$$
\mathbf{C}(n-1)_{\left(Q_{\sigma_{0}(i(1))} \ldots Q_{\sigma_{0}(i(h))}\right)} \text { and } \mathbf{C}(n-1)_{\left(Q_{\sigma_{1}(i(1))} \ldots Q_{\sigma_{1}(1(h))}\right)}
$$

are equivalent. By Propositon 3.6 we have:

$$
\begin{aligned}
& \mathbf{C}_{\left(P_{1}, \ldots, P_{w}\right)}\left(\mathbf{C}(n-1)_{\left(Q_{\sigma_{0}(i(1))} \ldots Q_{\sigma_{0}(i(h))}\right)}=\mathbf{C}_{\left(P_{1}, \ldots, P_{w}, Q_{\sigma_{0}(i(1))} \ldots Q_{\sigma_{0}(i(h))}\right)},\right. \\
& \mathbf{C}_{\left(P_{1}, \ldots, P_{w}\right)}\left(\mathbf{C}(n-1)_{\left(Q_{\sigma_{1}(i(1))} \ldots Q_{\sigma_{1}(1(h))}\right)}=\mathbf{C}_{\left(P_{1}, \ldots, P_{w}, Q_{\sigma_{1}(i(1))} \ldots Q_{\sigma_{1}(i(h))}\right)} .\right.
\end{aligned}
$$

By Corollary 3.5 and the above, the two triangulations are equivalent.

\section{Flat Cuts. Equivalence of All Triangulations of a Complex}

Proposition 6.9 allows us to extend the equivalence of triangulations to equivalence of subdivisions: We say that two subdivisions $\mathbf{S}_{0}, \mathbf{S}_{1}$ of a given complex $\mathbf{C}$ are equivalent if some of their simplicial blow-ups are equivalent.

Definition 7.1. Let $\mathbf{C}$ be a complex in $\mathbf{Q}^{d}$. Let $\mathbf{S}$ be any subdivision of $\mathbf{C}$, and $H$ be a hyperplane in $\mathbf{Q}^{d}$. The flat cut $\mathbf{S} \cdot H$ of $\mathbf{S}$ with $H$ is a subdivision $\mathbf{S}^{\prime}$ such that every polytope of it is the intersection of some polytope of $\mathbf{S}$ with $H$, or with one of the halfspaces determined by $H$.

Proposition 7.2. Let $\mathbf{S}$ be any subdivision of a complex $\mathbf{C}$. Then $\mathbf{S} \cdot H$ is equivalent to $\mathbf{S}$.

Proof. Let $\left\{P_{1}, \ldots, P_{k}\right\}=\operatorname{Vert}(\mathbf{S} \cap H)$. We have to show that there exist simplicial blow-ups of $\mathbf{S}$ and of $\mathbf{S} \cdot H$ which are equivalent. We will in fact show that $\mathbf{S}_{\left(P_{1}, \ldots, P_{k}\right)}=(\mathbf{S} \cdot H)_{\left(P_{1}, \ldots, P_{k}\right)}$ is their common blow-up, which can be further blown up to a common simplicial blow-up. By arguments similar to those in Proposition 6.3 we see that $(\mathbf{S} \cdot H)_{\left(P_{1}, \ldots, P_{k}\right)}=\left(\mathbf{S}_{\left(P_{1}, \ldots, P_{k}\right)}\right) \cdot H$ It is sufficient to show that $\left(\mathbf{S}_{\left(P_{1}, \ldots, P_{k}\right)}\right) \cdot H=\mathbf{S}_{\left(P_{1}, \ldots, P_{k}\right)}$. We prove this for an arbitrary face $\mathbf{P}$ of $\mathbf{S}$. Let $\left\{P_{i(j)}: j=1, \ldots, n\right\}$ be those $P_{i}$ which lie in $\mathbf{P}$. Then $\mathbf{P}_{\left(P_{1}, \ldots, P_{k}\right)}=\mathbf{P}_{\left(P_{i(1)}, \ldots, P_{i(n)}\right)}$. From Corollary 6.6 we get

$$
\mathbf{P}_{\left(P_{i(1)}, \ldots, P_{i(n)}\right)} \cap H=\mathbf{P}_{\left(P_{i(1)}, \ldots, P_{i(n)}\right) \mid H}=\mathbf{P}_{\left(P_{1}, \ldots, P_{k}\right) \mid H} .
$$

That means that intersection with $H$ of every polytope $\mathbf{P}^{\prime}$ of $\mathbf{P}_{\left(P_{1}, \ldots, P_{k}\right)}$ is a face of $\mathbf{P}^{\prime}$. Hence this polytope is contained either in $H$ or in one of the halfspaces determined by $H$. Thus $\mathbf{P}_{\left(P_{1}, \ldots, P_{k}\right)}=\mathbf{P}_{\left(P_{1}, \ldots, P_{k}\right)} \cdot H$ because we get no new polytopes.

Proposition 7.3. Let $\mathbf{C}$ be a complex in $\mathbf{Q}^{m}$. Then all triangulations of $\mathbf{C}$ are equivalent. 
Proof. Let $\mathbf{T}_{1}$ and $\mathbf{T}_{2}$ be any two triangulations. Every polytope of $\mathbf{T}_{1}$ and $\mathbf{T}_{2}$ is the intersection of a face of $\mathbf{C}$ with some halfspaces in $\mathbf{Q}^{m}$. Take hyperplanes $H_{i}$ for $i=1, \ldots, n$ determining all the halfspaces associated with all the faces of $\mathbf{T}_{0}$ and $\mathbf{T}_{1}$. We get that

$$
\mathbf{T}_{0} \cdot H \cdot \ldots \cdot H_{n}=\mathbf{T}_{1} \cdot H_{1} \cdot \ldots \cdot H_{n} .
$$

By the previous lemma we are done.

\section{Simplicial Case of Theorem A}

Consider a simplicial fan $\Sigma$ in $\mathbf{Q}^{n}$. Let $\Sigma(1)=\left\{\rho_{i}: i \in I\right\}$ denote the set of edges of the fan $\Sigma$. Attach to each edge $\rho_{i} \in \Sigma(1)$ any nonzero vector $v_{i} \in \rho_{i}$. It will determine the correspondence between cones $\left\langle v_{i_{1}}, \ldots, v_{i_{j}}\right\rangle \in \Sigma$ and simplices $\operatorname{conv}\left(\rho_{i_{1}}, \ldots, \rho_{i_{j}}\right)$, which gives rise to a correspondence of fan $\Sigma$ and the complex

$$
\mathbf{C}(\Sigma):=\left\{\operatorname{conv}\left(\rho_{i_{1}}, \ldots, \rho_{i_{j}}\right):\left\langle v_{i_{1}}, \ldots, v_{i_{j}}\right\rangle\right\}
$$

Any decomposition $\Sigma^{\prime}$ of $\Sigma$ determines a subdivision $\Sigma^{\prime} \cap \mathbf{C}(\Sigma)$ of $\mathbf{C}(\Sigma)$ and vice versa. Thus we have a natural correspondence $f_{\Sigma}$ between decompositions (resp. simplicial decompositions of $\Sigma$ ) and subdivisions and triangulations of the complex $\mathbf{C}(\Sigma)$. The above correspondence is a bijection. The inverse map is given as follows: With a subdivision $\mathbf{S}$ of $\mathbf{C}(\Sigma)$ we associate a fan $\left\{\sigma_{i}=\operatorname{conv}\left(t \cdot \Delta_{i}\right)\right.$ : where $\left.\Delta_{i} \in \mathbf{S} ; t \geq 0\right)$. The blow-ups and blow-downs of fans are in 1-1 correspondence with the blow-ups and blow-downs of the respective complexes.

Propositions 7.3 can be translated into the following theorem:

Theorem 8.1. Let $\mathbf{C}$ be an arbitrary complex in $\mathbf{Q}^{m}$. Let $\mathbf{T}^{\prime}, \mathbf{T}^{\prime \prime}$ be triangulations of $\mathbf{C}$. Then there exists a sequence of triangulations $\mathbf{T}_{i}$ of $\mathbf{C}$ for $i=0, \ldots, n$ such that $\mathbf{T}_{0}=\mathbf{T}^{\prime}, \mathbf{T}_{n}=\mathbf{T}^{\prime \prime}$ and each $\mathbf{T}_{i+1}$ is obtained from $\mathbf{T}_{i}$ by a blow-up or blowdown.

Note that the above theorem is valid also over the field of real numbers.

Let $\Sigma$ be a fan in $\mathbf{Q}^{n}$. By means of the correspondence $f_{\Sigma}$ we can translate the above theorem into a theorem on fans:

Theorem 8.2. Let $\Sigma$ be a simplicial fan in $\mathbf{Q}^{m}$. Let $\Sigma^{\prime}, \Sigma^{\prime \prime}$ be simplicial decompositions of $\Sigma$. Then there exists a sequence of simplicial decompositions $\Sigma_{i}$ of $\Sigma$ for $i=0, \ldots, n$ such that, $\Sigma_{0}=\Sigma^{\prime}, \Sigma_{n}=\Sigma^{\prime \prime}$, and each $\Sigma_{i+1}$ is obtained from $\Sigma_{i}$ by a blow-up or blow-down.

Corollary 8.3. Let $\Sigma^{\prime}$, $\Sigma^{\prime \prime}$ be two simplicial fans such that $\left|\Sigma^{\prime}\right|=\left|\Sigma^{\prime \prime}\right|$. Then there exists a sequence of simplicial fans $\Sigma_{i}$, for $i=0, \ldots, n$, such that $\Sigma_{0}=\Sigma^{\prime}$, $\Sigma_{n}=\Sigma^{\prime \prime}$, and each $\Sigma_{i+1}$ is obtained from $\Sigma_{i}$ by a blow-up or blow-down.

Proof. For any two fans $\Sigma^{\prime}, \Sigma^{\prime \prime}$ we can easily find a common simplicial subdivision $\Sigma$. Then we apply Theorem 8.2. to $\Sigma^{\prime}$ and $\Sigma$, and to $\Sigma$ and $\Sigma^{\prime \prime}$.

\section{PART 3}

Let $\Sigma$ be a simplicial fan in $\mathbf{Q}^{n+1}$. In the previous section we attached to it the complex $\mathbf{C}(\Sigma) \subset \mathbf{Q}^{n+1}$. Now we shall introduce on $\mathbf{C}(\Sigma)$ an integral structure, which will correspond to the lattice structure. Namely on the set $|\mathbf{C}(\Sigma)|$ we have a function $[\cdot]: \mathbf{C}(\Sigma) \rightarrow \mathbf{Q}^{n+1}$ which associates with $P \in|\mathbf{C}(\Sigma)|$ the only primitive vector $[\mathrm{P}]$ lying on the ray $t \cdot P$, where $t \geq 0$, and $t \in \mathbf{Q}$. By definition this function 
is an injection. We shall call the complex $\mathbf{C}(\Sigma)$ equipped in such a function a Farey complex (or F-complex). (See [10].) Using this function allows us to talk of regular triangulations, regular blow-ups, determinants, etc. of simplicial complexes. Namely, a triangulation $\mathbf{T}$ of $\mathbf{C}(\Sigma)$ is regular if coresponding decomposition of $\Sigma$ is regular. A blow-up of a triangulation is regular if the corresponding blow-up is regular, etc.

\section{BAsic Definitions}

Let $V=\mathbf{Q}^{k} \supset \mathbf{Z}^{k}$. Let $V^{\prime}$ be an $l$-dimensional vector subspace of $V$ with a fixed orientation. Then $V^{\prime}$ contains the $l$-dimensional lattice $L=\mathbf{Z}^{k} \cap V^{\prime}$.

For vectors $v_{1}, \ldots, v_{k}$, in $L$, by $\operatorname{det}\left(v_{1}, \ldots, v_{k}\right)$ we mean the determinant of the matrix $\left(v_{1}, \ldots, v_{k}\right)$, where all $v_{i}$ are taken in a standard basis of $L$.

Let $\mathbf{C}$ be a $F$-complex in $\mathbf{Q}^{k}$. Let $B_{1}, \ldots, B_{l}$ belong to a face of $\mathbf{C}$. Fix an oriented $(l-1)$-dimensional affine subspace $H$ of $\mathbf{Q}^{k}$, containing $B_{1}, \ldots, B_{l}$. Then $V^{\prime}=\operatorname{aff}(H, 0)$ is an oriented vector subspace of $\mathbf{Q}^{k}$. Define

$$
\operatorname{det}\left(B_{1}, \ldots, B_{l}\right):=\operatorname{det}\left(\left[B_{1}\right], \ldots,\left[B_{l}\right]\right) .
$$

Let $\Delta=\Delta\left(B_{1}, \ldots, B_{l}\right)$ be a simplex contained in a face of $\mathbf{C}$. Let

$$
H=\operatorname{aff}\left(B_{1}, \ldots, B_{l}\right) .
$$

Choose an orientation of $H$. Define

$$
\operatorname{Det} \Delta:=\left|\operatorname{det}\left(B_{1}, \ldots, B_{l}\right)\right|
$$

Lemma 9.1 ([1, Proposition 8.2]). If a simplex $\Delta=\Delta\left(B_{0}, \ldots, B_{l}\right)$ is not regular there is a point $A \in \Delta$ such that $\operatorname{Det} \Delta\left(A, B_{0}, \ldots, \check{B}_{i}, \ldots, B_{l}\right)<\operatorname{Det} \Delta$ for all $i$.

Lemma 9.2. Let $\Delta=\Delta\left(B_{0}, \ldots, B_{l}, C_{1}, \ldots, C_{m}\right)$. Set $H=\operatorname{aff}\left(B_{0}, \ldots, B_{l}\right)$. Then Det $\Delta=\operatorname{Det} \Delta\left(B_{0}, \ldots, B_{l}\right) \cdot f\left(C_{1}, \ldots, C_{m} ; H\right)$, where $f\left(C_{1}, \ldots, C_{m} ; H\right)$ is an integer which depends only on $C_{1}, \ldots, C_{m}, H$

Proof. Let $v_{0}, \ldots, v_{l}$ be a basis of the lattice $($ aff $\left.H, 0)\right) \cap \mathbf{Z}^{k+1}$. Then

$$
\operatorname{det}\left(B_{0}, \ldots, B_{l}, C_{1}, \ldots, C_{m}\right)=\operatorname{det}\left(B_{0}, \ldots, B_{l}\right) \cdot \operatorname{det}\left(v_{0}, \ldots v_{l},\left[C_{1}\right], \ldots,\left[C_{m}\right]\right)
$$

Set $f\left(C_{1}, \ldots, C_{m} ; H\right)=\left|\operatorname{det}\left(v_{0}, \ldots, v_{l},\left[C_{1}\right], \ldots,\left[C_{m}\right]\right)\right|$.

Let $\mathbf{K}$ be an $F$-complex and $\Delta$ be a face. The determinant $\operatorname{Det}(\operatorname{Star}(\Delta ; \mathbf{K}))$ of $\operatorname{Star}(\Delta ; \mathbf{K})$ is the maximum of determinants of the simplices in the star.

By the multiplicative factor $\mu(\operatorname{Star}(\Delta ; \mathbf{K}))$ of $\operatorname{Star}(\Delta ; \mathbf{K})$, we mean

$$
\mu(\operatorname{Star}(\Delta ; \mathbf{K}))=\operatorname{Det}(\operatorname{Star}(\Delta)) / \operatorname{Det} \Delta
$$

which by 9.2 is a positive integer.

Proposition-Definition 9.3. Let $\mathbf{K}$ be a complex, simplicial w.r.t a face $\mathbf{P}$. Let $\mathbf{T}^{\prime}$ be a triangulation of $\mathbf{P}$ and $\Delta \in \mathbf{T}_{\text {max }}^{\prime}$. Then $\mu\left(\operatorname{Star}\left(\Delta^{\prime} ; \mathbf{K}\left(\mathbf{T}^{\prime}\right)\right)\right.$ is independent of $\Delta^{\prime}$ and $\mathbf{T}^{\prime}$.

We shall call this number the multiplicative factor of $\operatorname{Star}(\mathbf{P}, \mathbf{K})$ and denote it by $\mu(\operatorname{Star}(\mathbf{P} ; \mathbf{K}))$ 
Proof. Let $\mathbf{P}=\operatorname{conv}\left(A_{0}, \ldots, A_{l}\right)$. Then

$$
\operatorname{Star}(\mathbf{P}, \mathbf{K})_{\max }=\left\{\operatorname{conv}\left(A_{0}, \ldots, A_{l}, B_{1}^{i}, \ldots, B_{s(i)}^{i}\right), i=1, \ldots, r\right\} .
$$

By assumption $\left(B_{1}^{i}, \ldots, B_{s(i)}^{i}\right)$ are affinely independent of the points from the affine space $H:=\operatorname{aff}\left(A_{0}, \ldots, A_{l}\right)$ (see definition in Section 3 ).

For $\Delta^{\prime}=\Delta\left(A_{0}^{\prime}, \ldots, A_{n}^{\prime}\right)$ we have that

$$
\operatorname{Star}\left(\Delta^{\prime} ; \mathbf{T}\right)_{\max }=\left\{\Delta\left(A_{0}^{\prime}, \ldots, A_{n}^{\prime}, B_{1}^{i}, \ldots, B_{s(i)}^{i}\right), i=1, \ldots, r\right\} .
$$

By Lemma 9.2

$\operatorname{Det} \Delta\left(A_{0}^{\prime}, \ldots, A_{n}^{\prime}, B_{1}^{i}, \ldots, B_{s(i)}^{i}\right)=\operatorname{Det} \Delta\left(A_{0}^{\prime}, \cdots, A_{n}^{\prime}\right) \cdot f\left(B_{1}^{i}, \ldots, B_{s(i)}^{i} ; H\right)$.

Finally, $\mu\left(\operatorname{Star}\left(\Delta^{\prime}, T\right)=\max \left\{f\left(B_{1}^{i}, \ldots, B_{s(i)}^{i} ; H\right)\right\}\right.$.

Fix a $\mathbf{K}$ and $n \in \mathbf{Z}_{\geq 0}$. An (n)-blow-up of $\mathbf{K}$ is a blow-up $\mathbf{K}^{\prime}$ such that all simplices of $\mathbf{K}^{\prime}$ which are not simplices of $\mathbf{K}$ have determinant $\leq n$. In particular:

Lemma 9.4. A (1)-blow-up of a regular complex is a regular blow-up.

Corollary 9.5. Let $\mathbf{K}$ be a complex simplicial w.r.t a face $\mathbf{P}$. Let $\mathbf{T}$ be a triangulation of $\mathbf{P}$. Let $Q \in \operatorname{int} P$ determines an $(n)$-blow-up of $\mathbf{T}$. Then $Q$ determines a $(\mu(\operatorname{Star}(\mathbf{P}, \mathbf{K}) \cdot n))$-blow-up of $\mathbf{K}(T)$.

Let $\mathbf{T}_{0}$ and $\mathbf{T}_{1}$ be triangulations of a complex $\mathbf{K}$ that differ by an elementary transformation at a basic complex $\mathbf{C}^{\mathbf{B}}$. Assume the determinants of all simplices of $\mathbf{T}_{0 \mid \mathbf{C}^{\mathrm{B}}}$ are $\leq r$ and the determinants of all simplices of $\mathbf{T}_{1 \mid \mathbf{C}^{\mathrm{B}}}$ are $\leq s$. Then we say that $\mathbf{T}_{0}$ and $\mathbf{T}_{1}$ differ by an elementary transformation of type $(r, s)$ (if $r \geq s$ ) or $(s, r)$ (if $s \geq r$ ).

Corollary 9.6. Let $\mathbf{K}$ be a complex simplicial w.r.t. a face $\mathbf{P}$. Let $\mathbf{T}_{0}$ and $\mathbf{T}_{1}$ be two triangulations of $\mathbf{P}$ which differ by elementary transformations of type $(r, s)$. Assume that these transformations do not change triangulations on the boundary of $P$. Then $\mathbf{K}\left(\mathbf{T}_{0}\right)$ and $\mathbf{K}\left(\mathbf{T}_{1}\right)$ differ by elementary transformations of type $\mu(\operatorname{Star}(\mathbf{P} ; \mathbf{K})) \cdot r$ and $\mu(\operatorname{Star}(\mathbf{P} ; \mathbf{K})) \cdot s$

A decreasing blow-up of an $F$-complex $\mathbf{K}$ is a blow-up such that the determinant of every simplex which is divided is greater than the determinants of the simplices obtained by its subdivision.

By Lemma 9.1, every non-regular simplex or $F$-complex admits a decreasing blow-up. Note also that decreasing blow-ups do not change any regular simplices.

Lemma 9.7. Let $\mathbf{K}$ be an $F$-complex and $P \in|\mathbf{K}|$. Assume that $P \in \Delta$ for $\Delta \in \mathbf{K}$. Then the blow-up of $\mathbf{K}$ at $P$ is decreasing iff it is decreasing for $\Delta$.

A multiple decreasing blow-up of a given triangulation $\mathbf{T}$ is a triangulation obtained by a composition of finitely many (perhaps zero) decreasing blow-ups of $\mathbf{T}$. By definition a multiple decreasing blow-up does not change any regular simplices.

With a triangulation $\mathbf{T}$ of $\mathbf{K}$ we associate the word

$$
\omega(\mathbf{T}):=\left(\omega_{1}(\mathbf{T}), \ldots, \omega_{i}(\mathbf{T}), \ldots\right)
$$

where $\omega_{i}(\mathbf{T})$ is the number of maximal faces of $\mathbf{K}$ with determinant $i$. In particular $\omega_{i}(\mathbf{T})=0$ for $i>>0$. Define

$$
\omega^{j}(\mathbf{T})=\left(\omega_{j}(\mathbf{T}), \omega_{j+1}(\mathbf{T}), \ldots\right) .
$$


Let $\mathbf{T}_{1}$ and $\mathbf{T}_{2}$ be triangulations of $\mathbf{K}$. Define $\omega\left(\mathbf{T}_{1}\right)>\omega\left(\mathbf{T}_{2}\right)$ if there exists $j \in \mathbf{N}$ such that $\omega_{i}\left(\mathbf{T}_{1}\right)=\omega_{i}\left(\mathbf{T}_{2}\right)$ if $i>j$ and $\omega_{j}\left(\mathbf{T}_{1}\right)>\omega_{j}\left(\mathbf{T}_{2}\right)$.

Analogously we define $\omega\left(\mathbf{T}_{1}\right) \geq \omega\left(\mathbf{T}_{2}\right), \omega^{r}\left(\mathbf{T}_{1}\right)>\omega^{r}\left(\mathbf{T}_{2}\right), \omega^{r}\left(\mathbf{T}_{1}\right) \geq \omega^{r}\left(\mathbf{T}_{2}\right)$ for some $r>0$

Lemma 9.8. If $\omega^{r}\left(\mathbf{T}_{1}\right) \leq \omega^{r}\left(\mathbf{T}_{2}\right)$ and $s \geq r$ then $\omega^{s}\left(\mathbf{T}_{1}\right) \leq \omega^{s}\left(\mathbf{T}_{2}\right)$.

Lemma 9.9. If $\mathbf{T}_{1}$ is a decreasing blow-up of $\mathbf{T}_{2}$ then $\omega\left(\mathbf{T}_{1}\right)<\omega\left(\mathbf{T}_{2}\right)$.

Lemma 9.10. If $\mathbf{T}_{1}$ is an (n)-blow-up of $\mathbf{T}_{2}$ then $\omega_{n+1}\left(\mathbf{T}_{1}\right) \leq \omega_{n+1}\left(\mathbf{T}_{2}\right)$.

Lemma 9.11. If $\mathbf{T}_{1}$ and $\mathbf{T}_{2}$ differ by elementary transformation of type $(n, n)$ then $\omega_{n+1}\left(\mathbf{T}_{1}\right)=\omega_{n+1}\left(\mathbf{T}_{2}\right)$.

A regular decreasing decomposition of an $F$-complex $\mathbf{K}$ (or simply a decreasing decomposition of $\mathbf{K}$ ) is a multiple decreasing blow-up which is a regular complex.

Lemma 9.12. Each $F$-complex $\mathbf{K}$ admits a decreasing decomposition.

Proof. By Lemma 9.9 each decreasing blow-up of $\mathbf{T}$ decreases $\omega(\mathbf{T})$. But we cannot decrease this number infinitely many times.

We call a triangulation good iff any two decreasing decompositions of it can be connected by a finite sequence of regular blow-downs and regular blow-ups. In particular every regular triangulation is good, since it has a unique trivial decreasing decomposition. We say that two good triangulations $\mathbf{T}_{0}$ and $\mathbf{T}_{1}$ are regular equivalent iff some of their decreasing decompositions can be connected by a finite sequence of regular blow-ups and regular blow-downs. We show in the next sections that all good, and in particular regular, triangulations of a given $F$-complex $\mathbf{C}$ are regular equivalent.

Lemma 9.13. A multiple decreasing blow-up of a good triangulation is a good triangulation regular equivalent to the original one.

Proof. This follows from the fact that each decreasing decomposition of a multiple decreasing blow-up of the triangulation is in fact the decreasing decomposition of that triangulation.

\section{Relations Between Determinants of Simplices in a Basic Polytope}

Lemma 10. Let $\mathbf{P}^{\mathrm{B}}:=\operatorname{conv}\left(A_{0}, \ldots, A_{n} ; B_{0}, \ldots, B_{m}\right)$ be a basic polytope contained in a face of an F-complex $\mathbf{C}$ in $\mathbf{Q}^{k}$. Fix an orientation of the affine space $H=\operatorname{aff}\left(A_{0}, \ldots, A_{n}, B_{0}, \ldots, B_{m}\right)$. Then,

$$
\begin{aligned}
& \sum(-1)^{i} \operatorname{det}\left(A_{0}, \ldots, \check{A}_{i}, \ldots, A_{n}, B_{0}, \ldots, B_{m}\right)\left[A_{i}\right] \\
& \quad=\sum(-1)^{n+j} \operatorname{det}\left(A_{0}, \ldots, A_{n}, B_{0}, \ldots, \check{B}_{j}, \ldots, B_{m}\right)\left[B_{j}\right]=g[P],
\end{aligned}
$$

where $P=\Delta\left(A_{0}, \ldots, A_{n}\right) \cap \Delta\left(B_{0}, \ldots, B_{m}\right)$ and $g$ is some integer. Moreover the numbers

$$
\begin{aligned}
& \left\{(-1)^{i} \operatorname{det}\left(A_{0}, \ldots, \check{A}_{i}, \ldots, A_{n}, B_{0}, \ldots, B_{m}\right) ;\right. \\
& \left.\quad(-1)^{n+j} \operatorname{det}\left(A_{0}, \ldots, A_{n}, B_{0}, \ldots, \check{B}_{j}, \ldots, B_{m}\right), g\right\}
\end{aligned}
$$

are either all positive or all negative.

Proof. Set $V:=\operatorname{lin}_{\mathbf{Q}}\left\{\left[A_{i}\right],\left[B_{j}\right]: i=0, \ldots, n, j=0, \ldots, m\right\}$. The functions 


$$
\begin{aligned}
& \operatorname{det}\left(\cdot, A_{0}, \ldots, \check{A}_{i}, \ldots, \check{A}_{j}, \ldots, A_{n}, B_{0}, \ldots, B_{m}\right), \\
& \operatorname{det}\left(\cdot, A_{0}, \ldots, \check{A}_{i}, \ldots, A_{n}, B_{0}, \ldots, \check{B}_{j}, \ldots, B_{m}\right), \\
& \operatorname{det}\left(\cdot, A_{0}, \ldots, A_{n}, B_{0}, \ldots, \check{B}_{i}, \ldots, \check{B}_{j}, \ldots, B_{m}\right)
\end{aligned}
$$

define functionals on $V$ via

$$
V \ni w \longrightarrow \operatorname{det}(w, *, \ldots, *):=\operatorname{det}(w,[*], \ldots,[*]) \in \mathbf{Q} .
$$

Set

$$
\begin{aligned}
v:= & \sum(-1)^{i} \operatorname{det}\left(A_{0}, \ldots, \check{A}_{i}, \ldots, A_{n}, B_{0}, \ldots, B_{m}\right)\left[A_{i}\right] \\
& -\sum(-1)^{n+j} \operatorname{det}\left(A_{0}, \ldots, A_{n}, B_{0}, \ldots, \check{B}_{j}, \ldots, B_{m}\right)\left[B_{j}\right] .
\end{aligned}
$$

By definition $v \in V$. The set $\left\{\left[A_{i}\right],\left[B_{j}\right]: i=0, \ldots, n, j=0, \ldots, m-1\right\}$ (we omit $j=m !$ ) is a basis of $V$ (it spans $V$ because $\left[B_{m}\right]$ is a combination of the above vectors, and it is linearly independent because $\operatorname{dim} V=m+n+1)$. Hence we can write for some $a_{i}, b_{j}$

$$
v=\sum_{i=0}^{n} a_{i}\left[A_{i}\right]+\sum_{j=0}^{m-1} b_{j}\left[B_{j}\right] .
$$

Then

$$
\begin{aligned}
\operatorname{det} & \left(v, A_{0}, \ldots, \check{A}_{i}, \ldots, A_{n} ; B_{0}, \ldots, B_{m-1}\right) \\
& =a_{i} \operatorname{det}\left(A_{i}, A_{0}, \ldots, \check{A}_{i}, \ldots, A_{n}, B_{0}, \ldots, B_{m-1}\right) \\
& =(-1)^{i} a_{i} \operatorname{det}\left(A_{0}, \ldots, A_{n}, B_{0}, \ldots, B_{m-1}\right) .
\end{aligned}
$$

Thus

$$
\begin{aligned}
a_{i}=(-1)^{i} \operatorname{det}\left(v, A_{0}, \ldots, \check{A}_{i}, \ldots, A_{n}, B_{0}, \ldots, B_{m-1}\right) \\
\cdot 1 / \operatorname{det}\left(A_{0}, \ldots, A_{n}, B_{0}, \ldots, B_{m-1}\right) .
\end{aligned}
$$

Analogously

$$
\begin{aligned}
b_{j}=(-1)^{n+j} \operatorname{det}\left(v, A_{0}, \ldots, A_{n}, B_{0}, \ldots, \check{B}_{j}, \ldots, B_{m-1}\right) \\
\cdot 1 / \operatorname{det}\left(A_{0}, \ldots, A_{n}, B_{0}, \ldots, B_{m-1}\right) .
\end{aligned}
$$

By the definition of $v$,

$$
\begin{aligned}
\operatorname{det} & \left(v, A_{0}, \ldots, \check{A}_{j}, \ldots, A_{n}, B_{0}, \ldots, B_{m-1}\right) \\
& =\sum(-1)^{i} \operatorname{det}\left(A_{0}, \ldots, \check{A}_{i}, \ldots, A_{n}, B_{0}, \ldots, B_{m}\right) \\
& \cdot \operatorname{det}\left(A_{i}, A_{0}, \ldots, \check{A}_{j}, \ldots, A_{n}, B_{0}, \ldots, B_{m-1}\right) \\
& \quad-\sum(-1)^{n+i} \operatorname{det}\left(A_{0}, \ldots, A_{n}, B_{0}, \ldots, \check{B}_{i}, \ldots, B_{m}\right) \\
& \cdot \operatorname{det}\left(B_{i}, A_{0}, \ldots, \check{A}_{j}, \ldots, A_{n}, B_{0}, \ldots, B_{m-1}\right) \\
& =(-1)^{j} \operatorname{det}\left(A_{0}, \ldots, \check{A}_{j}, \ldots, A_{n}, B_{0}, \ldots, B_{m}\right)(-1)^{j} \\
& \cdot \operatorname{det}\left(A_{0}, \ldots, A_{n}, B_{0}, \ldots, B_{m-1}\right) \\
& \quad-(-1)^{n+m} \operatorname{det}\left(A_{0}, \ldots, A_{n}, B_{0}, \ldots, B_{m-1}\right)(-1)^{n+m} \\
& \cdot \operatorname{det}\left(A_{0}, \ldots, \check{A}_{j}, \ldots, A_{n}, B_{0}, \ldots, B_{m}\right)=0 .
\end{aligned}
$$


We have used the facts that $\operatorname{det}\left(A_{i}, A_{0}, \ldots \check{A}_{j}, \ldots, A_{n}, B_{0}, \ldots, B_{m-1}\right) \neq 0$ only for $i=j$ and $\operatorname{det}\left(B_{i}, A_{0}, \ldots A_{n}, B_{0}, \ldots, B_{m-1}\right) \neq 0$ only for $i=m$. Also antisymmetry and bilinearity of determinant have been applied. Analogously,

$$
\operatorname{det}\left(v, A_{0}, \ldots, A_{n}, B_{0}, \ldots, \check{B}_{j}, \ldots, B_{m-1}\right)=0 .
$$

Consequently by the above $v=0$.

To show the second part of the assertion, note that

$$
P=\sum_{i=0}^{n} t_{i} A_{i}=\sum_{j=0}^{n} s_{j} B_{j} \text { for some } t_{i}, s_{j}>0, \quad \sum t_{i}=\sum s_{j}=1 .
$$

We can treat $A_{i}, B_{j}$ as vectors in the standard basis of our lattice $\mathbf{Z}^{m}$. Then $\left[A_{i}\right]=k_{i} A_{i} \quad\left[B_{j}\right]=p_{j} B_{j}$, where $k_{i}, p_{j}>0$. Rewrite (1) as,

$$
p[P]=\sum t_{i}^{\prime}\left[A_{i}\right]=\sum s_{j}^{\prime}\left[B_{j}\right]
$$

where $t_{i}^{\prime}=t_{i} / k_{i}, s_{j}^{\prime}=s_{j} / p_{j}$, and $p$ is the inverse of the sum of the coordinates of the vector $[P]$.

Note that (2) is the only relation (up to a constant factor) between the vectors $\left[A_{i}\right],\left[B_{j}\right]$. Hence the system of numbers

$$
\begin{aligned}
\left((-1)^{n+1} \operatorname{det}\left(A_{0}, \ldots, A_{n}, B_{0}, \ldots, \check{B}_{j}, \ldots, B_{m-1}\right),\right. \\
\\
\left.\quad(-1)^{i} \operatorname{det}\left(A_{0}, \ldots, A_{i}^{v}, \ldots, A_{n}, B_{0}, \ldots, B_{m}\right), g\right)
\end{aligned}
$$

is proportional to the system of numbers $\left(t_{i}^{\prime}, s_{j}^{\prime}, p\right)$ from $(2)$. Thus we get the assertion.

11. Determinants of Simplices of the Pair $(\Delta ;\{P, Q\})$

Lemma 11.1. Let $P, Q$ belong to a simplex $\Delta=\Delta\left(A_{0}, \ldots, A_{n}\right)$. Set

$$
\begin{gathered}
\operatorname{Det} \Delta\left(A_{0}, \ldots, A_{n}\right)=s, \\
\max \left\{\operatorname{Det} \Delta\left(P, A_{0}, \ldots, \check{A}_{i}, \ldots, A_{n}\right): i=0, \ldots, n\right\}=r
\end{gathered}
$$

a) If $P, Q \in \operatorname{int} \Delta$ and $\max \left\{\operatorname{Det} \Delta\left(Q, A_{0}, \ldots, \check{A}_{i}, \ldots, A_{n}\right): i=0, \ldots, n\right\} \leq s$ then

$\max \left\{\operatorname{Det} \Delta\left(P, Q, A_{0}, \ldots, \check{A}_{i}, \ldots, \check{A}_{j}, \ldots, A_{n}\right): i=0 \ldots n, j=0, \ldots, n\right\}<r$.

b) If $\max \left\{\operatorname{Det} \Delta\left\{Q, A_{0}, \ldots, \check{A}_{i}, \ldots, A_{n}\right): i=0, \ldots, n\right\}<s$ then

$$
\max \left\{\operatorname{Det} \Delta\left(P, Q, A_{0}, \ldots, \check{A}_{i}, \ldots, \check{A}_{j}, \ldots, A_{n}\right): i=0 \ldots n, j=0, \ldots, n\right\}<r \text {. }
$$

Proof. Fix an orientation of the affine hull of the points of $\Delta$. We have representations

$$
[P]=\sum \alpha_{i}^{p}\left[A_{i}\right], \quad[Q]=\sum \alpha_{i}^{q}\left[A_{i}\right] \quad \text { for some } \quad \alpha_{i}^{p}, \alpha_{i}^{q} \geq 0
$$

Then

$$
\begin{aligned}
\operatorname{det}\left(P, A_{0}, \ldots, \check{A}_{i}, \ldots, A_{n}\right) & =\alpha_{i}^{p} \operatorname{det}\left(A_{i}, A_{0}, \ldots, \check{A}_{i}, \ldots, A_{n}\right) \\
& =(-1)^{i} \alpha_{i}^{p} \operatorname{det}\left(A_{0}, \ldots, A_{n}\right), \\
\operatorname{det}\left(Q, A_{0}, \ldots, \check{A}_{i}, \ldots, A_{n}\right) & =(-1)^{i} \alpha_{i}^{q} \operatorname{det}\left(A_{0}, \ldots, A_{n}\right), \\
\operatorname{Det}\left(Q, A_{0}, \ldots, \check{A}_{i}, \ldots, A_{n}\right) & =\alpha_{i}^{q} \operatorname{Det}\left(A_{0}, \ldots, A_{n}\right),
\end{aligned}
$$




$$
\begin{aligned}
& (* *) \\
& \operatorname{det}\left(P, Q, \ldots, A_{0}, \ldots, \check{A}_{i}, \ldots, \check{A}_{j}, \ldots, A_{n}\right) \\
& =\operatorname{det}\left(\alpha_{i}^{p}\left[A_{i}\right]+\alpha_{j}^{p}\left[A_{i}\right], \alpha_{i}^{q}\left[A_{j}\right]+\alpha_{j}^{q}\left[A_{j}\right],\left[A_{0}\right], \ldots,\left[\check{A}_{i}\right], \ldots,\left[\check{A}_{j}\right], \ldots,\left[A_{n}\right]\right) \\
& \quad=\alpha_{i}^{p} \alpha_{j}^{q} \operatorname{det}\left(A_{i}, A_{j}, A_{0}, \ldots, \check{A}_{i}, \ldots \check{A}_{j}, \ldots, A_{n}\right) \\
& \quad+\alpha_{j}^{p} \alpha_{i}^{q} \operatorname{det}\left(A_{j}, A_{i}, A_{0}, \ldots, \check{A}_{i}, \ldots, \check{A}_{j}, \ldots, A_{n}\right) \\
& \quad=\left(\alpha_{i}^{p} \alpha_{j}^{q}-\alpha_{j}^{p} \alpha_{i}^{q}\right) \operatorname{det}\left(A_{i}, A_{j}, A_{0}, \ldots, \check{A}_{i}, \ldots, \check{A}_{j}, \ldots A_{n}\right) \\
& \quad=\left(\alpha_{i}^{p} \alpha_{j}^{q}-\alpha_{j}^{p} \alpha_{i}^{q}\right)(-1)^{i+j-1} \operatorname{det}\left(A_{0}, \ldots, A_{n}\right), \\
& \operatorname{Det}\left(P, Q, \ldots, A_{0}, \ldots, \check{A}_{i}, \ldots, \check{A}_{j}, \ldots, A_{n}\right) \\
& \quad=\left|\alpha_{i}^{p} \alpha_{j}^{q}-\alpha_{j}^{p} \alpha_{i}^{q}\right| \operatorname{Det}\left(A_{0}, \ldots, A_{n}\right) .
\end{aligned}
$$

(a) By $(*))$ and the assumption on $s$ we claim that $\alpha_{i}^{q} \leq 1$ in the representation $[Q]=\sum \alpha_{i}^{q}[A]$. On the other hand, $\alpha_{i}^{p}, \alpha_{j}^{q}>0$, since $P, Q \in \operatorname{int} \Delta$. Thus,

$$
\text { Det } \begin{aligned}
& \Delta\left(P, Q, A_{0}, \ldots, \check{A}_{i}, \ldots, \check{A}_{j}, \ldots, A_{n}\right) \\
& =\left|\alpha_{i}^{p} \alpha_{j}^{q}-\alpha_{j}^{p} \alpha_{i}^{q}\right| \operatorname{Det} \Delta\left(A_{0}, \ldots, A_{n}\right) \\
& <\max \left\{\alpha_{i}^{p} \alpha_{j}^{q} \operatorname{Det} \Delta\left(A_{0}, \ldots, A_{n}\right) ; \alpha_{j}^{p} \alpha_{i}^{q} \operatorname{Det} \Delta\left(A_{0}, \ldots, A_{n}\right)\right\} \\
& \leq \max \left\{\alpha_{j}^{p} \operatorname{Det} \Delta\left(A_{0}, \ldots, A_{n}\right) ; \alpha_{i}^{p} \operatorname{Det} \Delta\left(A_{0}, \ldots, A_{n}\right)\right\} \\
& =\max \left\{\operatorname{Det} \Delta\left(P, A_{0}, \ldots, \check{A}_{j}, \ldots, A_{n}\right) ; \operatorname{Det} \Delta\left(P, A_{0}, \ldots, \check{A}_{i}, \ldots, A_{n}\right)\right\} \leq r .
\end{aligned}
$$

(b) By the assumption on $s$ and $(*)$ we have, in particular, $\alpha_{i}^{q}<1$ for all $i$. Then

$$
\begin{aligned}
\operatorname{Det} & \Delta\left(P, Q, A_{0}, \ldots, \check{A}_{i}, \ldots, \check{A}_{j}, \ldots, A_{n}\right) \\
& =\left|\alpha_{i}^{p} \alpha_{j}^{q}-\alpha_{j}^{p} \alpha_{i}^{q}\right| \operatorname{Det} \Delta\left(A_{0}, \ldots, A_{n}\right) \\
& \leq \max \left\{\alpha_{i}^{p} \alpha_{j}^{q} \operatorname{Det} \Delta\left(A_{0}, \ldots, A_{n}\right) ; \alpha_{j}^{p} \alpha_{i}^{q} \operatorname{Det} \Delta\left(A_{0}, \ldots, A_{n}\right)\right\} \\
& <\max \left\{\alpha_{j}^{p} \operatorname{Det} \Delta\left(A_{0}, \ldots, A_{n}\right) ; \alpha_{i}^{p} \operatorname{Det} \Delta\left(A_{0}, \ldots, A_{n}\right)\right\} \\
& =\max \left\{\operatorname{Det} \Delta\left(P, A_{0}, \ldots, \check{A}_{j}, \ldots, A_{n}\right) ; \operatorname{Det} \Delta\left(P, A_{0}, \ldots, \check{A}_{i}, \ldots, A_{n}\right)\right\} \leq r .
\end{aligned}
$$

Let $\mathbf{T}$ be a triangulation of an $F$-complex $\mathbf{K} . P, Q \in|T|$.

Lemma 11.2. If $P$ determines an (n)-blow-up of $\mathbf{T}$ and $Q$ determines a decreasing blow-up of $\mathbf{T}$, then $P$ determines an $(n)$-blow-up of $\mathbf{T}_{(Q)}$.

Proof. It suffices to prove the theorem for the respective blow-ups of any simplex $\Delta^{\prime} \in \mathbf{T}$ such that $P, Q \in \Delta^{\prime}$. Let $\Delta^{\prime}=\Delta\left(A_{0} \ldots, A_{l}\right)$. By assumption and Lemma $11.1 b)$,

$$
\operatorname{Det} \Delta\left(A_{0}, \ldots, \check{A}_{i}, \ldots, A_{l}, P\right) \leq n
$$

implies that

$$
\operatorname{Det} \Delta\left(A_{0}, \ldots, \check{A}_{i}, \ldots, \check{A}_{j}, \ldots, A_{l}, P, Q\right) \leq n .
$$

Finally, $\mathbf{P}$ determines an $(n)$-blow-up of $\Delta^{\prime}$ and all of $\mathbf{T}$.

Lemma 11.3. Assume that $P$ determines an $(n)$-blow-up of $\mathbf{T}$ which is not decreasing and $Q$ determines a decreasing blow-up of $\mathbf{T}$. Assume $Q \in$ int $\Delta^{\prime}$ and $P \in \Delta^{\prime}$ for some $\Delta^{\prime} \in \mathbf{T}$. Then $Q$ determines an $(n-1)$-blow-up of $\mathbf{T}_{(P)}$. 
Proof. It suffices to prove the theorem for the respective blow-ups of any $\Delta^{\prime \prime} \in \mathbf{T}$ such that $Q \in \Delta^{\prime \prime}$.

Let $\Delta^{\prime \prime}=\Delta\left(A_{0}, \ldots, A_{l}\right)$. By the assumptions we have that $P \in \Delta^{\prime \prime}$. Since $P$ determines an $(n)$-blow-up of $\Delta^{\prime \prime}$, but not a decreasing one we claim that $\operatorname{Det} \Delta^{\prime \prime} \leq$ $n$. Since $Q$ determines a decreasing blow-up of $\Delta^{\prime \prime}$, it determines $(n-1)$-blow-up of $\Delta^{\prime \prime}$. In particular

$$
\operatorname{Det} \Delta\left(A_{0}, \ldots, \check{A}_{i}, \ldots, A_{l}, Q\right) \leq n-1, \quad i=1, \ldots, n .
$$

Then by Lemma 11.1b)

$$
\operatorname{Det} \Delta\left(A_{0}, \ldots, \check{A}_{i}, \ldots, \check{A}_{j}, \ldots, A_{l}, P, Q\right) \leq n-1 .
$$

Finally, $Q$ determines an $(n-1)$-blow-up of $\Delta_{(P)}^{\prime \prime}$ and hence of the whole $\mathbf{T}_{(P)}$.

Lemma 11.4. If $\mathbf{P}$ determines an (n)-blow-up of $\mathbf{T}$ which is not decreasing and $Q$ determines a decreasing blow-up of $\mathbf{T}$, then $\mathbf{T}_{(P, Q)}$ and $\mathbf{T}_{(Q, P)}$ differ by a succession of elementary transformations of type $(n, n-1)$.

Proof. It is sufficient to prove the lemma for the respective blow-ups of any $\Delta^{\prime} \in \mathbf{T}$ such that $P, Q \in \Delta^{\prime}$.

Let $\Delta^{\prime}=\Delta\left(A_{0} \ldots, A_{l}\right)$. By arguments similar that in the previous proof we claim that Det $\Delta^{\prime} \leq n$ and $Q$ determines an $(n-1)$-blow-up of $\Delta^{\prime}$.

There are three types of simplices of the pair $\left(\Delta^{\prime} ; P, Q\right)$ :

I. $\Delta^{\prime \prime}=\Delta\left(A_{0}, \ldots, \check{A}_{i}, \ldots, A_{l}, P\right)$; then Det $\Delta^{\prime \prime} \leq n$.

II. $\Delta^{\prime \prime}=\Delta\left(A_{0}, \ldots, \check{A}_{i}, \ldots, A_{l}, Q\right)$; then Det $\Delta^{\prime \prime} \leq n-1$.

III. $\Delta^{\prime \prime}=\Delta\left(A_{0}, \ldots, \check{A}_{i}, \ldots, \check{A}_{j}, \ldots, A_{l}, P, Q\right)$; by Lemma 11.1 b) Det $\Delta^{\prime \prime} \leq$ $n-1$.

By Corollary 4.1.3 we have that each basic polytope in the elementary equivalence of $\Delta_{(P, Q)}^{\prime}$ and $\Delta_{(Q, P)}^{\prime}$ is of the form

$$
\left(\mathbf{P}^{\mathbf{B}}\right)^{\prime}=\operatorname{conv}\left(A_{i_{0}}, \ldots, A_{i_{h}}, P ; A_{i_{h+1}}, \ldots, A_{i_{g}}, Q\right)
$$

for some subset $\left\{i_{0}, \ldots, i_{g}\right\} \subset\{1, \ldots, l\}$. Thus for each elementary transformation one canonical triangulation of the corresponding basic complex $\left(\mathbf{C}^{\mathbf{B}}\right)^{\prime}$ consists of simplices of types I and III and the other one consists of simplices of types II and III. Finally, each elementary transformation is of type $(n, n-1)$.

Lemma 11.5. If $\mathbf{P}$ determines an $(n)$-blow-up of $\mathbf{T}$ and $Q$ determines a decreasing $(n)$-blow-up of $\mathbf{T}$, then $\mathbf{T}_{(P, Q)}$ and $\mathbf{T}_{(P, Q)}$ differ by elementary transformations of type $(n, n)$.

Proof. Analogously as for Lemma 11.4

Lemma 11.6. Let $n \geq 1$ and $\Delta \in \mathbf{T}$. If $\mathbf{T}$ is regular and $P \in \operatorname{int} \Delta$ determines an (n)-blow-up of $\mathbf{T}$ and $Q \in \operatorname{int} \Delta$ determines a regular blow-up of $\mathbf{T}$, then

(i) $P$ determines an $(n-1)$-blow-up of $\mathbf{T}_{(Q)}$,

(ii) $Q$ determines an $(n-1)$-blow-up of $\mathbf{T}_{(P)}$,

(iii) $\mathbf{T}_{(P, Q)}$ and $\mathbf{T}_{(Q, P)}$ differ by elementary transformations of type $(n-1, n-1)$.

Proof. Note that if $n=1$ then $P=Q$ and $\mathbf{T}_{(P)}=\mathbf{T}_{(Q)}=\mathbf{T}_{(P, Q)}=\mathbf{T}_{(Q, P)}$. Assume that $n>1$. Since $\Delta$ and $\mathbf{T}$ are regular, the multiplicative factor of $\operatorname{Star}(\Delta ; \mathbf{T})$ equals one. Since all subdivisions and elementary transformations considered in the lemma are induced by subdivisions and elementary transformations of $\Delta$, it suffices 
to prove that simplices of any triangulation of the pair $(\Delta ; P, Q)$ have determinant $\leq n-1$.

Let $\Delta=\Delta\left(A_{0}, \ldots, A_{l}\right)$. By assumption we have $[Q]=\sum_{i=0}^{l}\left[A_{i}\right]$. We can assume that $P$ determines an $(n)$-blow-up of $\mathbf{T}$ but not an $(n-1)$-blow-up. Otherwise we reduce the considerations to smaller $n$. Without loss of generality $P$ satisfies the relation

$$
[P]=n\left[A_{0}\right]+\cdots+n\left[A_{j}\right]+r_{j+1}\left[A_{j+1}\right]+\cdots+r_{l}\left[A_{l}\right]
$$

where $j \geq 0$ and $r_{s}<n$ for $j+1 \leq s \leq l$.

By the above

$$
\begin{aligned}
\Delta\left(A_{0}, \ldots \check{A}_{i}, \ldots, A_{j}, \ldots, A_{l}, P\right)=n & \text { for } i \leq j, \\
\operatorname{Det} \Delta\left(A_{0}, \ldots A_{j} \ldots, \check{A}_{i}, \ldots, A_{l}, P\right)=r_{i}<n & \text { for } i>j, \\
\operatorname{Det} \Delta\left(A_{0}, \ldots \check{A}_{i}, \ldots, A_{l}, Q\right)=1 \leq n-1 & \text { for } 0 \leq i \leq l .
\end{aligned}
$$

By Lemma 11.1 a)

$$
\text { Det } \Delta\left(A_{0}, \ldots \check{A}_{i}, \ldots, \check{A}_{j}, \ldots, A_{l}, P, Q\right)<n, \quad 0 \leq i<j \leq l .
$$

Let $\Delta^{\prime}$ be a simplex of the pair $(\Delta ; P, Q)$ such that Det $\left(\Delta^{\prime}\right)=n$. Then by the above $\Delta^{\prime}=\Delta\left(A_{0}, \ldots, \check{A}_{i_{0}}, \ldots, A_{j}, \ldots, A_{l}, P\right)$ for some $i_{0} \leq j$. From (1) we have

$$
n[Q]=[P]+\left(n-r_{j+1}\right)\left[A_{j+1}\right]+\cdots+\left(n-r_{l}\right)\left[A_{l}\right],
$$

where $\left(n-r_{j+1}\right), \ldots,\left(n-r_{l}\right)$ are positive. Thus $Q \in \operatorname{conv}\left(P, A_{j+1}, \ldots, A_{l}\right) \subset \Delta^{\prime}$. But $\Delta^{\prime}$ is a simplex of the pair $(\Delta ; P, Q)$. Hence $Q$ is a vertex of $\Delta^{\prime}$, which means $Q=P$ and $n=1$, and we get a contradiction.

\section{Main Lemma}

Lemma 11. Fix an F-complex $\mathbf{C}$ and an $n \in \mathbf{Z}_{\geq 0}$. Then the following assertions hold.

$\mathbf{A} \mathbf{1}(n)$. If $\mathbf{T}_{0}$ and $\boldsymbol{T}_{1}$ are two good triangulations of $\mathbf{C}$, that differ by an elementary transformation of type $(n, n)$ and if each triangulation $\mathbf{T}$ of $\mathbf{C}$ is good whenever $\omega^{(n+1)}(\mathbf{T}) \leq \omega^{(n+1)}\left(\mathbf{T}_{0}\right)$ or $\omega^{(n+1)}(\mathbf{T}) \leq \omega^{(n+1)}\left(\mathbf{T}_{0}\right)$, then $\boldsymbol{T}_{0}$ and $\boldsymbol{T}_{1}$ are regular equivalent.

A2 $(n)$. If $\mathbf{T}_{0}$ and $\mathbf{T}_{1}$ are two good triangulations of $\mathbf{C}$, that differ by an elementary transformation of type $(n+1, n)$ and if each triangulation $\mathbf{T}$ of $\mathbf{C}$ is good whenever $\omega^{(n+1)}(\mathbf{T}) \leq \omega^{(n+1)}\left(\mathbf{T}_{0}\right)$ or $\omega^{(n+1)}(\mathbf{T}) \leq \omega^{(n+1)}\left(\mathbf{T}_{0}\right)$, then $\mathbf{T}_{0}$ and $\mathbf{T}_{1}$ are regular equivalent.

$\mathbf{B}(n)$. If a point $P$ determines an $(n)$-blow-up of a good triangulation $\mathbf{T}$ and if each triangulation $\mathbf{T}^{\prime}$ of $\mathbf{C}$ is good whenever $\omega^{(n+2)}\left(\mathbf{T}^{\prime}\right) \leq \omega^{(n+2)}(\mathbf{T})$, then $\mathbf{T}$ is regular equivalent to $\mathbf{T}_{(P)}$.

$\mathbf{C}(n)$. If $\boldsymbol{T}$ is a triangulation all of whose simplices have determinants $\leq n+1$, then $\boldsymbol{T}$ is good.

Proof. We write $\mathbf{T}_{0} \sim \mathbf{T}_{1}$ for two good triangulations which are regular equivalent. We prove the lemma by induction on $n$.

$\mathbf{A}(0)$. For $n=0$ conditions (a) from A1 and (a) from A2 are satisfied by the empty set. Therefore the assertions are valid automatically.

$\mathbf{B}(0)$. Let $P \in \Delta$ for $\Delta \in \mathbf{T}$. If $\Delta$ is not regular then Det $\Delta>1$. Hence the (1)-blow-up at $P$ is decreasing. Thus we can apply Lemma 9.13. 
Now suppose that $\Delta$ is regular. Since $P$ determines a (1)-blow-up, then by Lemma 9.4 it determines a regular blow-up of $\Delta$. Moreover, by the definition of 1-blow-up and Proposition-Definiton 9.3 all simplices of $\operatorname{Star}(\Delta ; \mathbf{T})$ are regular. Consider a decreasing decomposition $\mathbf{T}_{\left(P_{1}, \ldots, P_{k}\right)}$ of $\mathbf{T}$. Since the decomposition does not change any regular simplices, all simplices of $\operatorname{Star}(\Delta ; \mathbf{T})$ are preserved. Hence the regular blow-up at $P$ commutes with the decreasing decomposition at the sequence of points $P_{1}, \ldots, P_{k}$, i.e. $\mathbf{T}_{(P)\left(P_{1}, \ldots, P_{k}\right)}=\mathbf{T}_{\left(P_{1}, \ldots, P_{k}, P\right)}$. The last triangulation is regular equivalent to $\mathbf{T}_{\left(P_{1}, \ldots, P_{k}\right)}$.

Hence we have found an decreasing decomposition of $\mathbf{T}$ which is regular equivalent to the decreasing decomposition of $\mathbf{T}_{(P)}$. This finishes the proof of $\mathrm{B}(0)$.

$\mathbf{C}(0)$. Every triangulation $\mathbf{T}$ all of whose simplices have determinants $\leq 1$ is a regular triangulation and hence is good.

Proof of the Inductive Step of Lemma 12. We assume that $n>1$.

Case A. It follows from the assumption and from Lemmas 9.10 and 9.11 that triangulations $\mathbf{T}$ satisfying the condition $\omega_{n+1}(\mathbf{T}) \leq \omega_{n+1}\left(\mathbf{T}_{i}\right)$ where $i=0,1$ remain good if $\mathbf{T}$ is transformed by $(n)$-blow-ups or $(n, n)$-elementary transformations. Since we consider only these kind of transformations, all the triangulations in the proof below are good.

Let $\mathbf{C}^{\mathbf{B}}$ denote the basic complex of the given elementary transformation, and let $\mathbf{S}^{\mathbf{E}}$ be its elementary subdivision. Without loss of generality we can assume in both cases A1 and A2 that

$$
\max \left\{\operatorname{Det} \Delta: \Delta \in \mathbf{T}_{0 \mid \mathbf{C}^{\mathbf{B}}}\right\} \leq \max \left\{\operatorname{Det} \Delta: \Delta \in \mathbf{T}_{1_{\mid} \mathbf{C}^{\mathbf{B}}}\right\} .
$$

Let $\mathbf{P}^{\mathbf{B}}=\operatorname{conv}\left(A_{0}, \ldots, A_{n} ; B_{0}, \ldots, B_{m}\right)$ be the basic polytope of the considered elementary transformation. Set $\mathbf{T}_{0 \mid \mathbf{P}^{\mathbf{B}}}=\mathbf{A}, \mathbf{T}_{1 \mid \mathbf{P}^{\mathbf{B}}}=\mathbf{B}$. (For the notations $\mathbf{A}, \mathbf{B}$ see section 2). Then

$$
\begin{aligned}
& M_{A}:=\max \{\operatorname{Det} \Delta: \Delta \text { is a simplex of } \mathbf{A}\}, \\
& M_{B}:=\max \{\text { Det } \Delta: \Delta \text { is a simplex of } \mathbf{B}\} .
\end{aligned}
$$

By Proposition-Definition 9.3 for any triangulation $\mathbf{T}$ of $\mathbf{P}^{\mathbf{B}}$ at any $\Delta \in \mathbf{T}_{\max }$ we have

$$
\operatorname{Det}\left(\operatorname{Star}\left(\Delta ; \mathbf{S}^{\mathbf{E}}(\mathbf{T})\right)\right)=\mu \cdot \operatorname{Det} \Delta,
$$

where $\mu=\mu\left(\operatorname{Star}\left(\mathbf{P}^{\mathbf{B}} ; \mathbf{S}^{\mathbf{E}}\right)\right)$ and $\mathbf{S}^{\mathbf{E}}(\mathbf{T})$ is the induced triangulation.

In particular for any $\Delta^{\prime} \in \mathbf{A}_{\max }$ and $\Delta^{\prime \prime} \in \mathbf{B}_{\max }$

$$
\begin{aligned}
& \operatorname{Det}\left(\operatorname{Star}\left(\Delta^{\prime} ; \mathbf{T}_{0}\right)\right)=\mu \cdot \operatorname{Det} \Delta^{\prime} \\
& \operatorname{Det}\left(\operatorname{Star}\left(\Delta^{\prime \prime} ; \mathbf{T}_{1}\right)\right)=\mu \cdot \operatorname{Det} \Delta^{\prime \prime}
\end{aligned}
$$

It follows from the above that $\mu \cdot M_{A} \leq \mu \cdot M_{B}$; hence $M_{A} \leq M_{B}$.

Let $A \in \Delta\left(A_{0} \ldots A_{n}\right)$ satisfy $[A]=\sum\left[A_{i}\right] / q_{0}$, where $q_{0}$ is the GCD of the coordinates.

Let $\Delta$ be a maximal simplex of the pair $\left(\mathbf{P}^{B} ; A\right)$. Then $\Delta$ is of the form

I.

$$
\Delta\left(A_{0}, \ldots, \check{A}_{i}, \ldots, A_{n}, B_{0}, \ldots, B_{k}\right)
$$

II.

$$
\Delta\left(A_{0}, \ldots, \check{A}_{i}, \ldots, \check{A}_{j}, \ldots, A_{n}, A, B_{0}, \ldots, B_{k}\right)
$$

III.

$$
\Delta\left(A_{0}, \ldots, \check{A}_{i}, \ldots, A_{n}, A, B_{0}, \ldots, \check{B}_{j}, \ldots, B_{k}\right)
$$


I. Let $\Delta_{\mathrm{I}}=\Delta\left(A_{0}, \ldots, \check{A}_{i}, \ldots, A_{n}, B_{0}, \ldots, B_{k}\right)$. Then

$$
\begin{aligned}
& \operatorname{det}\left(A, A_{0}, \ldots, \check{A}_{i}, \ldots, A_{n}, B_{0}, \ldots, \check{B}_{j}, \ldots, B_{k}\right) \\
& \quad=(-1)^{i} / q_{0} \cdot \operatorname{det}\left(A_{0}, \ldots, A_{n}, B_{0}, \ldots, \check{B}_{j}, \ldots, B_{k}\right) .
\end{aligned}
$$

Thus

$\operatorname{Det} \Delta_{\mathrm{I}}=\operatorname{Det} \Delta\left(A, A_{0}, \ldots, \check{A}_{i}, \ldots, A_{n}, B_{0}, \ldots, \check{B}_{j}, \ldots, B_{k}\right) \leq M_{A}$.

II. Let $\Delta_{\mathrm{II}}=\Delta\left(A_{0}, \ldots, \check{A}_{i}, \ldots, \check{A}_{j}, \ldots, A_{n}, A, B_{0}, \ldots, \ldots, B_{k}\right)$. Then

$$
\begin{aligned}
\operatorname{det}( & \left.A, A_{0}, \ldots, \check{A}_{i}, \ldots, \check{A}_{j}, \ldots, A_{n}, B_{0}, \ldots, B_{k}\right) \\
= & 1 / q_{0} \cdot \operatorname{det}\left(A_{i}, A_{0}, \ldots, \check{A}_{i}, \ldots, \check{A}_{j}, \ldots, A_{n}, B_{0}, \ldots, B_{k}\right) \\
& +1 / q_{0} \cdot \operatorname{det}\left(A_{j}, A_{0}, \ldots, \check{A}_{i}, \ldots, \check{A}_{j}, \ldots, A_{n}, B_{0}, \ldots, B_{k}\right) \\
= & (-1)^{i} / q_{0} \cdot \operatorname{det}\left(A_{0}, \ldots, \check{A}_{j}, \ldots, A_{n}, B_{0}, \ldots, B_{k}\right) \\
& \quad-(-1)^{j} / q_{0} \cdot \operatorname{det}\left(A_{0}, \ldots, \check{A}_{i}, \ldots, A_{n}, B_{0}, \ldots, B_{k}\right) \\
= & (-1)^{i+j} / q_{0} \cdot\left\{(-1)^{j} \cdot \operatorname{det}\left(A_{0}, \ldots, \check{A}_{j}, \ldots, A_{n}, B_{0}, \ldots, B_{k}\right)\right. \\
& \left.-(-1)^{i} \operatorname{det}\left(A_{0}, \ldots, \check{A}_{i}, \ldots, A_{n}, B_{0}, \ldots, B_{k}\right)\right\} .
\end{aligned}
$$

By Lemma 10 both last numbers in the braces are negative or both are positive. Thus

$$
\operatorname{Det} \Delta_{\mathrm{II}}=\operatorname{Det} \Delta\left(A, A_{0}, \ldots, \check{A}_{i}, \ldots, \check{A}_{j}, \ldots, A_{n}, B_{0}, \ldots, B_{k}\right)<M_{B} .
$$

III. Let $\Delta_{\mathrm{III}}=\Delta\left(A_{0}, \ldots, \check{A}_{i}, \ldots, A_{n}, B_{0}, \ldots, B_{k}\right)$.

Let $P:=\Delta\left(A_{0}, \ldots, A_{n}\right) \cap \Delta\left(B_{0}, \ldots, B_{k}\right)$. By Lemma 10, there exists some $p \in \mathbf{Z}$ such that

$$
p[P]=\sum(-1)^{i} \operatorname{det}\left(A_{0}, \ldots, \check{A}_{i}, \ldots, A_{n}, B_{0}, \ldots, B_{k}\right)\left[A_{i}\right] .
$$

Moreover, $p$ and $(-1)^{i} \operatorname{det}\left(A_{0}, \ldots, \check{A}_{i}, \ldots, A_{n}, B_{0}, \ldots, B_{k}\right)$ have the same sign. Without loss of generality we can assume that they are positive. Hence $p \in \mathbf{N}$ and

$$
p[P]=\sum \operatorname{Det} \Delta\left(A_{0}, \ldots, \check{A}_{i}, \ldots, A_{n}, B_{0}, \ldots, B_{k}\right)\left[A_{i}\right] .
$$

By definition

$$
\operatorname{Det} \Delta_{\mathrm{III}}=\operatorname{Det} \Delta\left(A_{0}, \ldots, \check{A}_{i}, \ldots, A_{n}, B_{0}, \ldots, B_{k}\right) \leq M_{B} \text {. }
$$

Assume that Det $\Delta_{\text {III }}=M_{B}$. By the definition of the point $A$,

$$
\begin{aligned}
q_{0} M_{B}[A] & =\sum_{j} M_{B}\left[A_{j}\right] \\
& =p[P]+\sum_{j}\left(M_{B}-\operatorname{Det} \Delta\left(A_{0}, \ldots, \check{A}_{j}, \ldots, A_{n}, B_{0}, \ldots, B_{k}\right)\right)\left[A_{j}\right] .
\end{aligned}
$$

Let $k_{j}:=\left(M_{B}-\operatorname{Det} \Delta\left(A_{0}, \ldots, \check{A}_{j}, \ldots, A_{n}, B_{0}, \ldots, B_{k}\right)\right.$. Then $k_{j} \geq 0$. Set $J=$ $\left\{j \in\{0, \ldots, n\}: k_{j}>0\right\}$. By the above,

$$
q_{0} M_{B}[A]=p[P]+\sum_{j \in J} k_{j}\left[A_{j}\right] .
$$

By definition $i \notin J$. Thus

$$
\left\{A_{j}: j \in J\right\} \subset \Delta\left(A_{0}, \ldots, \check{A}_{i}, \ldots, A_{n}, B_{0}, \ldots, B_{k}\right) .
$$


and

$$
P \in \Delta\left(B_{0}, \ldots, B_{k}\right) \subset \Delta\left(A_{0}, \ldots, \check{A}_{i}, \ldots, A_{n}, B_{0}, \ldots, B_{k}\right)
$$

By (1) $A \in \Delta^{\prime}=\operatorname{conv}\left(\left\{P, A_{j}: j \in J\right)\right\}$. From (2) and (3) we conclude that $\Delta^{\prime} \subseteq \Delta_{\mathrm{III}}=\Delta\left(A_{0}, \ldots, \check{A}_{i}, \ldots, A_{n}, B_{0}, \ldots, B_{k}\right)$. Thus $A \in \Delta_{\mathrm{III}}$, which contradicts the assumption that $\Delta_{\text {III }}$ is a simplex of $\left(\mathbf{P}^{\mathrm{B}} ;\{A\}\right)$. Finally, Det $\Delta_{\text {III }}<M_{B}$.

Case $\mathrm{A} 1(n)$. By the assumption, $M_{A} \cdot \mu \leq M_{B} \cdot \mu \leq n$. By the above considerations the point $A$ determines $\left(M_{B}\right)$-decomposition of $\mathbf{A}$ and $\mathbf{B}$. Hence by Corollary 9.5 it determines $(n)$-decomposition of $\mathbf{T}_{0}=\mathbf{S}^{E}\left(\mathbf{A}_{(A)}\right)$ and $\mathbf{T}_{1}=\mathbf{S}^{E}\left(\mathbf{B}_{(A)}\right)$. By $\mathbf{B}(n-1)$ we have $\mathbf{T}_{0} \sim \mathbf{T}_{0_{(A)}}$ and $\mathbf{T}_{1} \sim \mathbf{T}_{1_{(A)}}$.

$\mathbf{A}_{(A)}$ and $\mathbf{B}_{(A)}$ differ by elementary transformations whose basic polytopes are of the form

$$
\left(\mathbf{P}^{\mathbf{B}}\right)^{\prime}=\operatorname{conv}\left(A_{i_{1}}, \ldots, A_{i_{s}}, A ; B_{0}, \ldots, B_{k}, A_{i_{s+1}}, \ldots, A_{i_{h}}\right)
$$

where $\left\{i_{1}, \ldots, i_{h}\right\} \subset\{1, \ldots, n\}$. Thus one canonical triangulation of the corresponding basic complex of these transformations consists of maximal simplices of types I and II and another one consists of simplices of types II and III. Thus by the above these triangulations differ by elementary transformations of type $\left(M_{B}, M_{B}-1\right)$ and it follows from Corollary 9.6 that induced triangulations $\mathbf{S}^{\mathbf{E}}($. differ by elementary transformations of type $(n, n-1)$. We apply A2 $(n-1)$ and get $\mathbf{T}_{0(A)} \sim \mathbf{T}_{1(A)}$. Finally, $\mathbf{T}_{0} \sim \mathbf{T}_{1}$

Case A2 $(n)$. By the assumption and since $M_{A} \leq M_{B}$, we have that $M_{B} \cdot \mu \leq n+1$ and $M_{A} \cdot \mu \leq n$. If $M_{A}=M_{B}$ then $M_{A} \cdot \mu=M_{B} \cdot \mu \leq n$, and we are done by the previous case. Assume $M_{A} \leq M_{B}-1$. The point $A$ detemines an $\left(\left\{M_{B}-1\right\}\right)$ decomposition of $\mathbf{A}$ and $\mathbf{B}$. Hence it determines $(n)$-decomposition of $\mathbf{T}_{0}$ and $\mathbf{T}_{1}$. By $\mathbf{B}(n-1)$ we have $\mathbf{T}_{0} \sim \mathbf{T}_{0_{(A)}}$ and $\mathbf{T}_{1} \sim \mathbf{T}_{1_{(A)}}$.

The triangulations $\mathbf{A}_{(A)}$ and $\mathbf{B}_{(A)}$ differ by elementary transformations of type $\left(M_{B}-1, M_{B}-1\right)$, and it follows from Corollary 9.6 that induced triangulations of $\mathbf{S}^{\mathbf{E}}$ differ by elementary transformations of type $(n, n)$. By A1 $(n)$ we have $\mathbf{T}_{0(A)} \sim$ $\mathbf{T}_{1(A)}$, and finally $\mathbf{T}_{0} \sim \mathbf{T}_{1}$

Case $\mathbf{B}(n)$. All triangulations considered below are good by assumption and by Lemmas 9.10 and 9.11 .

We can assume that $P$ determines an $(n+1)$-blow-up which is not decreasing. Otherwise we are done by Lemma 9.13.

Assume $\mathbf{T}$ is not regular. Let $Q \in \operatorname{int} \Delta$ for $\Delta \in \mathbf{T}$ determine a decreasing blowup. If $P \notin \Delta$ then $\Delta \in \mathbf{T}_{(P)}$. $Q$ determines a decreasing blow-up of $\Delta$, and hence by Lemma 9.7 it determines a decreasing blow-up of $\mathbf{T}_{(P)}$. We get $\mathbf{T}_{(P, Q)} \sim \mathbf{T}_{(P)}$ by Lemma 9.13. If $P \in \Delta$, then by Lemma $11.3 Q$ determines an $(n)$-blow-up of $\mathbf{T}_{(P)}$, and we get $\mathbf{T}_{(P, Q)} \sim \mathbf{T}_{(P)}$ by $\mathrm{B}(n-1)$. By Lemma 11.4 and $\mathrm{A} 2(n)$ we get $\mathbf{T}_{(P, Q)} \sim \mathbf{T}_{(Q, P)}$. Finally, $\mathbf{T}_{(Q, P)} \sim \mathbf{T}_{(P)}$, and $P$ determines by Lemma 11.2 an $(n+1)$-blow-up of $\mathbf{T}_{(Q)}$. After finitely many steps we have that $\mathbf{T}_{(P)}^{\prime} \sim \mathbf{T}_{(P)}$, where $\mathbf{T}^{\prime}$ is a regular decreasing decomposition of $\mathbf{T}$ and $P$ determines an $(n+1)$-blow-up of $\mathbf{T}^{\prime}$.

By Lemma 11.6 we find $Q$ such that $Q$ determines a regular blow-up of $\mathbf{T}^{\prime}$, $Q$ determines an $(n)$-blow-up of $\mathbf{T}_{(Q)}^{\prime}, P$ determines an $(n)$-blow-up of $\mathbf{T}_{(Q)}$ and $\mathbf{T}_{(P, Q)}$ and $\mathbf{T}_{(Q, P)}$ differ by elementary transformations of type $(n, n)$. By $\mathrm{B}(n-1)$ we have $\mathbf{T}_{(P)}^{\prime} \sim \mathbf{T}_{(P, Q)}^{\prime}, \mathbf{T}_{(Q)} \sim \mathbf{T}_{(Q, P)}^{\prime}$. By A1 $(n)$ we have $\mathbf{T}_{(P, Q)}^{\prime} \sim \mathbf{T}_{(Q, P)}^{\prime}$. Finally, $\mathbf{T}_{(P)}^{\prime} \sim \mathbf{T}_{(Q)}^{\prime}$. Since $Q$ is the regular blow-up of $\mathbf{T}^{\prime}$, then $\mathbf{T}_{(Q)}^{\prime} \sim \mathbf{T}^{\prime}$. 
Since $\mathbf{T}^{\prime}$ is a regular decomposition of $\mathbf{T}$, then by Lemma $9.13 \mathbf{T}^{\prime} \sim \mathbf{T}$. Finally, $\mathbf{T}_{(P)} \sim \mathbf{T}$.

Case $\mathbf{C}(n)$. Let $\Gamma^{n+1}$ denote the set of all triangulations of the complex $\mathbf{C}$ with all simplices having determinants $\leq n+1$. Let $\Gamma_{i}^{n+1}$ denote the set of all triangulations of $\mathbf{C}$ with all simplices having determinants $\leq n+1$ and containing at most $i$ maximal simplices with determinants $=n+1$.

We show that all triangulations in $\Gamma^{n+1}$ are good. By induction on $i$ we prove that all triangulations in $\Gamma_{i}^{n+1}$ are good. Then $\bigcup_{i=0}^{\infty} \Gamma_{i}^{n+1}=\Gamma^{n+1}$ yields the assertion of $\mathrm{C}(n)$.

For $i=0$ we have $\Gamma_{0}^{n+1}=\Gamma^{n}$ and we are done by $\mathrm{C}(n-1)$. Assume that $i \geq 1$ and all triangulations in $\Gamma_{i-1}^{n+1}$ are good.

Fix $\mathbf{T} \in \Gamma_{i}^{n+1}$. Let $\Delta \in \mathbf{T}_{\max }$ and $\operatorname{Det}(\Delta)=n+1$. Let $\mathbf{T}_{\left(P_{1}, \ldots, P_{r}\right)}$ be a regular decreasing decomposition of $\mathbf{T}$ and $s$ be the smallest index such the $P_{s} \in \Delta$. Then $\mathbf{T}_{\left(P_{1}, \ldots, P_{s}\right)} \in \Gamma_{s-1}^{n+1}$ and hence is good. Hence by Lemma 9.13 we have

$$
\mathbf{T}_{\left(P_{1}, \ldots, P_{r}\right)} \sim \mathbf{T}_{\left(P_{1}, \ldots, P_{s}\right)} .
$$

We show that

$$
\mathbf{T}_{\left(P_{1}, \ldots, P_{s}\right)} \sim \mathbf{T}_{\left(P_{s}\right)} .
$$

Set $P:=P_{s}$. For $1 \leq j \leq s-1$ we have that $\mathbf{T}_{\left(P_{1}, \ldots, P_{j}, P\right)}$ is a good triangulation. Since $P$ determines a decreasing blow-up of $\Delta \in \mathbf{T}_{\left(P_{1}, \ldots, P_{j}\right)}$, by Lemma 9.7 it determines a decreasing blow-up of $\mathbf{T}_{\left(P_{1}, \ldots, P_{j}\right)}$. Since $P_{j}$ determines a decreasing blow-up of $\mathbf{T}_{\left(P_{1}, \ldots, P_{j-1}\right)}$, it determines the $(n)$-blow-up. Therefore by Lemma 11.2 $P_{j}$ determines an $(n)$-blow-up of $\mathbf{T}_{\left(P_{1}, \ldots, P_{j-1}, P\right)}$, and we get by $\mathrm{B}(n-1)$ that

$$
\mathbf{T}_{\left(P_{1}, \ldots, P_{j-1}, P\right)} \sim \mathbf{T}_{\left(P_{1}, \ldots, P_{j-1}, P, P_{j}\right)} .
$$

By Lemma 11.5, $\mathbf{T}_{\left(P_{1}, \ldots, P_{j-1}, P, P_{j}\right)}$ differs from $\mathbf{T}_{\left(P_{1} \ldots P_{j}, P\right)}$ by elementary transformations of type $(n, n)$, which gives by $\mathrm{A} 1(n)$

$$
\mathbf{T}_{\left(P_{1}, \ldots, P_{j-1}, P, P_{j}\right)} \sim \mathbf{T}_{\left(P_{1}, \ldots, P_{j}, P\right)} .
$$

By the above

which gives

$$
\mathbf{T}_{\left(P_{1}, \ldots, P_{j}, P\right)} \sim \mathbf{T}_{\left(P_{1}, \ldots, P_{j-1}, P\right)},
$$

and finally

$$
\mathbf{T}_{\left(P_{1}, \ldots, P_{s-1}, P\right)} \sim \mathbf{T}_{(P)}
$$

$$
\mathbf{T}_{\left(P_{1}, \ldots, P_{r}\right)} \sim \mathbf{T}_{(P)} .
$$

Let $\mathbf{T}_{\left(Q_{1}, \ldots, Q_{l}\right)}$ be another regular decomposition. Analogously

$$
\mathbf{T}_{\left(Q_{1}, \ldots, Q_{l}\right)} \sim \mathbf{T}_{(Q)},
$$

where $Q \in \Delta$.

Since $Q$ determines an $(n)$-blow-up of $\mathbf{T}_{(P)}$ and $\mathrm{P}$ determines an $(n)$-blow-up of $\mathbf{T}_{(Q)}$, we get by Lemma 11.2 and $\mathrm{B}(n-1)$

$$
\mathbf{T}_{(P)} \sim \mathbf{T}_{(P, Q)}, \quad \mathbf{T}_{(Q)} \sim \mathbf{T}_{(Q, P)} .
$$

By Lemma $11.5 \mathbf{T}_{(P, Q)}$ and $\mathbf{T}_{(Q, P)}$ differ by elementary transformations of type $(n, n)$. Hence by $\mathrm{A} 1(n)$ we get

$$
\mathbf{T}_{(P, Q)} \sim \mathbf{T}_{(Q, P)} .
$$

Finally

$$
\mathbf{T}_{(P)} \sim \mathbf{T}_{(Q)}
$$


Thus any regular decreasing decompositions of $\mathbf{T}$ are regular equivalent, which means that $\mathbf{T}$ is good. This completes the proof of the inductive step in the induction on $s$.

\section{Main Theorems}

Proposition 13.1. Every triangulation of a Farey complex is good. The blow-up of a triangulation is regular equivalent to the triangulation.

Proof. Every triangulation satisfies the assumption of $\mathrm{C}(n)$ for sufficiently large $n$. Hence every triangulation is good.

Every blow-up is an (n)-blow-up for sufficiently large $n$. Therefore it satisfies the conditions in $\mathrm{B}(n)$ for a suitable $n$. Thus by $\mathrm{B}(n)$ the triangulations before and after the blow-up are regular equivalent.

Corollary 13.2. Any two triangulations of an F-complex $\mathbf{C}$ are regular equivalent. In particular, any two regular triangulations are regular equivalent.

Proof. From Proposition 13.1 we conclude that triangulations which are equivalent are regular equivalent. By Proposition 7.3 we are done.

Via the correspondence between a fan $\Sigma$ and complex $\mathbf{C}=\mathbf{C}(\Sigma)$ described in Sections 8 and 9, Proposition 13.2 can be translated in the following way.

Theorem 13.3. Let $\Sigma$ be any simplicial fan in $\mathbf{Q}^{m}$. Let $\Sigma^{\prime}, \Sigma^{\prime \prime}$ be two regular decompositions of $\Sigma$. Then there exists a sequence of regular decompositions $\Sigma_{i}$ of $\Sigma, i=0, \ldots, n$, such that $\Sigma_{0}=\Sigma^{\prime}, \Sigma_{n}=\Sigma^{\prime \prime}$, and each $\Sigma_{i+1}$ is obtained from $\Sigma_{i}$ by a regular blow-up or regular blow-down.

Since for each two fans $\Sigma^{\prime}, \Sigma^{\prime \prime}$ such that $\left|\Sigma^{\prime}\right|=\left|\Sigma^{\prime \prime}\right|$ we can find a common regular decomposition $\Sigma$, then by Theorem 13.3. we prove Theorem A.

\section{REFERENCES}

[1] V.I. Danilov: The geometry of toric varieties, Russ. Math.Surveys 33(2)(1978) 97-154. MR 80g:14001

[2] V.I. Danilov: The birational geometry of toric 3-folds. Izv. Akadem. Nauk SSSR Ser. Mat 46 (1982), 971-982; English transl., Math. USSR Izv. 21 (1983), 269-280. MR 84e:14008

[3] P. Griffiths, J. Harris: Principles of algebraic geometry, A Wiley-Interscience Publication. John Wiley \& Sons, New York, Chichester, Brisbane, Toronto, 1978. MR 80b:14001

[4] Branko Grunbaum: Convex polytopes, Pure and Applied Mathematics, Volume XVI, John Wiley \& Sons, New York, 1967. MR 37:2085

[5] H. Hironaka: Resolution of singularities of an algebraic varieties over a field of characteristic xero. I, II, Annals of Math. 79 (1964), 109-203, 205-326. MR 33:7333

[6] G. Kempf, F. Knudsen, D. Mumford, B.Saint-Donat: Toroidal embeddings, Lecture Notes in Math., vol. 339, Springer-Verlag, Berlin, Heidelberg, 1973. MR 44:299

[7] T. Oda: Torus Embeddings and Applications, Tata Inst Fund. Research, Bombay, SpringerVerlag, 1978. MR 81e:14001

[8] T. Oda: Convex Bodies and Algebraic Geometry. An Introduction to the Theory of Toric Varieties, Springer-Verlag, 1988. MR 88m:14038

[9] M. Reid: Decompositions of toric morphisms, Arithmetic and Geometry, II. Progress in Math. 36 (1983), 395-418. MR 85e:14071

[10] M. Teicher: On toroidal embeddings of 3-folds, Isr. J. Math 57 (1987), 46-67. MR 88m:14008

Institute of Matematics, Warsaw University, Ul. Banacha 2, 02-097 Warszawa, POLAND

E-mail address: jwlodar@mimuw.edu.pl 